FINAL REPORT

FHWA/IN/JTRP-2004/14

\title{
CONTINUATION OF RESEARCH TO DETERMINE THE EFFECTIVENESS OF ITS
} ON BORMAN EXPRESSWAY

\author{
By \\ Dr. Robert B. Jacko, Ph.D. \\ Principal Investigator \\ Professor of Civil Engineering \\ Purdue University \\ Dr. William H. Schneider IV, Ph.D. \\ Graduate Research Assistant \\ School of Civil Engineering \\ Purdue University \\ Ahmed Soliman \\ Graduate Research Assistant \\ School of Civil Engineering \\ Purdue University \\ Dr. Barry K. Partridge, Ph.D. \\ Chief, Research Division \\ Indiana Department of Transportation \\ Joint Transportation Research Program \\ Project No. C-36-67RRR \\ File No. 9-10-69 \\ SPR-2627 \\ Conducted in Cooperation with the \\ Indiana Department of Transportation \\ and the U.S. Department of Transportation \\ Federal Highway Administration
}

The contents of this report reflect the views of the authors who are responsible for the facts and accuracy of the data presented herein. The contents do not necessarily reflect the official views or policies of the Indiana Department of Transportation and the Federal Highway Administration. This report does not constitute a standard, specification, or regulation.

Purdue University

West Lafayette, Indiana

June 2004 


\section{TECHNICAL Summary}

INDOT Research

Technology Transfer and Project Implementation Information

\section{Continuation of Research to Determine the Effectiveness of ITS on Borman Expressway}

\section{Introduction}

The Borman Expressway in Northwestern Indiana is one of the most heavily traveled freeways in the United States especially among heavy duty diesel vehicles such as semi tractor trailers. Heavy duty diesel vehicles are a major source of fine particulate matter, or PM2.5, and with the revisions in the National Ambient Air Quality Standards focusing on PM2.5, vehicles with diesel engines as well as other mobile sources will be targets for significant PM2.5 emissions reductions. Intelligent Transportation Systems (ITS) present one method of reducing emissions from all vehicles including those that are diesel powered. By smoothing out traffic flow, reducing traffic congestion, and minimizing accident response and clearance times ITS could reduce vehicle acceleration and deceleration cycles which are associated with increased pollutant emissions.

An environmental monitoring station adjacent to the Borman Expressway in Northwestern Indiana has been collecting data on PM2.5, Carbon Monoxide (CO), and meteorological parameters since September of 1999. Data was collected at a 10 second, integrated averaging interval to resolve short term events associated with the rapidly changing conditions on the Borman expressway. Limited traffic data, averaged at 5 minute intervals, became available in June of 2001. The general objective of the Borman Expressway project was to correlate the local air quality to events on the Borman and determine the overall effect of the ITS on the local air quality. An "event" on the Borman is defined as anything that impairs traffic conditions on the Expressway. Construction delays, lane restrictions, accidents, weather, and excessive demand are all capable of creating congestion and impairing the utility of the expressway.

The purpose of this phase (which lasted from April 1, 2002 to April 30, 2004) was to classify the traffic conditions along the Borman Expressway in terms of the ambient air quality levels in order to further quantify the relationship between traffic conditions and local air quality levels, hence, enhance the decision making ability using the ITS protocols.

\section{Findings}

Earlier phases of this project found that the Borman Expressway supplied on average $77 \%$ of the $\mathrm{CO}$ and $72 \%$ of the $\mathrm{PM}_{2.5}$ as determined by modeling and regression analysis of traffic and environment data (SPR-2338). More over, it has been shown that the Carbon monoxide and $\mathrm{PM}_{2.5}$ concentrations and flux can increase by a factor of 2 to 3 over pre-incident / pre - congestion conditions during accident / congestion events (SPR-2338). This shows that any means that can prevent or reduce oversaturated traffic conditions should substantially reduce the presence of pollution in the local environment.
The consequent descriptive model which characterized the impact of different Bormantraffic levels on the local ambient air quality has shown a $46 \%$ improvement in local air quality from the worst-case congestion levels along the Borman Expressway to the best-case ones. The classification tree was based on the following factors:

1. Time of Day

2. Westbound Class-Nine Average Speed

3. Westbound Occupancy

4. Eastbound Class-Nine Average Speed

5. Eastbound Occupancy 
6. Westbound Class-Nine Truck Count

7. Eastbound Class-Nine Truck Count

8. The Total Eastbound and Westbound Truck Counts
The developed descriptive model has the advantage of being based on robust measured data from the Borman Expressway, hence; provided more realistic output and consequently offers a pragmatic methodology for improving local air quality as a function of traffic considerations.

\section{Implementation}

ITS is expected to be a unique tool for accomplishing improvements in the local air quality levels near the Borman Expressway. By monitoring real time traffic conditions, the Borman ITS system will optimize the smooth flow of traffic, and consequently, the Borman ITS program should result in improvement of the local air quality. By providing information about real-time traffic conditions, faster response by the Hoosier Helpers and emergency vehicles, will thereby shorten traffic backups and subsequently prevent or reduce incidentspollution productions.
Follow up projects on the Borman Expressway will produce empirical models correlating air quality and the different traffic parameters. These models will be developed and verified based on data from the current site, plus other site locations. This model and other work associated with the follow-up phase will be incorporated into a set of tables and/or a decision matrix that will assist planners and operators in making environmentally sound decisions regarding not only the Borman expressway but other expressways as well.

\section{Contacts}

For more information:

Prof. Robert Jacko

Principal Investigator

School of Civil Engineering

Purdue University

West Lafayette IN 47907

Phone: (765) 494-2199

Fax: (765) 496-1988

E-mail: jacko@ecn.purdue.edu

\author{
Indiana Department of Transportation \\ Division of Research \\ 1205 Montgomery Street \\ P.O. Box 2279 \\ West Lafayette, IN 47906 \\ Phone: (765) 463-1521 \\ Fax: (765) 497-1665
}

\section{Purdue University}

Joint Transportation Research Program

School of Civil Engineering

West Lafayette, IN 47907-1284

Phone: (765) 494-9310

Fax: (765) 496-7996 


\begin{tabular}{|c|c|c|}
\hline $\begin{array}{l}\text { 1. Report No. } \\
\text { FHWA/IN/JTRP-2004/14 }\end{array}$ & 2. Government Accession No. & 3. Recipient's Catalog No. \\
\hline \multirow{2}{*}{\multicolumn{2}{|c|}{$\begin{array}{l}\text { 4. Title and Subtitle } \\
\text { Continuation of Research to Determine the Effectiveness of ITS on Borman } \\
\text { Expressway }\end{array}$}} & $\begin{array}{l}\text { 5. } \quad \text { Report Date } \\
\text { June } 2004\end{array}$ \\
\hline & & 6. Performing Organization Code \\
\hline \multicolumn{2}{|c|}{$\begin{array}{l}\text { 7. Author(s) } \\
\text { Robert B. Jacko, William H. Schneider, Ahmed Soliman, and Barry K. Partridge }\end{array}$} & $\begin{array}{l}\text { 8. Performing Organization Report No. } \\
\text { FHWA/IN/JTRP-2004/14 }\end{array}$ \\
\hline \multirow{2}{*}{\multicolumn{2}{|c|}{$\begin{array}{l}\text { 9. Performing Organization Name and Address } \\
\text { Joint Transportation Research Program } \\
\text { School of Civil Engineering } \\
\text { Purdue University } \\
\text { West Lafayette, IN } 47907\end{array}$}} & 10. Work Unit No. \\
\hline & & $\begin{array}{l}\text { 11. Contract or Grant No. } \\
\text { SPR-2627 }\end{array}$ \\
\hline \multirow{2}{*}{\multicolumn{2}{|c|}{$\begin{array}{l}\text { 12. Sponsoring Agency Name and Address } \\
\text { Indiana Department of Transportation } \\
\text { State Office Building } \\
100 \text { North Senate Avenue } \\
\text { Indianapolis, IN } 46204\end{array}$}} & $\begin{array}{c}\text { 13. Type of Report and Period Covered } \\
\text { Final Report }\end{array}$ \\
\hline & & 14. Sponsoring Agency Code \\
\hline
\end{tabular}

15. Supplementary Notes

Prepared in cooperation with the Indiana Department of Transportation and Federal Highway Administration.

\section{Abstract}

This study is a continuation and the final phase of the SPR-2338 project that involves monitoring the ambient air quality levels adjacent to the Borman Expressway. The purpose of this phase was to classify the traffic conditions along the Borman Expressway in terms of ambient air quality levels. The duration of the phase was from April 1, 2002 to April 30, 2004. The study has shown that, in a close proximity to the Borman Expressway, air quality levels mirror the dynamic nature of traffic along the Borman, despite being surrounded by major industrial stationary sources. Therefore, a descriptive model was developed in the form of a classification tree, which characterizes the impact of different Bormantraffic levels on the local ambient air quality. This tree contains 16 different traffic clusters and shows a 46\% improvement in local air quality from the worst-case congestion levels along the Borman Expressway to the best-case ones.

17. Key Words

ITS, Intelligent, Transportation, Systems, Environmental, Air Pollution, Database, PM2.5, CO, Fine, Particulate, Matter, Carbon, Monoxide, Borman, Expressway, Ambient Monitoring

\section{Distribution Statement}

No restrictions. This document is available to the public through the National Technical Information Service, Springfield, VA 22161

\begin{tabular}{|c|c|c|c|}
\hline $\begin{array}{c}\text { 19. Security Classif. (of this report) } \\
\text { Unclassified }\end{array}$ & $\begin{array}{c}\text { 20. Security Classif. (of this page) } \\
\text { Unclassified }\end{array}$ & 22. Price & 77 \\
\hline
\end{tabular}

Form DOT F 1700.7 (8-69) 


\section{TABLE OF CONTENTS}

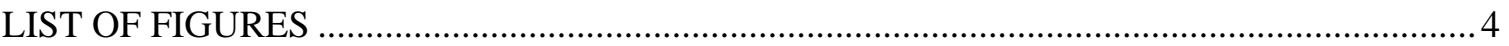

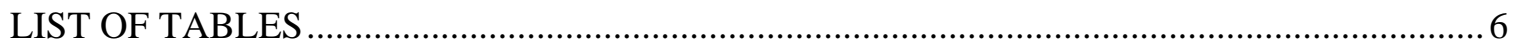

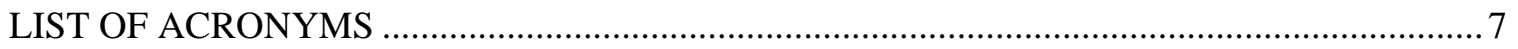

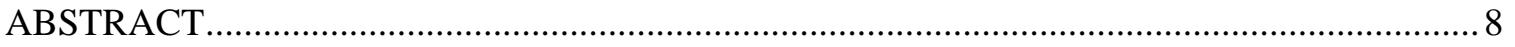

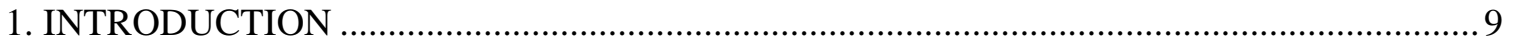

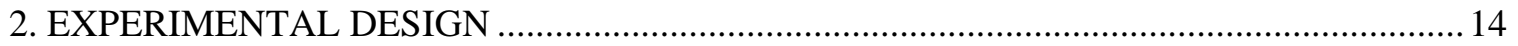

2.1. Mobile Ambient Air Quality Site location and instruments used...................................... 14

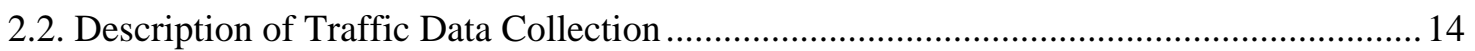

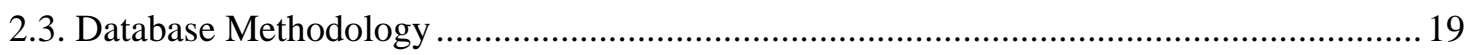

3. BORMAN EXPRESSWAY TRAFFIC AND AMBIENT LOCAL AIR QUALITY

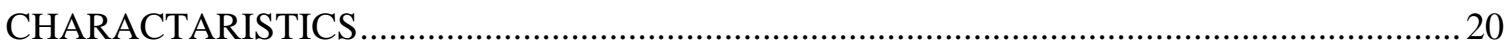

4. CLUSTER ANALYSIS STATISTICAL RESULTS AND METHODOLOGY .......................24

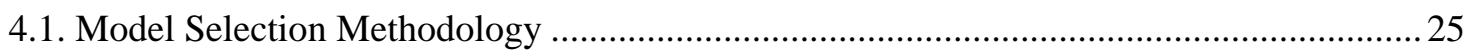

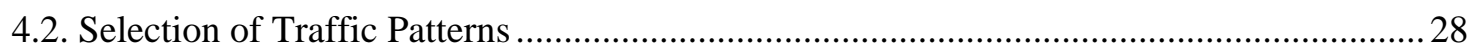

4.2.1. Total Truck Volume vs. Class-Nine Truck Volume ...................................................28

4.2.2. Total Truck Speeds vs. Class-Nine Truck Speeds ..................................................... 29

4.3. Operational Conditions Along the Borman Expressway ….............................................. 30

4.3.1. Traffic Occupancy vs. Class-Nine Truck Volumes..................................................... 30

4.3.2. Eastbound and Westbound Traffic Occupancy vs. Traffic Volume Comparisons....... 33

4.3.3. Traffic Occupancy vs. Class-Nine Average Speeds................................................... 33

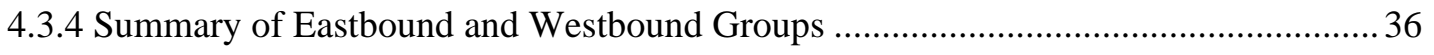

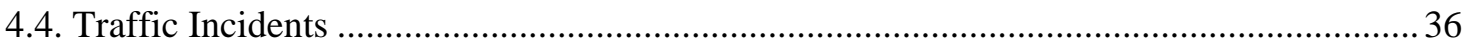

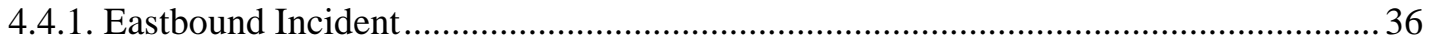

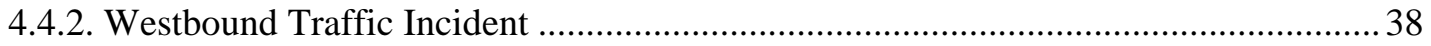

4.5. Nighttime Operational Conditions on the Borman Expressway ......................................... 39

4.5.1. Nighttime Eastbound Occupancy vs. Class-Nine Truck Volume .................................39

4.5.2. Nighttime Westbound Occupancy vs. Class-Nine Truck Volume ................................ 40

4.5.3. Nighttime Eastbound Occupancy vs. Eastbound Speed................................................ 41

4.5.4. Nighttime Westbound Occupancy vs. Westbound Speed ............................................ 43

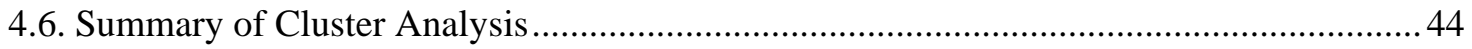




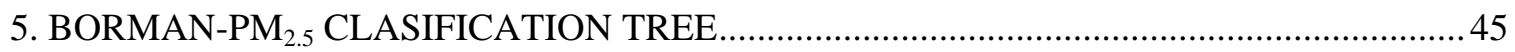

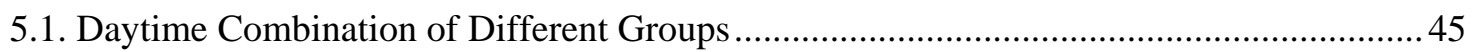

5.2. Daytime Definition of Operational Levels Along the Borman Expressway ...................... 45

5.3. Impact of Different Operational Levels on the Borman Expressway................................ 47

5.3.1. Daytime ANOVA for the Six Operational Levels .......................................................... 49

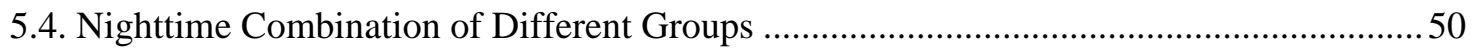

5.5. Eight Descriptive Factors for the Classification Tree......................................................... 52

5.6. The Classification Tree Model for Nighttime Conditions ................................................... 55

5.7. The Classification Tree Model for Daytime Conditions .................................................... 57

5.7.1. Classification Tree Daytime Branch One..................................................................... 57

5.7.2. Comparison Between Normal Daytime and Nighttime Clusters ................................. 60

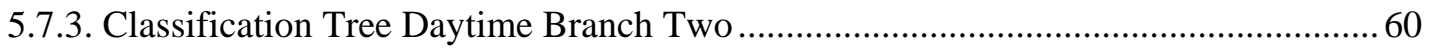

5.7.4. Classification Tree Daytime Branch Three ................................................................ 62

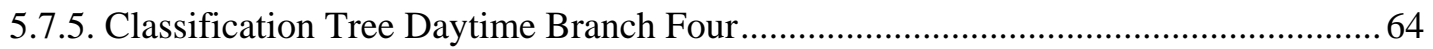

6. CLUSTER-FREQUANCY OF OCCURRENCE ON THE BORMAN EXPRESSWAY..........68

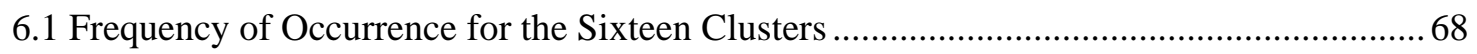

6.1.1 Daytime Frequency of Occurrence for the Twelve Clusters ........................................ 68

6.1.2 Nighttime Frequency of Occurrence for the Different Clusters ................................... 69

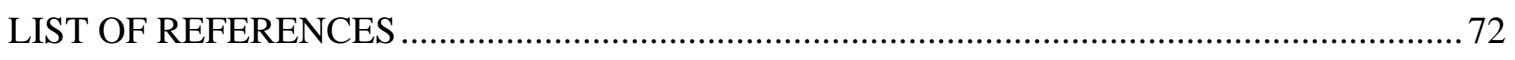

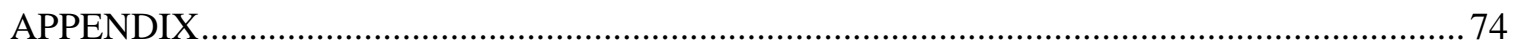




\section{LIST OF FIGURES}

Figure 2.1: Major roads and highways in Lake County, IN with respect to the PU-Hessville Site.

Figure 2.2: Kennedy Traffic Cabinet Milepost 3.5 (Similar setup as Indianapolis Cabinet)......16

Figure 2.3: Borman Expressway Weigh in Motion ...............................................................17

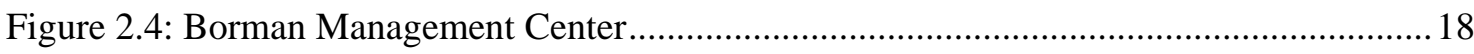

Figure 2.5: Creation of Compiled Dataset from Environmental, Indianapolis, and WIM Dataset.

Figure 3.1: May 2002 combined one week sample of PM2.5 and CO flux time series..............20

Figure 3.2: March to May 2002 combined one week sample of traffic parameters time series of west bound (W) at the Indianapolis Blvd exit.

Figure 3.3: May 2002 combined daily averages data sample of PM2.5 and CO flux time

Series.

Figure 3.4: March to May 2002 combined daily average data sample of traffic parameters time series of west bound (W) at the Indianapolis Blvd exit. 22

Figure 3.5: March to May 2002 combined daily average data sample of \% ratio of class

9-trucks to other vehicles time series of west bound at WIM station east of Site 22

Figure 3.6: The Fast Fourier Transform (FFT) spectral analysis diagram of \% occupancy data showing peaks corresponding to the daily and weekly periods. 23

Figure 4.1: Total Truck Volumes vs. Class-Nine Truck Volumes .........................................29

Figure 4.2: Total Truck Average Speed vs. Class-Nine Average Speed ................................... 30

Figure 4.3: Eastbound Occupancy vs. Class-Nine Truck Volumes ............................................31

Figure 4.4: Westbound Occupancy vs. Westbound Class-Nine Volume...................................32

Figure 4.5: Eastbound Occupancy vs. Eastbound Class-Nine Speed ..........................................34

Figure 4.6: Westbound Occupancy vs. Westbound Class-Nine Speed........................................35

Figure 4.7: Eastbound Congestion Period, April 30, 2002 ........................................................37

Figure 3.8: Westbound Congestion Period, May 28, 2002 ........................................................ 39

Figure 4.9: Nighttime Eastbound Occupancy vs. Class-Nine Truck Volumes ............................40

Figure 4.10: Nighttime Westbound Occupancy vs. Class-Nine Truck Volume .........................41 
Figure 4.11: Nighttime Eastbound Occupancy vs. Eastbound Class-Nine Truck Speed.

Figure 4.12: Nighttime Westbound Occupancy vs. Westbound Class-Nine

Truck Speed .43

Figure 5.1: Daytime Frequency of Occurrence for Different Operational Levels 48

Figure 5.2: Nighttime Frequency of Occurrence for Different Operational Levels .51

Figure 5.3: Classification Tree Model for Borman Expressway .54

Figure 5.4: Nighttime Branches of Classification Tree Model .55

Figure 5.5: $\mathrm{PM}_{2.5}$ Flux vs. Nighttime Groups .57

Figure 5.6: Classification Tree for Normal Daytime Operations. .58

Figure 5.7: Daytime Classification Tree Branch for Normal Traffic Levels .59

Figure 5.8: Daytime Classification Tree For Congestion Eastbound 61

Figure 5.9: Daytime Classification Tree for Increased Instability in the Westbound Direction. 63

Figure 5.10: Daytime Classification Tree Westbound Comparison .64

Figure 5.11: Daytime Classification Tree Westbound Congestion...........................................66

Figure 6.1: Daytime Frequency of Occurrence for the Different Clusters.................................68

Figure 6.2: Nighttime Frequency of Occurrence for Different Clusters ..................................69

Figure A.1: A sample of 5-min averaged traffic volume on Northwestern Ave, West Lafayette, IN. May 24 to May 28, 2004. .74

Figure A.2: A sample of 5-min averaged traffic occupancy on Northwestern Ave, West Lafayette, IN. May 24 to May 28, 2004. . .75

Figure A.3: A sample of 5-min averaged traffic time-mean-speed on Northwestern Ave, West Lafayette, IN. May 24 to May 28, 2004 75

Figure A.4: Estimated travel times on Northwestern Ave, West Lafayette, IN, for a lane segment of 0.5 miles. May 13, 2004, from 1:40 PM to 5:20 PM 76

Figure A.5: Estimated space-mean-speeds, and time-mean-speeds on Northwestern Ave, West Lafayette, IN, for a lane segment of 0.5 miles. May 13, 2004, from 1:40 PM to 5:20 PM ........77 


\section{LIST OF TABLES}

Table 5.1: Eastbound and Westbound Daytime Operational Levels

.46

Table 5.2: Daytime Frequency of Occurrence Between Traffic and Environmental Dataset and

Total Traffic Dataset.

Table 5.3: ANOVA Table for Daytime Correlation between Operational Levels and Ambient

$\mathrm{PM}_{2.5}$ Flux

Table 5.4. Nighttime Combination of Different Groups for Different Nighttime Operational

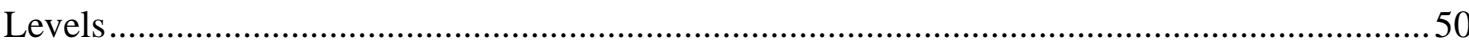

Table 5.5: Frequency of Occurrence between Nighttime Traffic and Environmental Dataset and

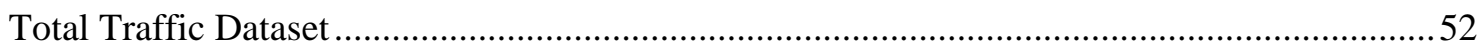

Table 5.6: ANOVA Output for the Different Cluster Conditions............................................67 


\section{LIST OF ACRONYMS}

ATIS

ATMS

CARB

DOT

DYNASMART

EMFAC

EPA

ESAL

FHWA

FTP

INDOT

ISCST3

ITS

HD

LD

NAAQS

PM

$\mathrm{PM}_{2.5}$

$\mathrm{PM}_{10}$

SQL

TEOM

WIM
Advanced Traveler Information Systems

Advanced Traffic Management Systems

Californian Air Research Board

Department of Transportation

Dynamic Network Assignment Simulation Model for Advanced Road

Telematics

Emission Factor Model

Environmental Protection Agency

Equivalent Single Axle Loading

Federal Highway Administration

Federal Testing Procedure

Indiana Department of Transportation

Industrial Source Complex Short Term Model Version 3

Intelligent Transportation Systems

Heavy-Duty Vehicles

Light-Duty Vehicles

National Ambient Air Quality Standards

Particulate Matter

Particulate Matter Less than 2.5 Microns

Particulate Matter Less than 10 Microns

Structured Query Language

Tapered Element Oscillating Microbalance

Weigh in Motion 


\section{INTRODUCTION}

The study of meteorology and air pollution of urban areas is of importance due to the harmful effects the excess air pollutants on the environment and human health. Dispersion and dilution of air pollutants in urban areas is strongly influenced by meteorological conditions as well as the terrain. Buildings and high rises contribute to the change in wind speed and direction, as well as turbulence and the thermal properties of the surface. These are due to the change in both the surface roughness and the amount of solar radiation reaching the ground in comparison to rural areas. In addition, several individual air pollution sources can be found in urban areas, ranging from motor vehicles, and small individual households or businesses, to large sources such as commercial industrial plants and power plants. Such factors contribute to the complexity of analyzing and modeling air pollution in urban areas and mega-cities (Mayer, 1999, and Mage et. al, 1991).

Air pollution regimes can be divided into three main classes based on the horizontal pollution concentration gradient (Noll et. al, 1977):

1- Micro-scale: This regime is characterized by a change in the ground level air pollution concentrations by values greater than $20 \%$ over a horizontal distance less than $100 \mathrm{~m}$. In other words, a major change in ground level concentration over a small distance. This usually occurs very near to the sources of air pollution, for example, high-traffic expressways, and large industrial sources.

2- Meso-scale: This regime is characterized by a change in the ground level air pollution concentrations by values less than $20 \%$ over a horizontal distance ranging from $100 \mathrm{~m}$ to $10,000 \mathrm{~m}$. This regime represents a community size air mass with fairly homogeneous ground level air pollution concentration, such as local background for urban areas. For example, local streets, residential and commercial space heating furnaces, and small industrial sources. Industrial and mobile sources, such as motor vehicles, contribute to the meso-scale air pollution that contaminates the ambient air in urban areas.

3- Macro-scale: This regime is characterized by a change in the ground level air pollution concentrations by values less than $20 \%$ over a horizontal distance greater than $10,000 \mathrm{~m}$. This

regime represents a regional background, which is characterized by, fairly homogeneous air pollution concentration over long distances, ranging from tens to hundreds of kilometers. 
There are different types of air pollutants that have significant impact on human health and the environment, such as carbon monoxide (CO), hazardous air pollutants (HAPs), lead (Pb), nitrogen oxides $\left(\mathrm{NO}_{\mathrm{x}}\right)$, particulate matter $\left(\mathrm{PM}_{10}\right.$, and $\left.\mathrm{PM}_{2.5}\right)$ and sulfur oxides $\left(\mathrm{SO}_{\mathrm{x}}\right)$. This review will focus mainly on carbon monoxide and particulate matter.

Carbon monoxide (CO) is a colorless, odorless gas that is harmful and lethal to humans at concentrations exceeding 5,000 ppm (parts per million) within a few minutes of exposure (Davis et. al 1998). A study relating the ambient carbon monoxide levels and the daily mortality in Toronto, Canada has shown that $4.7 \%$ of non-accidental deaths could be attributable to the exposure to CO (Burnett et. al, 1998). The exposure to CO decreases the ability of the blood to carry oxygen to the tissues and therefore can cause dizziness, weakness, unconsciousness, and in the extreme cases, death (Coombs, 1998). The National Ambient Air Quality Primary Standard (set to protect human health) for CO is $10,000 \mu \mathrm{g} / \mathrm{m}^{3}$ (9 ppm) for an average time of 8 hours and $40,000 \mu \mathrm{g} / \mathrm{m}^{3}$ (35 ppm) for an average time of 1 hour (Wark et. al, 1998).

Carbon monoxide is formed due to incomplete oxidation of carbon. The two main sources of atmospheric CO gas are natural sources and anthropogenic sources (sources associated with activities of human beings). An example of the natural sources is the anaerobic decomposition of carbonaceous material by soil micro-organisms. This decomposition process releases approximately $9 \times 10^{15}$ moles of methane $\left(\mathrm{CH}_{4}\right)$ to the atmosphere each year, worldwide (Seinfeld, 1975). The oxidization of $\mathrm{CH}_{4}$ forms the $\mathrm{CO}$ gas according to the following simplified process (Wofsy et. al, 1972):

$$
\begin{aligned}
& \mathrm{CH}_{4}+\mathrm{OH} \rightarrow \mathrm{CH}_{3}+\mathrm{H}_{2} \mathrm{O} \\
& \mathrm{CH}_{3}+\mathrm{O}_{2}+2(\mathrm{hv}) \rightarrow \mathrm{CO}+\mathrm{H}_{2}+\mathrm{OH}
\end{aligned}
$$

where $h v$ is the photon energy of sunlight ( $h$ is Planck's constant $=6.626 \times 10^{-34} \mathrm{Js}$ and $v$ is the frequency of light). Anthropogenic sources include motor vehicles, fossil fuel burnt in power plants to produce electricity or heat as well as industrial processes. These sources release approximately $1 \times 10^{13}$ moles of CO to the atmosphere each year, where the motor vehicles account for more than $60 \%$ of the total emission (Davis et. al, 1998). In the United Kingdom, the contribution of road vehicles has reached $90 \%$ of the total CO emissions (Chquetto et. al, 1995).

Due to these high emission percentages of $\mathrm{CO}$ from motor vehicles, several studies have been conducted to model the concentration distribution of vehicular pollutants in urban areas as well as to study the effects of exposure to such pollutants on human health. Some of these studies related the driver exposure to the different air pollutants such as Volatile Organic Compounds (VOC's) and CO to the different driving conditions (Chan et. al, 1991). 
Vehicle-CO formation is due to the incomplete oxidation of the hydrocarbons (fuel) to form $\mathrm{CO}_{2}$. The fuel oxidation rate depends on the available oxygen concentrations, the temperature of the gases, and the time left for the reaction to take place (which depends on the engine speed) (Degobert, 1995). In a rich-mixture (excess of fuel with respect to air, i.e. fuel/air ratio $>1$ ) ; the CO concentration increases with the fuel/air ratio since the lack of oxygen causes the incomplete oxidation. In a lean-mixture (excess of air with respect to fuel, i.e. fuel/air ratio $<1)$; the CO concentrations are low, and vary slightly with the fuel/air ratio. Diesel engines always run on an overall lean mixture, therefore, CO emissions are much lower than the ones produced by the gasoline engines. However, $\mathrm{CO}$ emissions from a diesel engine can increase at low loads or at a maximum loads at a high speed, this is due to the heterogeneity of the mixture, i.e. in the case of oxygen deficiencies, temperature levels or residence times that are not sufficient for the complete oxidation (to for $\mathrm{CO}_{2}$ ) to take place (Degobert, 1995).

Particulate matter such as sea salt, soil dust, smoke, sulfates, nitrates, some organic compounds, and exhaust of diesel engines, in general, are particles with sizes ranging from 0.001 to $500 \mu \mathrm{m}$ in diameter (Wark et.. al, 1998). The bulk particulate size distribution in the atmosphere ranges from 0.1 to $10 \mu \mathrm{m}$. Particles with diameters ranging from 1 to $20 \mu \mathrm{m}$ have settling velocities, yet these velocities are considerably small compared to the wind velocities and therefore these particles tend to follow the motion of the gas (air) in which they are born (Wark et.. al, 1998). Settling velocities are basically the constant-terminal velocities of these particles in the direction parallel to the earth's gravitational field. The terminal velocities are reached after the weight of the particles reach equilibrium with the forces caused by the buoyancy and the frictional drag. The average settling velocities of the particulate matter with sizes between 1 and $10 \mu \mathrm{m}$ in diameter range from $4 \times 10^{-5}$ to $300 \times 10^{-5} \mathrm{~m} / \mathrm{s}$ (Wark et.. al, 1998) (the average wind speeds observed in this study ranged from 0.5 to $4.5 \mathrm{~m} / \mathrm{s}$ ).

With the increase in both the number of vehicles on the roads and the total number of motorists traveling on highways, there has been a mounting effort to quantify the air quality near major urban freeways. Over the last two decades, the United States Environmental Protection Agency (EPA), the California Air Research Board (CARB), Academia, the local and Federal Highway Administration (FHWA), and many international organizations have focused on quantifying the impact of traffic emissions on local air quality. This project studies the Borman Expressway's effect on local air quality, and, more specifically, the effects of intelligent transportation systems (ITS) on local air quality. The Borman Expressway, interstate 80/94, is a 
sixteen-mile section of highway located in Northwest Indiana that travels in an east to west direction from the Illinois-Indiana border to interstate 90.

With a higher proportion of commercial traffic, the fleet mix along the Borman Expressway is different from the national average, according to the Federal Highway Administration. A weigh in motion station located on the Borman Expressway at milepost 5.9 recorded the approximate fleet mixes to be $49 \%$ light-duty gasoline vehicles (cars), $20 \%$ lightduty gasoline trucks, and 30\% heavy-duty diesel vehicles. The national average distribution for highways is $61 \%$ light-duty gasoline vehicles, 35\% light-duty gasoline trucks, and 3\% heavy heavy-duty diesel vehicles for a duration between January 2002 through June 2002.

The 27\% difference between Federal and Borman Expressway averages in fleet mixes indicates the importance of the expressway for commercial traffic. The Borman Expressway is the main artery for commercial traffic across the northern section of Indiana and leads into the city of Chicago as well as the steel industry near Lake Michigan. The large commercial volume decreases the operating capacity for the Expressway, which consequently adds stress to the Expressway, leads to more unstable traffic flow and the possibility of congestion with traffic occupancies higher than $18 \%$. The problem of congestion on expressways is not limited to the Borman Expressway; the Texas Transportation Institute estimated that the 50 largest urbanized areas in the United States lost more than 48 billion dollars as a result of traffic congestion in 1993 (Birst et al., 2000). In 1997 it was estimated that the 68 largest cities had more than 4.3 billion hours of delays (Lomax et al., 1999). More than $60 \%$ of all traffic incidents or congestion are a result of debris, accidents, lane closures, and maintenance, which lower the capacity of a freeway (Birst et al., 2000). Because of the high composition of commercial traffic in the Borman Expressway fleet mix, intelligent transportation systems (ITS) are designed and continue to be implemented along the Expressway to increase the efficiency and to help maintain a smoothly flowing Expressway.

These intelligent transportation systems include the Hoosier Helper program, which is designed to help distressed vehicles along the side of the expressway, clear debris, monitor traffic, provide support, and, most importantly, keep traffic flowing on the Borman Expressway. In a study by Latoski, the benefit-to-cost ratio for the Hoosier Helper program in 1996 was 4.71 to 1 for a daytime program and 13.28 to 1 for a 24-hour program operation (Latoski et al., 1999). ITS also relies on traffic monitoring systems such as inductive loop detectors near every on-ramp, and the midpoints between intersections, video detectors, message boards and weigh in motion (WIM) stations. These traffic monitors are installed to provide immediate information to state 
employees who maintain the Expressway and clear traffic occurrences. ITS also alerts motorists to traffic congestion and alternative routes that may be used to avoid congestion.

The unique blend of traffic characteristics on the Borman Expressway, such as fleet mixes and traffic volumes along with new ITS technologies, provide a distinct environment to monitor different states of traffic flow from free flow conditions to total breakdown heavy congestion periods. In addition to long-term traffic monitoring, normal operating conditions as well as predictable unstable periods will be quantified. Traffic collection from the inductive loops began on June 7, 2001 and has been sampling at five-minute time intervals. The WIM data became available to this project on January 15, 2002 and, unlike the inductive loop measurements the WIM data records all trucks real time, with no averaging intervals. In addition to traffic records, the careful placement of an ambient air monitoring station provides a better picture of the relationship between traffic parameters and local ambient air quality. Joint Transportation Research Program SPR-2338 and the continuation SPR-2627 began monitoring ambient air quality in September 1999 to the present, at a ten-second time interval. 


\section{EXPERIMENTAL DESIGN}

\subsection{Mobile Ambient Air Quality Site location and instruments used}

Figure 2.1 shows a map of the major roads and highways in Lake County, IN with respect to the mobile ambient air monitoring station location, commonly called the PU-Hessville Site. The PU-Hessville Site is approximately located at the 4.1-mile marker on the Borman Expressway. The $\mathbf{X}$ marks on the map indicate the portion used for modeling the Borman Expressway (approximately on the IL/IN border from the west to the 10.1-mile marker at the east) (SPR 2338, JTRP report). The meteorological data collected by the station included ambient temperature, wind direction, and wind speed since 1999. The meteorological instrument used at the PU-Hessville Site is a Met-One-Meteorological instrument set.

Carbon monoxide concentrations at the PU-Hessville Site are measured using a ThermoEnvironmental Instruments Model 48C, while $\mathrm{PM}_{2.5}$ concentrations are measured using a Rupperecht \& Patashnick Tapered Element Oscillating Microbalance (TEOM). Concentration data are measured every 10 (ten) seconds to be able to monitor the dynamic behavior of the pollutants caused by mobile sources on the Borman Expressway. Some hourly averaged data points are averages of approximately 43,000 (forty three thousand) measured data points. With such a large number of measured data points in the averaged values, it is expected that the measured average values represent the true (total) population with minimal error.

More details on the site locations and modeling data findings are found in SPR-2338 JTRP report.

\subsection{Description of Traffic Data Collection}

With the assistance of INDOT, traffic data collection from the Borman Expressway started on June 7, 2001 at 12:00 PM. 3M Intelligent Transportation Systems Canoga C800IS software is used to collect traffic data. The first loop detector is located near the Kennedy interchange, approximately 0.5 miles east of the air sampler at milepost 3.5. These loop detectors, which measures the lead and lag traffic occupancies and volumes from the traffic ramps and all three lanes traveling eastbound along the expressway. Westbound flow is not measured because no operational inductive loops were in service. The second loop detector 
started on November 2, 2001 at 1:00 PM. This loop detector is located at the Indianapolis Boulevard intersection, milepost 2.6.

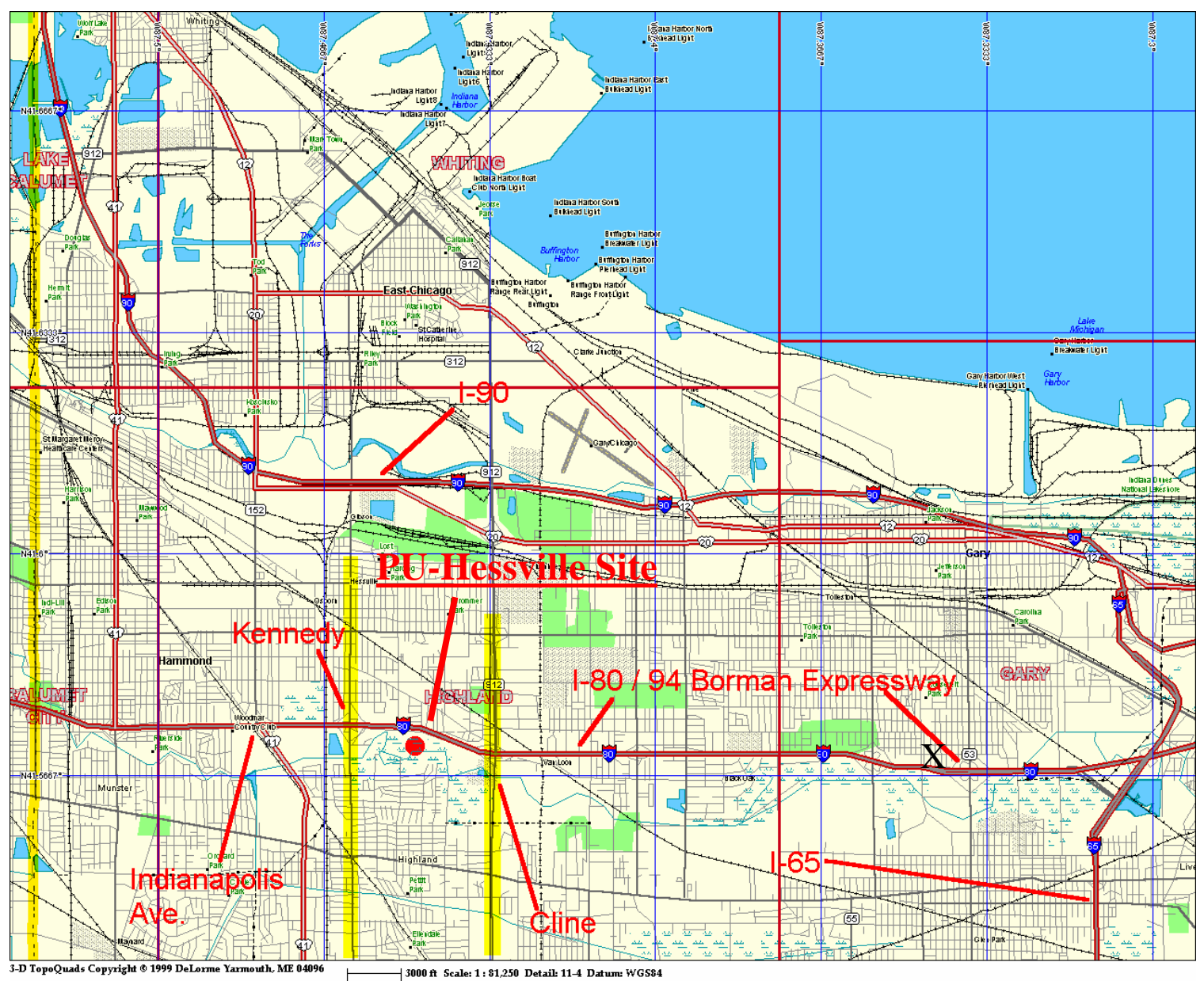

Figure 2.1: Major roads and highways in Lake County, IN with respect to the PU-Hessville Site.

Unlike the Kennedy interchange, the Indianapolis interchange collects lead and lag traffic occupancies and volumes from the ramps as well as from the three lanes in both the eastbound and westbound directions. Although the Indianapolis traffic location is rather remote from the air-monitoring site, it is the closest working eastbound/westbound loop detector. Figure 2.2 is a close up view of the traffic data collection equipment cabinet. Communication between the detector cards (A), and Purdue University is established by using Streetcom THC-3m6 cables (B) that connects to the Streetcom Trans Hub Th1 (C), from the Trans Hub Th1, a Streetcom THCPC9 cable (D) connects to a null modem (E), which is in turn connects to an external modem and then to a cellular bag phone. 
The traffic data collection system is the same for the Indianapolis and Kennedy traffic sites. Upon completion of the installation, traffic binning or time interval size became critical. One concern with the time intervals is the frequency for downloading data from the $3 \mathrm{M}$ traffic cards.

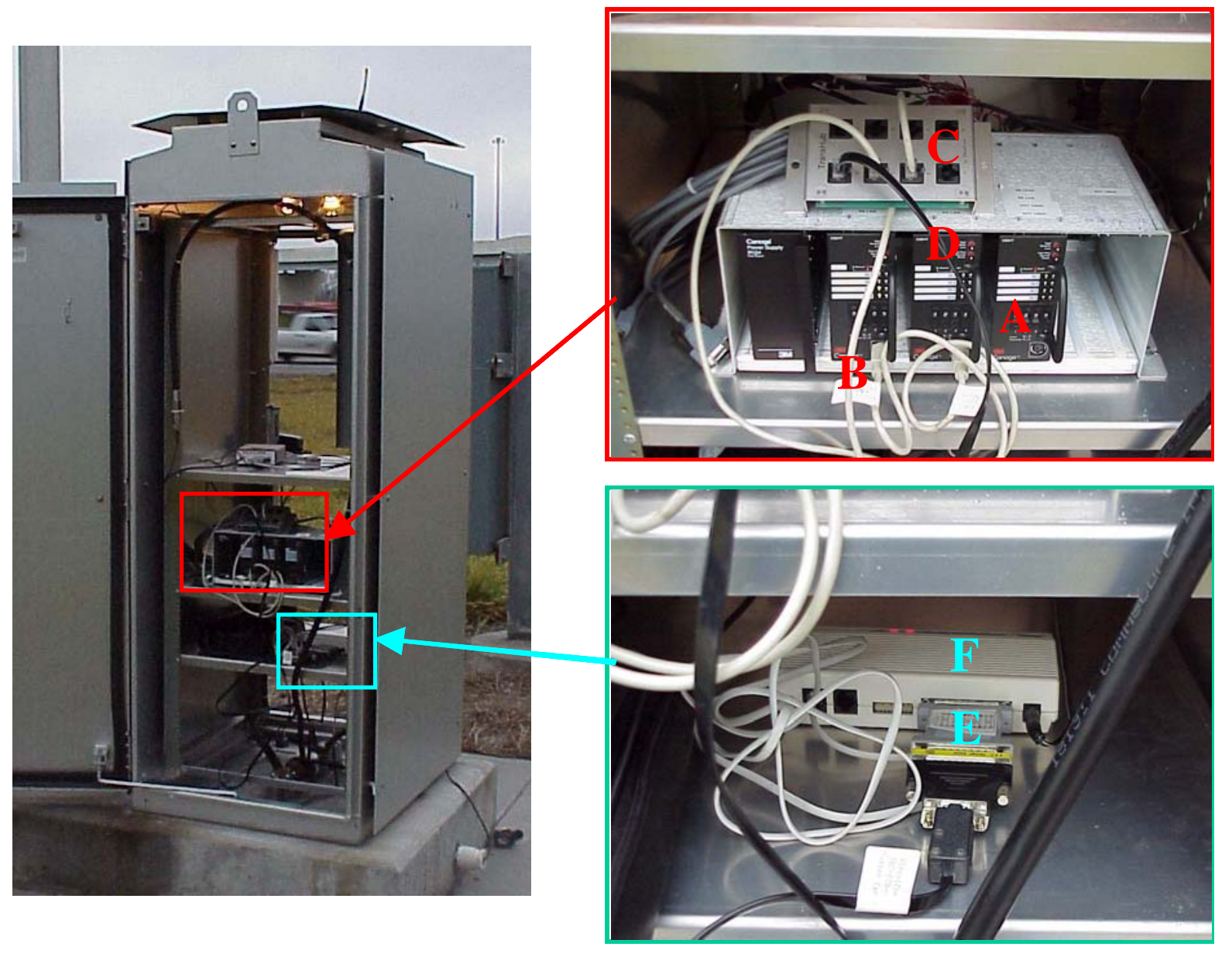

Figure 2.2: Kennedy Traffic Cabinet Milepost 3.5 (Similar setup as Indianapolis Cabinet)

After some experimentation with the traffic collection system, a five-minute sampling interval is selected, and at this size, the $3 \mathrm{M}$ traffic card could sufficiently store four to five days worth of vehicle counts.

The weigh in motion (WIM) sensors and equipment are located at milepost 5.9 on the Borman Expressway. The WIM provided real-time records of different truck parameters, ranging from the class of truck, speed, equivalent single axle load (ESAL), and total gross vehicle weights (GVW). This data, in conjunction with the inductive loops at the Indianapolis interchange, 
provided extensive traffic data. Figure 2.3 is a photograph of the eastbound WIM station located on the Borman Expressway.

There are four different components per traffic lane for the WIM. "A" represents the inductive loops that trigger the WIM system. When the inductive loops are "on" the system is ready to classify the vehicle. There are two different inductive loops, one located at the beginning and one at the end of the WIM location. " $\mathrm{B}$ " is the weigh cell, where the gross vehicle weight and the ESAL are recorded. "C" represents the piezo, where the axle spacing and the speed of the vehicles are calibrated. With the speed of the vehicle known and the axle spacing calculated, the classification of the vehicle is determined.

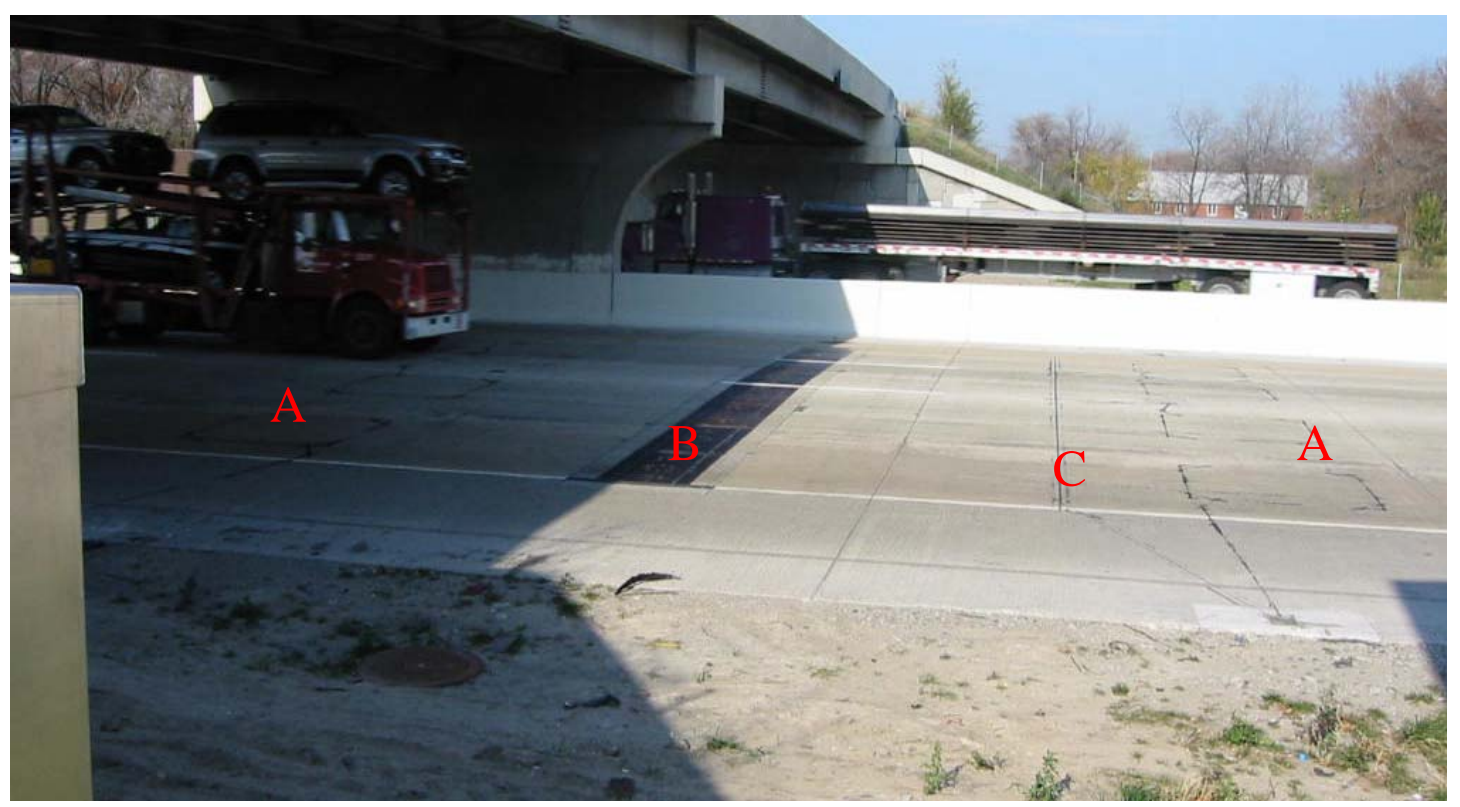

Figure 2.9: Borman Expressway Weigh in Motion

The speed of the vehicle is determined by the inductive loops " $\mathrm{B}$ " and " $\mathrm{C}$ ". The distance between the weigh cell and piezo, so the known distance divided by the time difference between the two inductive loops calculates the speed. The data from the WIM is collected and stored at the nearby traffic cabinet. The Indiana Department of Transportation (INDOT) then provides Purdue University a CD with the traffic collection recorded at the WIM station. The traffic data collection is located at two different sites, the Indianapolis inductive loops located east of the ambient monitoring station and the WIM located west of the monitoring station. The combination of the two data collection sites provided an overall view of the level of traffic 
conditions associated with the Borman Expressway, located directly in front of the ambient sampling laboratory.

The Borman Management Center is not directly involved with the collection of traffic data for this project, but from an ITS perspective it is important to discuss. As seen in Figure 2.4, the Borman Management Center has the ability to monitor the traffic on the entire Borman Expressway, and traffic incidents are quickly confirmed, and the appropriate emergency response is decided, especially whether local police, ambulances, and fire departments are required. The Borman Management Center also has the ability to contact the Hoosier Helpers, change and monitor the variable message boards, and transmit messages to the AM radio band.

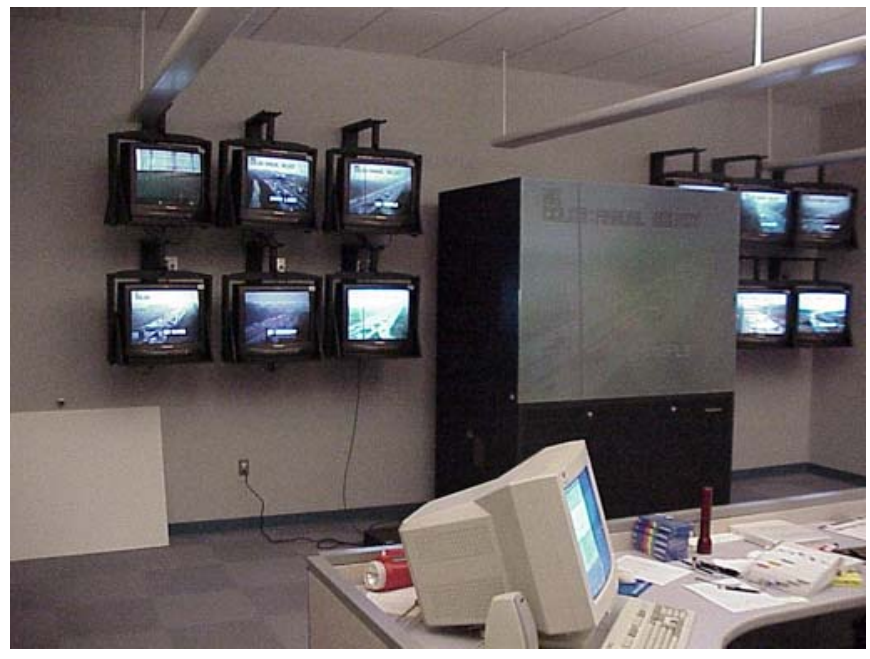

Figure 2.4: Borman Management Center

The ability to monitor traffic information, along with being able to communicate with the public enables the Borman Management Center to not only monitor the Expressway but also provide management strategies with the purpose of lowering congestion and creating a more efficient travel route.

The synthesis of the traffic data in conjunction with the environmental data will provide a dataset able to elucidate many different traffic and environmental conditions, providing some insight into the relationship between different traffic conditions and local air quality with the overall goal of improving ambient air quality downwind of the Borman Expressway. 


\subsection{Database Methodology}

Data is collected from three different locations: 1) the environmental station records all of the environmental parameters, including $\mathrm{CO}, \mathrm{PM}_{2.5}$, wind speed, wind direction, atmospheric temperature, van temperature, relative humidity, cumulative rainfall, and time of day; 2) the Indianapolis loop detectors at milepost 2.6 records average occupancies and total vehicle volumes over a five-minute average interval; and 3) a WIM station located at milepost 5.9 collects data in the eastbound and westbound directions, such as truck weights, lengths, counts, speeds, classifications, and time. In a given month, approximately 250,000 environmental records, 8,600 five-minute loop samples, and 600,000 truck records were collected and stored in the database. Because of the large volume of information that was collected, a protocol was developed to ensure the integrity of the database.

Once the three individual tables are cleaned and correctly grouped into five-minute averages, the tables are merged together. A primary key is a unique value, such as a date/time stamp, that is used by the database to merge the environmental, loop, and WIM dataset in three different tables together. In this dataset a date/time value is created that allows all three tables to be merged into two tables. One table (Environmental and Traffic Dataset) contained wind directions from 270 to 110 degrees. The second table (Traffic Dataset) is independent of wind directions. After the final tables are compiled, the two finished tables are randomly compared to the original raw datasets to guarantee the highest integrity for the final table.

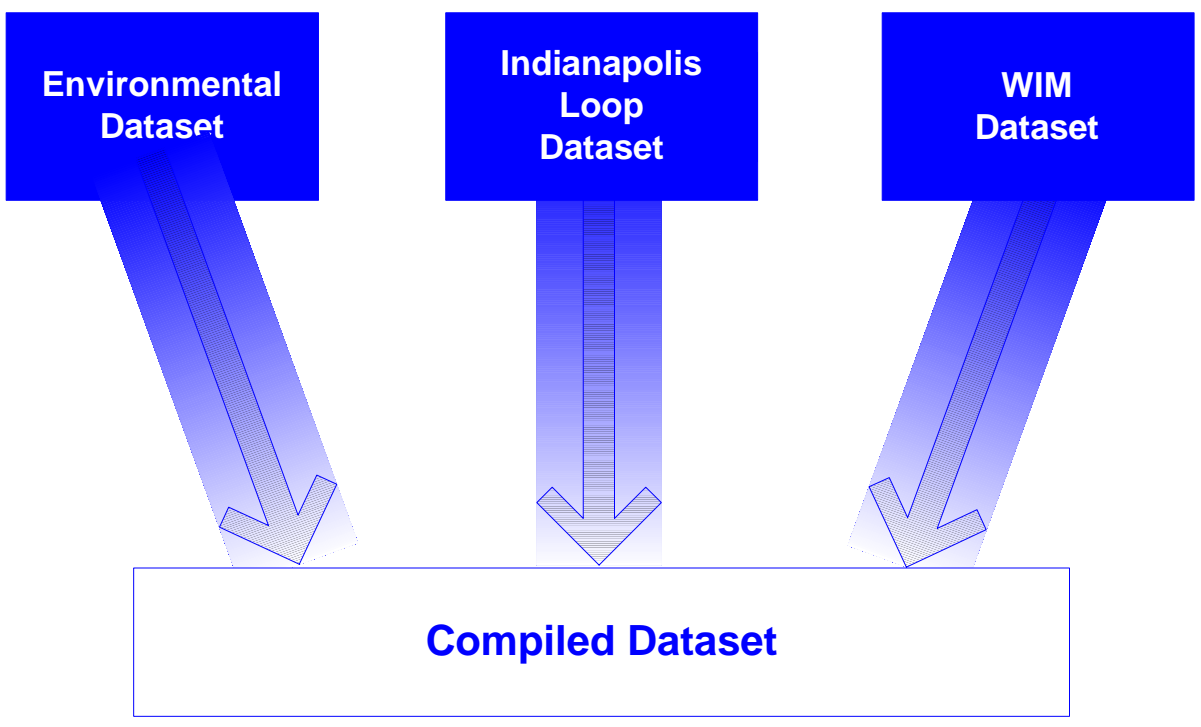

Figure 2.5: Creation of Compiled Dataset from Environmental, Indianapolis, and WIM Dataset 


\section{BORMAN EXPRESSWAY TRAFFIC AND AMBIENT LOCAL AIR QUALITY CHARACTARISTICS}

The robust environmental database has allowed the time series display of both traffic and environmental data for visual inspection as well as to be possibly used for future time series analysis (TSA). Figure 3.1 shows a sample of the environmental data that maybe used in the TSA $\left(\mathrm{PM}_{2.5}\right.$-Flux $\left[\mu \mathrm{g} / \mathrm{m}^{3} . \mathrm{m} / \mathrm{s}\right]$, and CO-Flux [ppm.m/s], respectively).

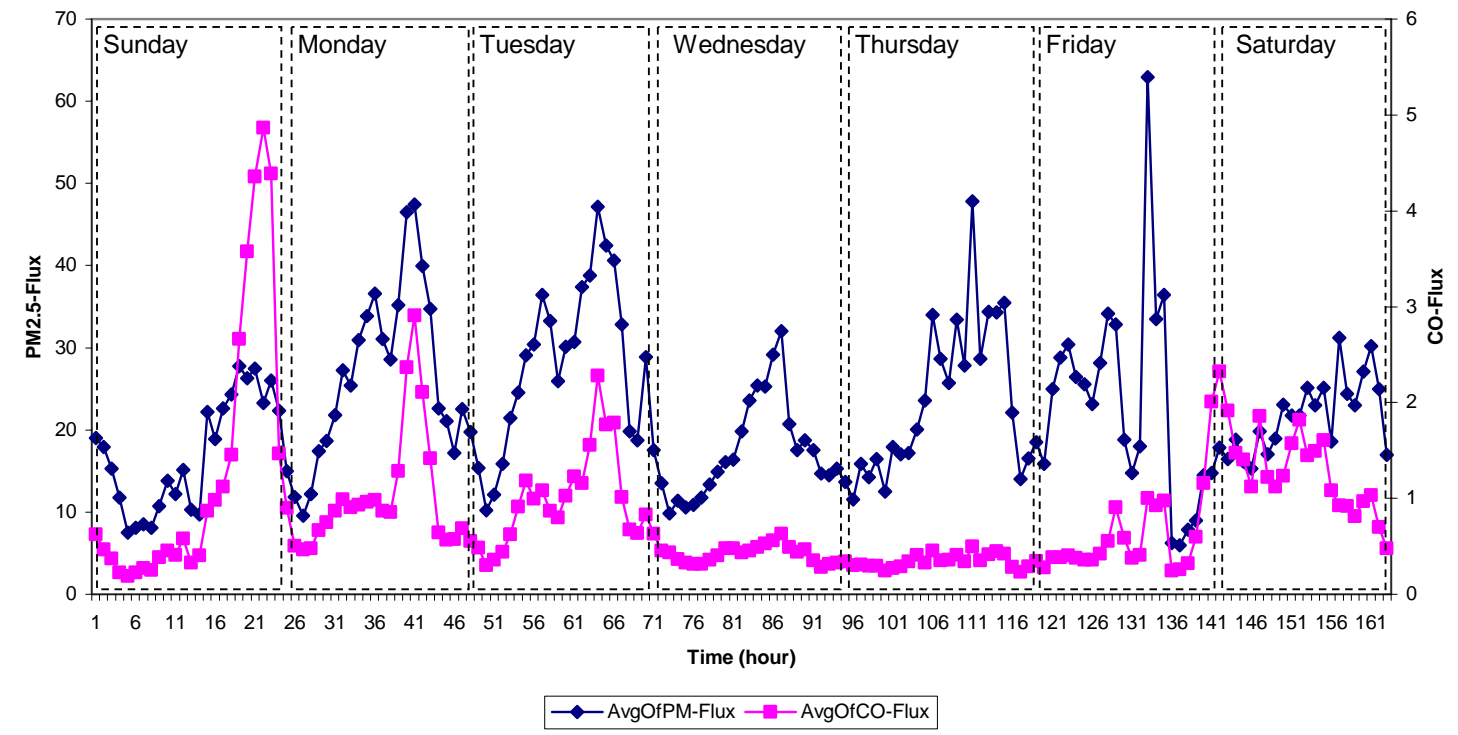

Figure 3.1: May 2002 combined one week sample of PM2.5 and CO flux time series.

Similarly, figure 3.2 shows a sample of the traffic data that may be used in the TSA (Flow [veh/h], Speed [mi/h], and Occupancy [\%], respectively).

As shown in figures 3.1 and 3.2, the measured data shows apparent daily periodicity. Data appear to change according to the day of the week, which would give an indication to weekly periodicity as well. Figures 3.3 and 3.4 shows the daily averages of the measured parameters (traffic and environmental parameters) as a function of the day of the week (1 is Sunday, 2 is Monday...etc). Seasonal periodicity is also expected in the data, which can be calculated using the monthly averages as a function of month. The drop in the CO-Flux values during the middle of the week (on Wednesday, and Thursday) in figure 3.3 is probably due to the increase of the diesel-powered class 9 trucks ratio with respect to the gasoline-powered vehicles. 
Figure 3.5 shows the \% ratio of class 9 trucks to the other types of vehicles traveling on the Borman Expressway. As shown in figure 3.5, the \% ratio goes up to 68\% during Wednesday and Thursday compared to lower values over the weekend (around 20\%).

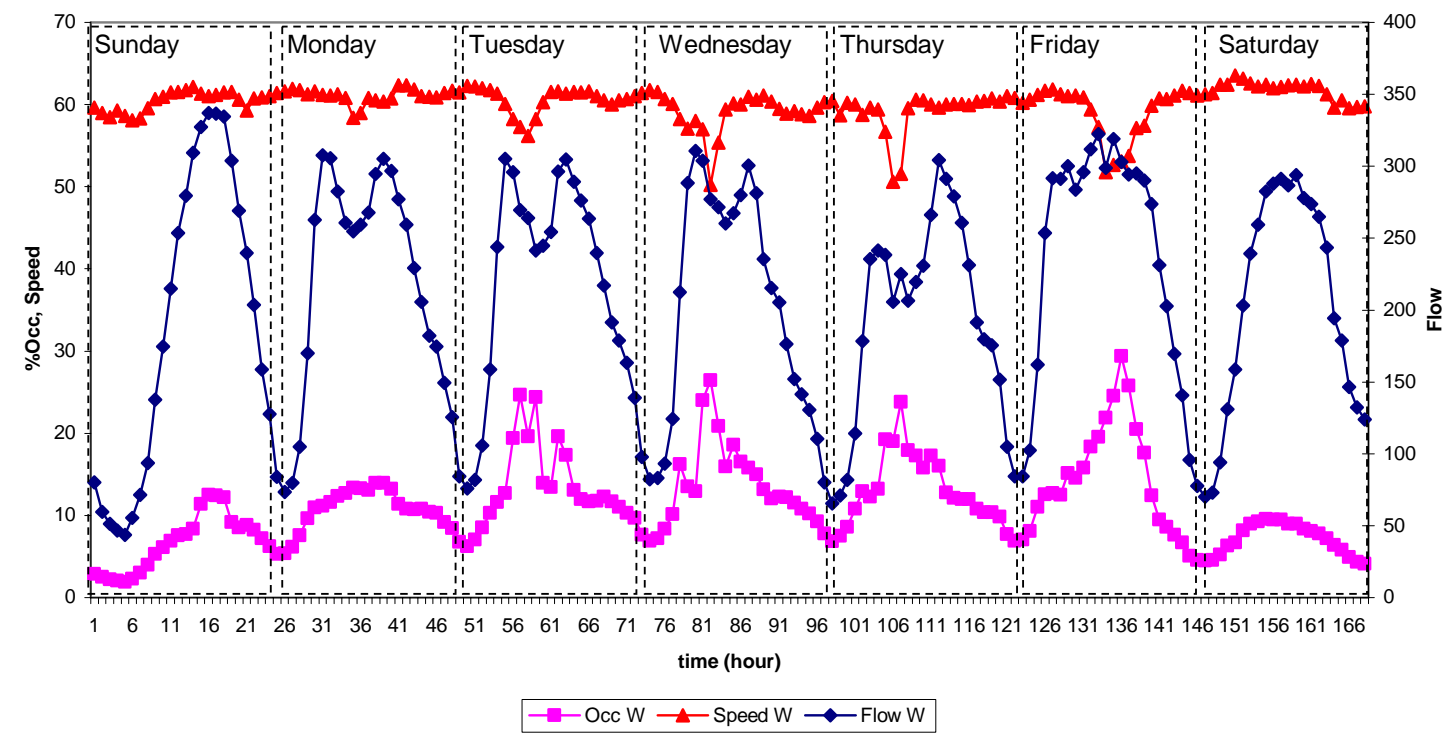

Figure 3.2: March to May 2002 combined one week sample of traffic parameters time series of west bound (W) at the Indianapolis Blvd exit.

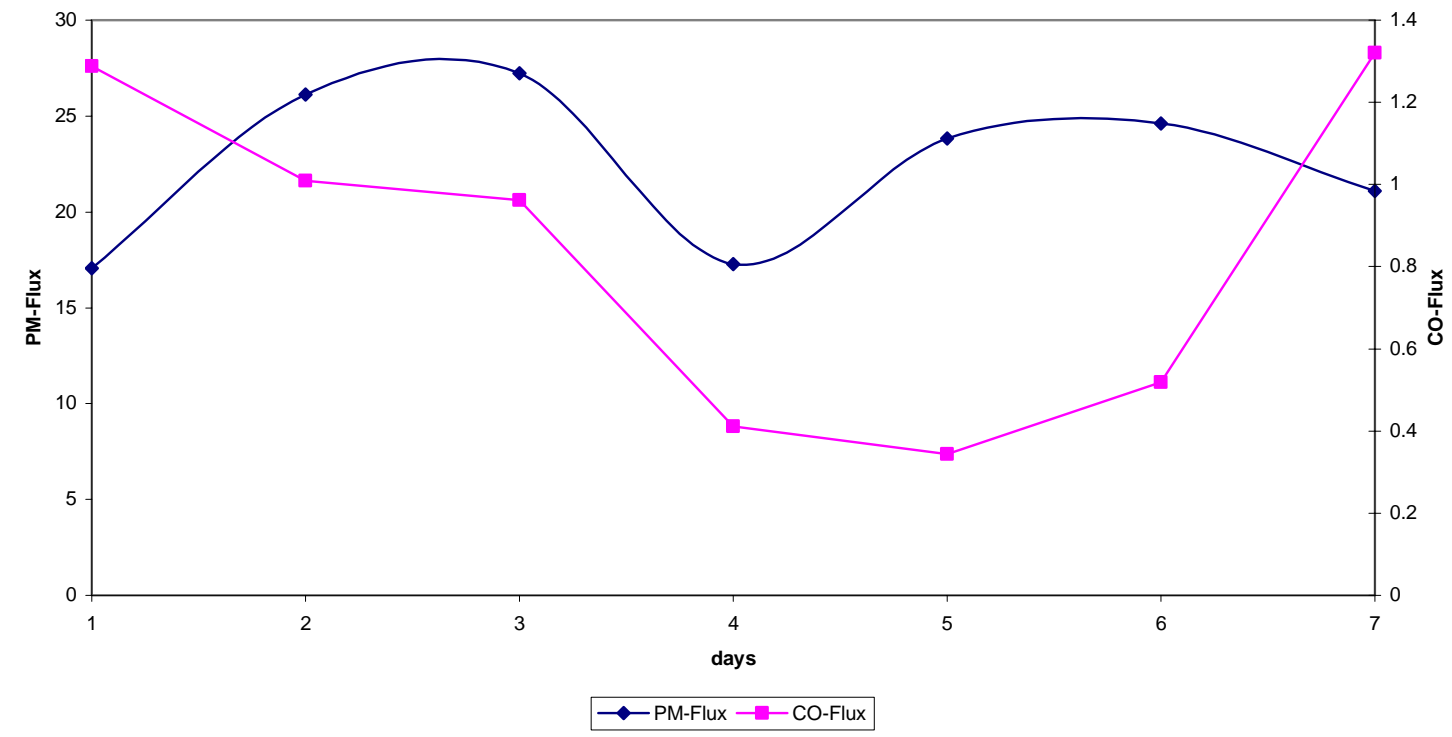

Figure 3.3: May 2002 combined daily averages data sample of PM2.5 and CO flux time series. 


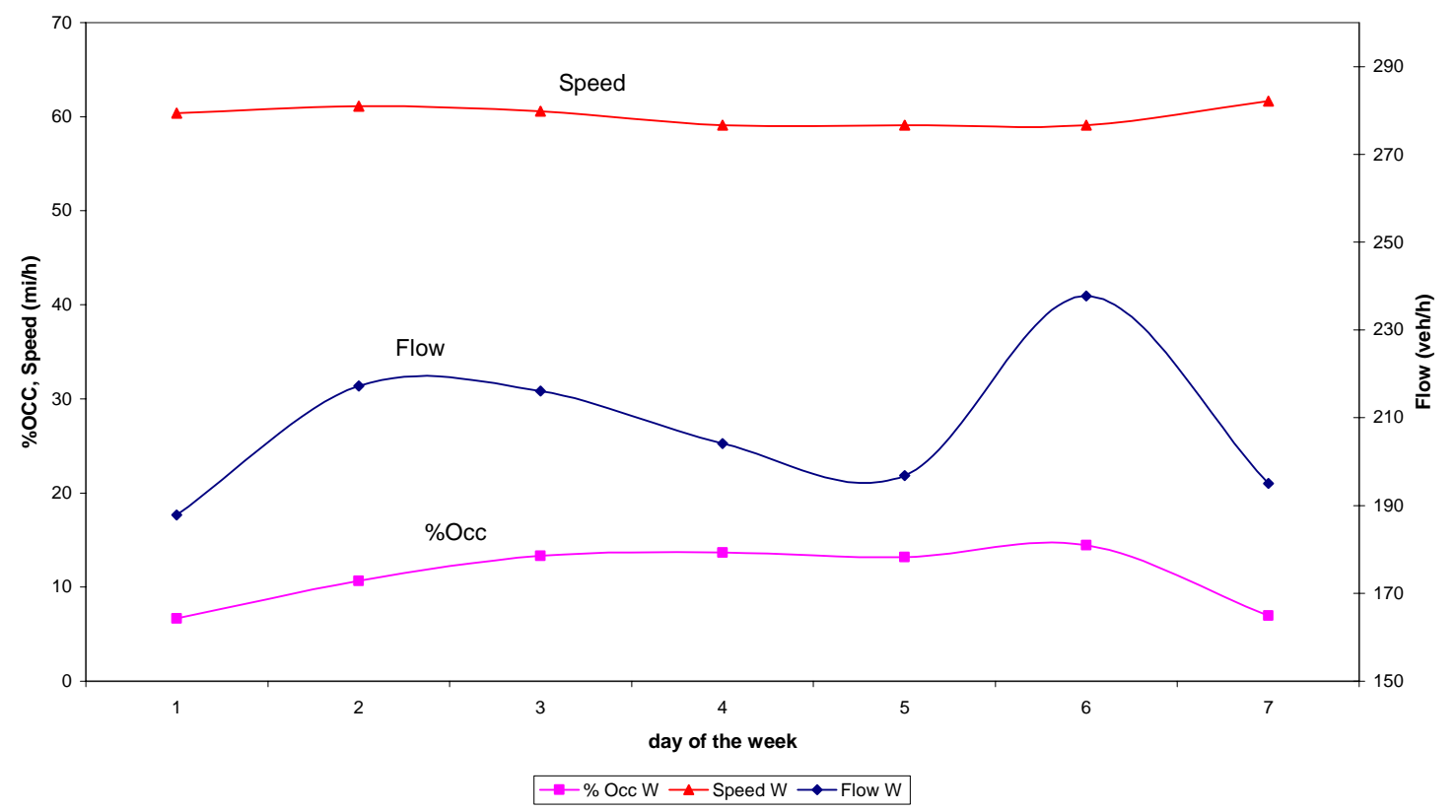

Figure 3.4: March to May 2002 combined daily average data sample of traffic parameters time series of west bound (W) at the Indianapolis Blvd exit.

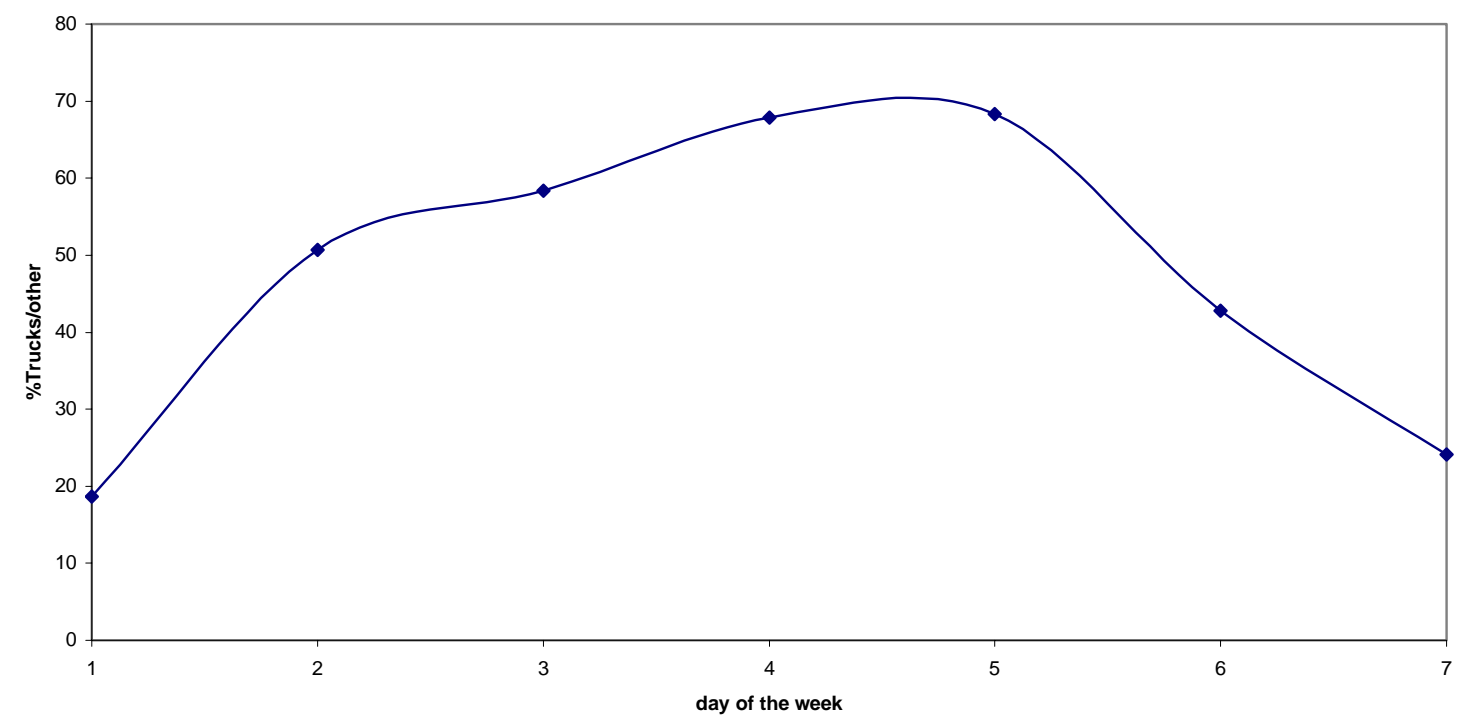

Figure 3.5: March to May 2002 combined daily average data sample of \% ratio of class 9-trucks to other vehicles time series of west bound at WIM station east of Site.

Elementary Spectral-Analysis in the frequency domain using Fast Fourier Transform (FFT) has also suggested a weekly and daily periodicity. Figure 3.6 shows an FFT analysis performed on the \%Occupancy data (as an example of the measured periodic data, other traffic and environmental parameters showed similar characteristics) with peaks corresponding to a 24- 
hour and 7-days periods. The yearly- or seasonal-trends can be calculated looking at full year's worth of monthly-averaged data.

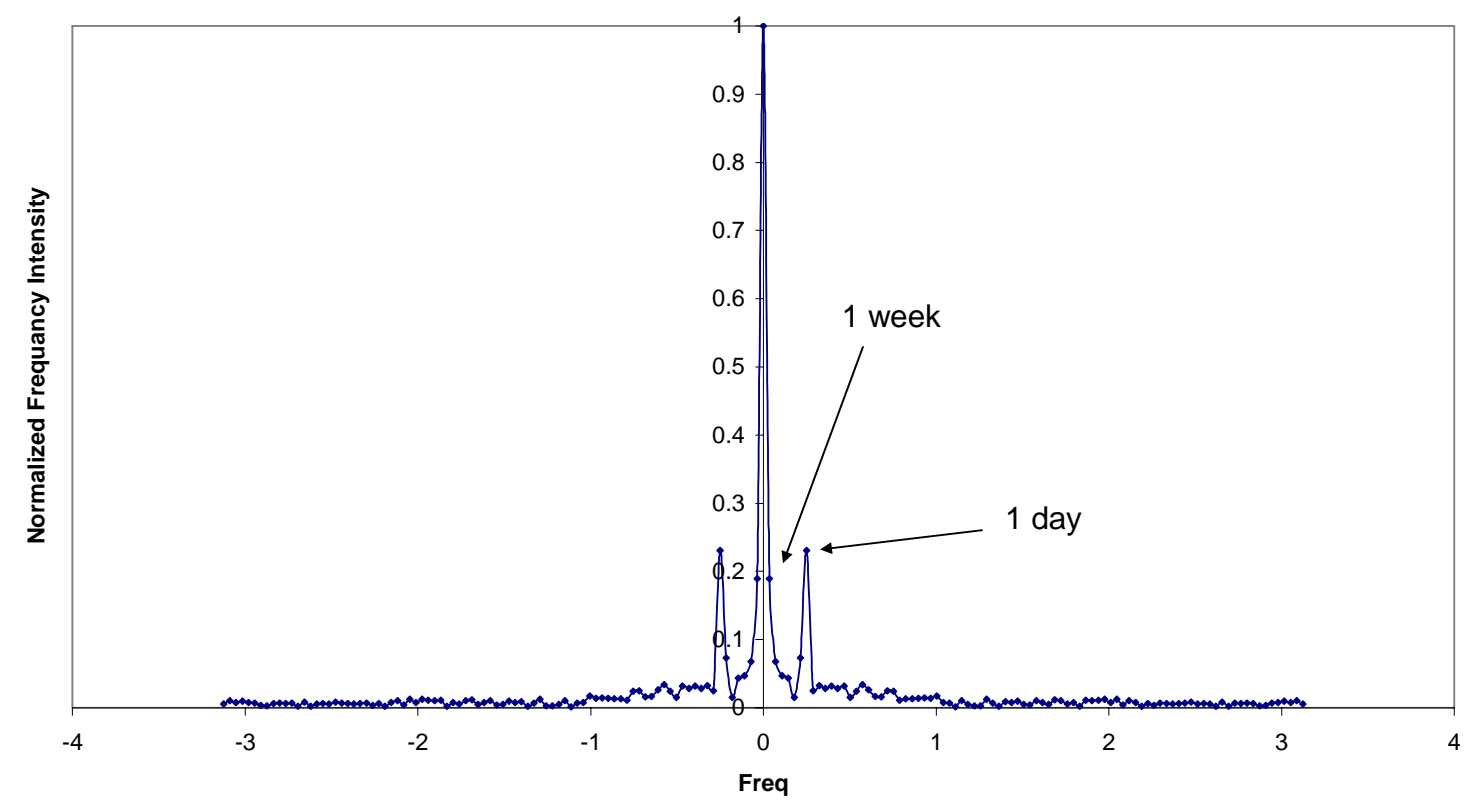

Figure 3.6: The Fast Fourier Transform (FFT) spectral analysis diagram of \% occupancy data showing peaks corresponding to the daily and weekly periods.

While a Time Series Analysis (TSA) is being performed on this database for the future (tools are illustrated in the Appendix), a cluster analysis has been performed with the traffic and environmental data. This report will focus solely on the cluster analysis to characterize (using a classification tree) the Borman Expressway local air quality as a function of traffic variables. 


\section{CLUSTER ANALYSIS STATISTICAL RESULTS AND METHODOLOGY}

A descriptive model has been developed to classify the Borman-traffic levels in terms of the ambient air quality levels (clusters). The cluster analysis study has a robust dataset because of the extended duration of sampling from January 2002 to June 2002, the use of ten-second average intervals for environmental conditions and the implementation of real-time WIM data and fiveminute loop data. In addition to the six-month sampling duration of this study, traffic conditions vary along the Borman Expressway with respect to the time of day. In this study, the dataset is divided into two different time periods. The first time period is between the daytime hours of 6:00 AM EST and 8:00 PM EST. This 14-hour daytime sampling period monitored the effects from morning, midday and afternoon rush hour periods. The second time period is in the nighttime between the hours of 8:00 PM EST and 6:00 AM EST. The evening period is used to show a baseline or best-case scenario with the lowest traffic volumes for the day.

There is approximately 30,000 five-minute intervals recorded, which allows for a dataset capable of accounting for high and low emitters (the top 5\% and bottom 5\% of vehicles); different ages and classifications of vehicular fleets; and different speeds, occupancies, truckloads, and dynamic environmental parameters. The developed model has an advantage over other simulation models since it is based on robust measured data from the Borman Expressway, hence; provide more realistic output and consequently more realistic analysis.

The developed model sampling duration allows for a wide range of environmental conditions, including different wind directions, ambient temperature, relative humidity, and rain. However, due to the close proximity of the mobile-air-quality monitoring station to the Borman Expressway, some meteorological parameters such as temperature and mixing height have minimal effect on the pollutant concentration data.

The combination of both traffic directions, in conjunction with appropriate wind directions, quantifies the ultimate effect from the Borman Expressway on local ambient air quality. For example, the eastbound and westbound lanes may contain high levels of congestion, which produce poor levels of ambient air quality, while another traffic condition might be free flowing with low traffic volumes in both directions, producing a cleaner or higher level of air quality. In either case, the impact on air quality is significantly different between best and worstcase scenarios (p-value < 0.0001). In addition to the cumulative emission effects from the east and west Borman Expressway mobile sources, the eastbound and westbound traffic is 
independent. Independence means that westbound traffic is not affected by traffic flow in the eastbound direction or simply that the westbound lanes might be congested while the eastbound lanes might be under free flow conditions. The independent nature from each direction means that one direction by itself cannot be used solely to predict conditions with the other direction.

\subsection{Model Selection Methodology}

The Borman Expressway is a dynamic freeway with conditions that can change from 110 to 10 vehicles per five-minute interval, occupancies that can increase from $10 \%$ to $50 \%$ and speeds that can decrease from $60 \mathrm{MPH}$ to less than $20 \mathrm{MPH}$ in a matter of a few minutes. With longer sampling time averages, there is a greater risk of missing statistically noteworthy incidents or greatly reducing the range of traffic parameters that occur during a period of congestion. For non-congested events, traffic conditions in some situations are highly variable as truck and car volumes change from morning rush hour to midday to afternoon rush hour and finally to low levels during the nighttime hours. The variability and the independence from both the eastbound and westbound lane directions along the Expressway create a highly dynamic dataset.

The classification development describing the effects from the Borman Expressway on ambient air quality involves several different statistical methodologies. These statistical methodologies were selected in order to determine the best method for this dataset. The first methodology selected was a traditional regression model developed to describe the entire dataset. The traditional regression model is defined by equation 3.1 .

$$
\mathrm{Y}_{\mathrm{i}}=\beta_{\mathrm{o}}+\beta_{1} \mathrm{X}_{1}+\ldots+\varepsilon_{\mathrm{i}}
$$

Where $\mathrm{Yi}$ is the response variable, $\beta_{0-\mathrm{i}}$ are the regression coefficients, $\mathrm{X}_{1-\mathrm{i}}$ are the predictor variables, and $\varepsilon_{\mathrm{i}}$ is the error. However, several problems arise when using a traditional regression model on this dataset.

The first problem is a result of multicolinearity with predictor variables. Multicolinearity is the result of two individual predictor variables that describe the same relationship. When both variables are selected for the regression model, one predictor variable becomes statistically significant, while the other predictor variable becomes statistically insignificant. One example of severe multicolinearity in the Borman dataset results from using both total eastbound truck counts, and eastbound class-nine truck counts. Therefore, total eastbound truck counts are deleted from the list of predictor variables. In other cases, with less multicolinearity, transformations on the predictor variables are done to minimize the effects from multicolinearity. 
The second problem associated with this dataset is the sample size and the variability. With a traditional regression model, the regression coefficients are very small, many times less than 0.001 with one example of this is the impact from eastbound class-nine volumes. In this example, eastbound class-nine volumes can be low during free flow conditions with low classnine volumes indicating a low truck density, the second condition with low class-nine volumes results from severe congestion, where traffic is not moving, which creates a high truck density. In these examples, the volumes are the same but the traffic patterns are distinctly different. In addition to the eastbound lanes, the additive effects from the westbound lanes are not provided. During this same period of time, the westbound lanes have a wide range of traffic conditions that are not described by the eastbound class-nine regression coefficient. For example, in addition to two different traffic patterns that are associated with the low eastbound class-nine volumes, there are many different westbound conditions that occur at the same time and all of these conditions affect the response variable. The large combinations of outcomes that are associated with each predicator coefficient create low regression coefficients. To curb this problem the interactions between different predictor variables are included in the traditional regression model. The interactions between the different predictor variables create the third problem associated with a traditional regression model. Interactions between variables are important because they help to better describe the relationship between predictor variables and the response variable. Discussed earlier, low number eastbound class-nine truck volumes, are a direct result of free flow conditions, or heavy congestion with the interaction with eastbound speeds the traffic description becomes clearer. High speeds and lower volumes suggest free flow conditions, while low speeds and low volumes suggest congestion. This is an example of a two-way interaction. In addition to two-way, higher-level interactions are also considered. For example, Equation 3.2 shows the problems that are associated with higher-level traditional regression interactions.

$$
Y_{i}=\beta_{0}+\beta_{1} X_{1}+\ldots+\beta_{123} X_{\text {east count, east speed, east occ. }}+\ldots+\varepsilon_{i}
$$

For example, in equation 3.2, there is a three-way interaction between eastbound class-nine count, eastbound speed, and eastbound occupancy. In this case the three-way regression coefficient is hard to interpret, in regards to the sign that is associated with the three-way interaction. As seen in the literature, physically, as the eastbound occupancy increases, the $\mathrm{PM}_{2.5}$ flux will increase, as the eastbound speed increases, the $\mathrm{PM}_{2.5}$ flux will decrease, and as the eastbound occupancy increases, the $\mathrm{PM}_{2.5}$ flux will increase. If this traffic condition occurs, two of the three predictor 
variables increase $\mathrm{PM}_{2.5}$ flux, while the third predictor variable decreases $\mathrm{PM}_{2.5}$ flux. This creates interaction regression coefficients that are hard to individually interpret. Because of the traffic variables and the independence associated with both directions, four and five way interactions between the different traffic patterns are included in the final regression model. The higher level interactions in conjunction with the original predictor variables create a traditional regression model, which includes over 100 predictor and interaction variables. In addition to the large size of the model, the interpretation of the interaction regression coefficients and in some cases very small values for regression coefficients, the final adjusted $\mathrm{R}^{2}$ remains very small, i.e., in most cases less than 0.5. Due to the inefficiency and the interpretability of the single regression model, it is not considered the ideal statistical methodology.

The second statistical methodology is comprised of eight different subgroups from the original dataset that are used to develop eight different multiple regression models. For example, one model for congestion periods in each direction; a second model for free flow eastbound and congestion westbound; and the remaining models accounting for the six other possible eastbound and westbound combinations. By dividing the original dataset into smaller subsets, the individual models are more efficient because regression coefficients describe a smaller section of data. In this case Equation 3.1 is able to change regression coefficients to better represent the eight smaller datasets, allowing for a better-fitted model. Nevertheless, the multiple regression models include the same problems with complicated interactions, large predictor variables and low adjusted $\mathrm{R}^{2}$ values. Although these models perform better than a single regression model, it is decided that there are better techniques for elucidating the Borman Expressway dataset.

With the variability in the dataset, two different statistical data mining techniques, called bootstrapping and bagging were selected. Bagging randomly draws datasets of the same size from the original dataset and creates a new training set. The new training set is refitted to develop a statistical model based on that particular training set and each training set models are compared with each other (Hastie et. al., 2001). In some cases bagging may occur 100 times with 100 different training sets and 100 different models. By creating many training sets, bagging in some cases, decreases the overall prediction error associated with a model. The other mining technique, bootstrapping, is similar by decreasing the prediction error by compiling many different datasets in order to smooth the different trends. This method like the bagging method is not used because the coefficients are difficult to interpret, creating a black box model.

The final selection was a classification tree model in conjunction with cluster analysis. A classification tree was selected because it is an efficient way to analyze this dataset. In this case, 
instead of a regression model with many predictor variables or a black box method, both of which are hard to interpret individual regression coefficients, a classification tree allows for the interpretation of complex interactions because of its hierarchical shape. Traditional classification regression trees including ID3, C4.5 and C5.0 use different algorithms to derive different rules for splitting different individual nodes (Hastie et al. 2001). Because of the variability associated with the Borman Expressway dataset, manual inspection is used to define the different node separations and clusters. To determine the appropriate separation of the nodes, a cluster analysis on the traffic parameters were done to determine both hierarchy and boundary conditions associated with each node separation. A cluster analysis method groups data of a similar statistical and physical nature together, creating different clusters. The cluster analysis approach capitalizes on both periods of rapid change, as well as stable conditions along the Borman Expressway. A study by Niemeier in February of 2002 suggested this cluster analysis method was an effective optimization of traffic data to create a hierarchy capable of comparing traffic data and in his case ozone data (Niemeier et. al, 2002). The remaining sections in this chapter will develop the different traffic boundary conditions that are used to create the 16 statistically different clusters used in the descriptive model.

\subsection{Selection of Traffic Patterns}

\subsubsection{Total Truck Volume vs. Class-Nine Truck Volume}

Figure 4.1, total truck count vs. class-nine truck count for both eastbound and westbound directions, is generated using over 5,960 five-minute averages for a time period between January 2002 and June 2002. These intervals include all levels of traffic conditions, ranging from free flow to heavy congestion. For all lanes eastbound and westbound, the $\mathrm{X}$-axis is the total classnine truck volume and the $\mathrm{Y}$-axis is the total truck volume. Due to the volume of points that are represented in figure 4.1, only the eastbound and westbound trend lines with $\mathrm{R}^{2}$ and equations are shown. The correlation between class-nine volumes and total truck volumes are very similar for eastbound and westbound directions with slopes of 1.2, as well as $\mathrm{R}^{2}$ values of 0.96 and 0.97 . During periods where there are no class-nine trucks, on average, the eastbound direction will have 7 non-class-nine trucks and the westbound direction will have 5 non-class-nine trucks per fiveminute sampling interval. 


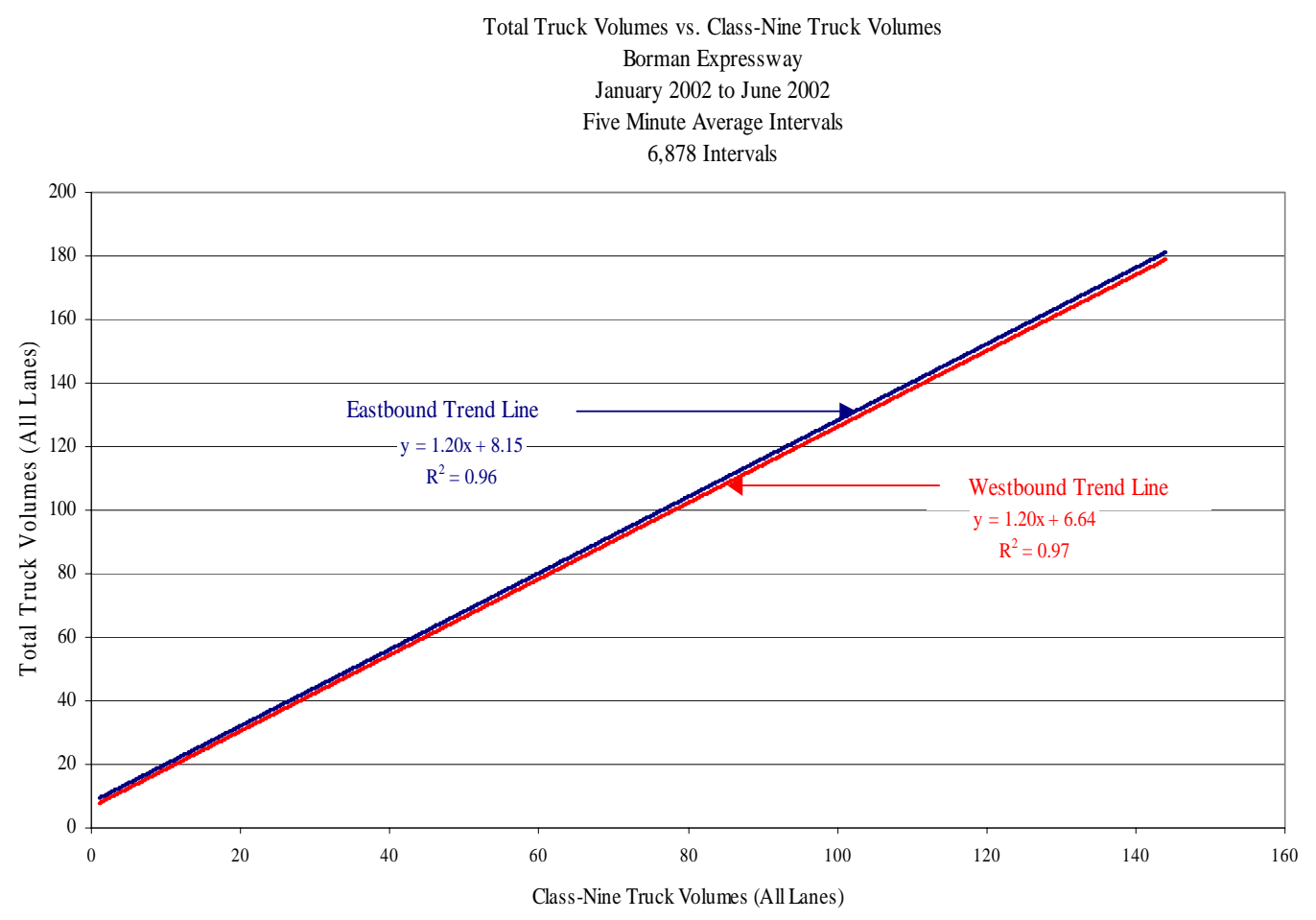

Figure 4.1: Total Truck Volumes vs. Class-Nine Truck Volumes.

Non-class-nine trucks range from school buses to multiple trailer trucks). Total volumes may be interchanged with class-nine volumes by using Equations 3.3 and 3.4.

$$
\begin{array}{ll}
\text { Total Eastbound Volume }=1.2 * \text { Class } 9+6.59 & 4.3 \\
\text { Total Westbound Volume }=1.2 * \text { Class } 9+5.37 & 4.4
\end{array}
$$

\subsubsection{Total Truck Speeds vs. Class-Nine Truck Speeds}

Class-nine volumes are the largest component of the truck fleet mix on the Borman; consequently, the class-nine average speeds are highly correlated with the average total fleet speeds. Figure 4.2 shows the correlation between the average total truck speeds vs. the average class-nine speeds. The $\mathrm{X}$-axis is the class-nine average speed and the $\mathrm{Y}$-axis is the total truck average speed (units are MPH). There are two different trend lines, one eastbound and one westbound and for both cases the slope is 1.01 with a $\mathrm{R}^{2}$ equal to 0.99 . 


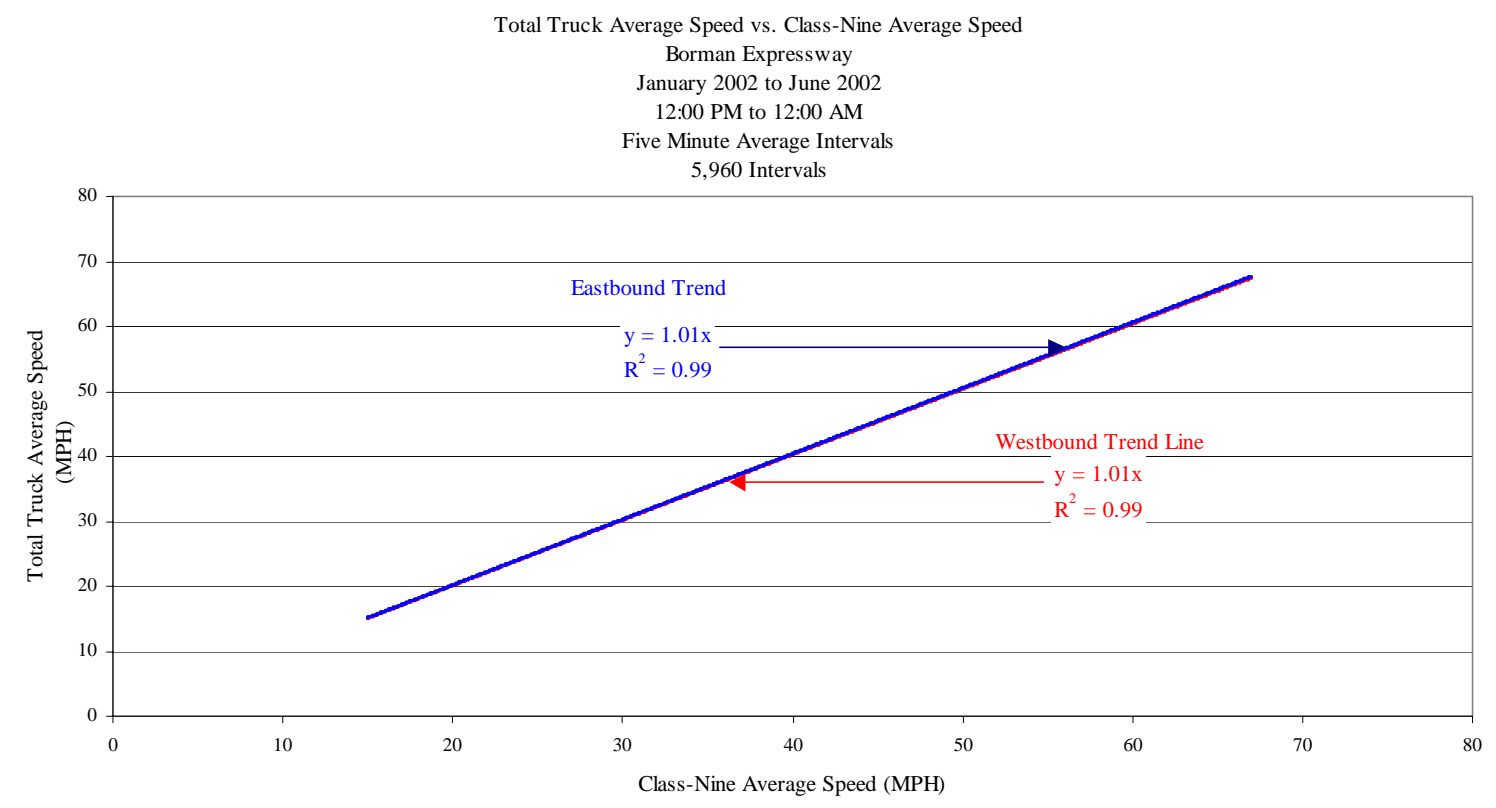

Figure 4.2: Total Truck Average Speed vs. Class-Nine Average Speed.

The high correlation between the total truck average speeds and the class-nine average speeds suggest that class-nine trucks have a great influence on the overall average fleet speed on the Borman. It is very unlikely, for class-seven trucks to average speeds in the $70 \mathrm{MPH}$ range when class-nine trucks are traveling in the $50 \mathrm{MPH}$ range.

The relationship between the class-nine traffic conditions and total truck conditions as seen in Figures 4.1 and 4.2 are nearly equivalent; thus, for the purpose of this study, class-nine conditions are used to represent the total traffic conditions associated with truck patterns.

\subsection{Operational Conditions Along the Borman Expressway}

\subsubsection{Traffic Occupancy vs. Class-Nine Truck Volumes}

Comprised of 17,739 five-minute intervals, Figure 4.3 shows the correlation between eastbound occupancy and class-nine truck volumes for daytime operational hours. The X-axis is the eastbound class-nine truck volumes and the Y-axis is the eastbound occupancy. 


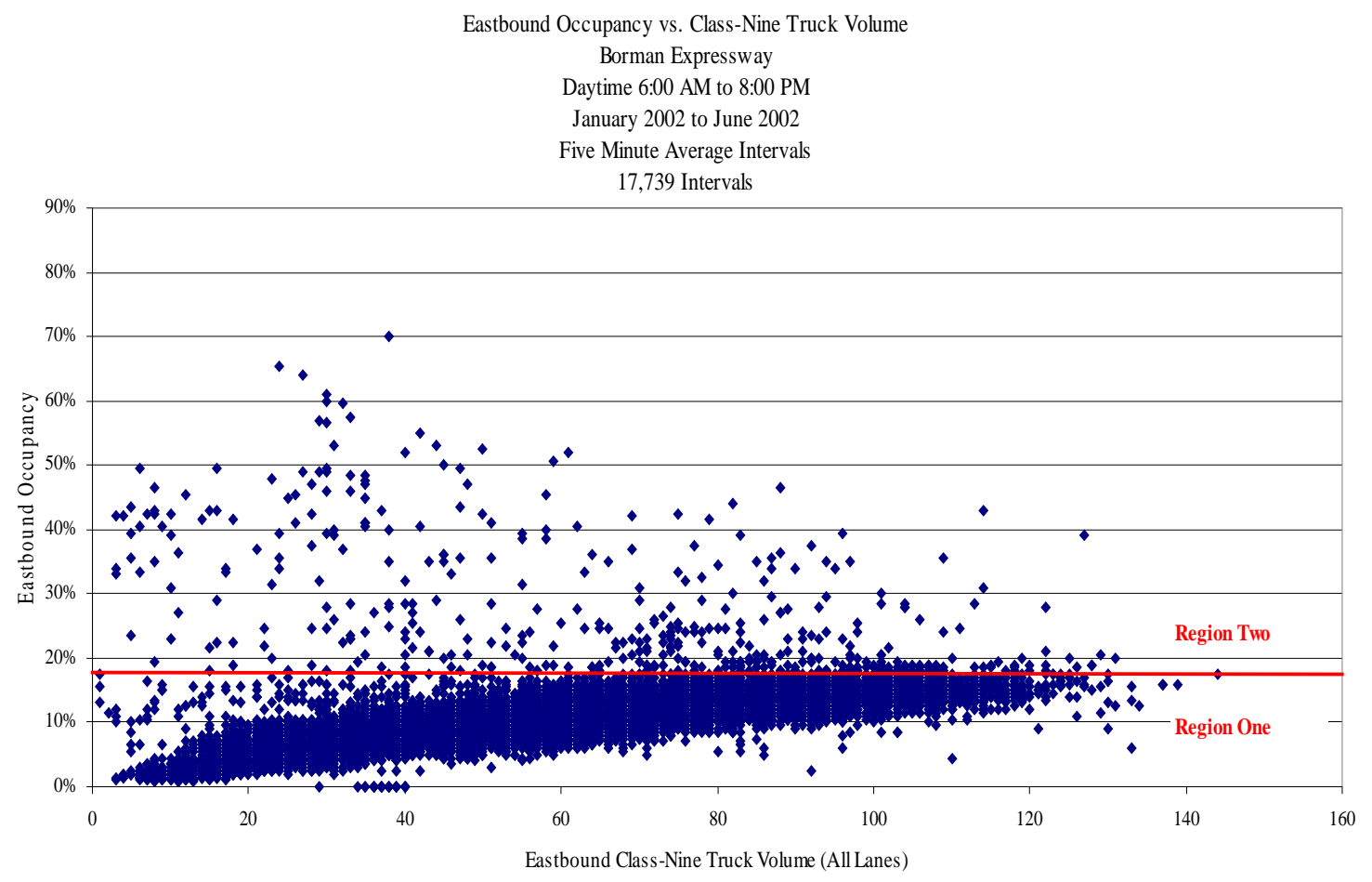

Figure 4.3: Eastbound Occupancy vs. Class-Nine Truck Volumes.

Figure 4.3 contains the entire dataset where traffic loop data and WIM information are collected. There is no filtration of traffic data based on environmental conditions; in other words, the traffic dataset is not affected by wind direction.

There are two different regions to this graph. Region One has occupancies less than or equal to $18 \%$, while Region Two has occupancies greater than $18 \%$. Region One, which contains approximately $97.6 \%$, or 17,326 intervals, shows a moderate trend $\left(\mathrm{R}^{2}=0.67\right)$ between the increase in class-nine volumes and the increase in overall occupancies. During this normal operation, the increase in occupancy is a direct result of the amount of "on time" associated with each vehicle. The change in traffic volumes from less than 20 to greater than 100 vehicles is explained by the changing traffic activity that naturally occurs throughout the day. Different traffic patterns include morning, midday, and afternoon rush hours for example. These higher volumes associated with different rush hours create longer "on time" periods, increasing the overall occupancy along the Borman Expressway.

When the eastbound occupancy is higher than $18 \%$, the moderate linear trend between volumes and occupancies that is illustrated in Region One is not seen in Region Two and the $\mathrm{R}^{2}$ 
falls from 0.67 to 0.35 . The individual points associated with Region Two are separated and low class-nine truck volumes have occupancies exceeding 60\%. The increase in occupancies, with decreasing class-nine truck volumes, is explained by decreasing speeds. With lower speeds, the amount of "on time" for the loops is increased, suggesting more inefficient travel. In this case, when eastbound occupancies increase over 18\%, class-nine truck speeds on average decrease from $61 \mathrm{MPH}$ to $48 \mathrm{MPH}$ and the standard deviation of speeds increases from 4.4 MPH to 5.4 MPH. The combination of decreased truck volumes, speeds, and the increased standard deviation of speeds indicate the impact of traffic congestion. This inefficient travel causes higher engine loading stemming from periods of acceleration and deceleration and suggests higher emissions of particulate matter from trucks traveling in the eastbound direction (Metz et al. 1997, Yanowitz et al. 2002).

The westbound occupancy vs. class-nine truck volumes, illustrated in Figure 4.4, are generated in a similar manner as seen in Figure 4.3.

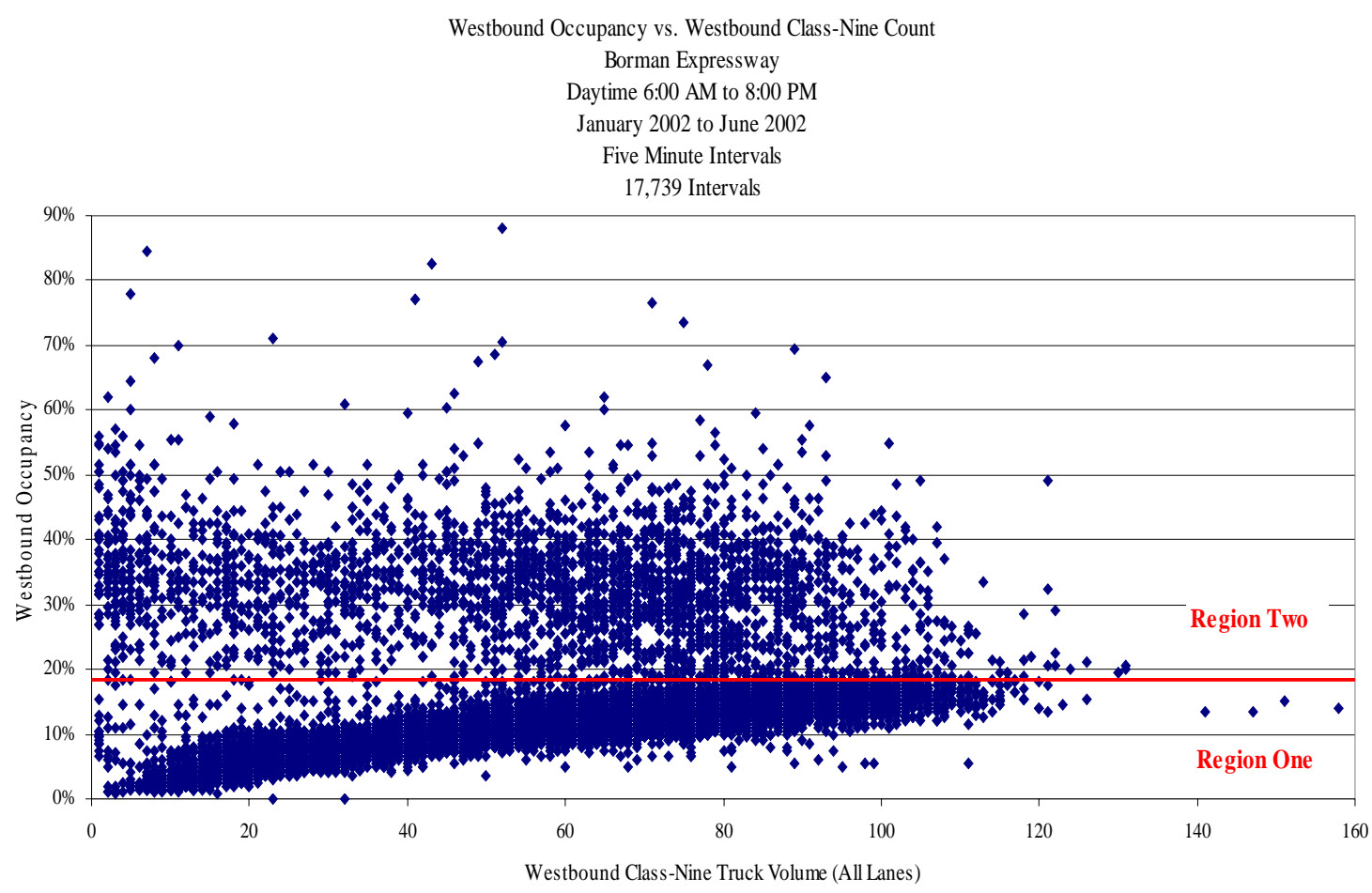

Figure 4.4: Westbound Occupancy vs. Westbound Class-Nine Volume 
As in Figure 4.3, Figure 4.4 has two distinct regions. Region One, includes occupancies less than or equal to $18 \%$ and Region Two has occupancies greater than $18 \%$. The same trend, consistent with the eastbound direction appears for occupancies in Region One. In this case 84\%, or 14,933 individual intervals, are contained in region one with a $\mathrm{R}^{2}=0.72$. The same explanation used for the Region One eastbound trend may be applied here.

In Region Two, above 18\% westbound occupancy, there is a large amount of scattering and the $\mathrm{R}^{2}$ drops from 0.72 to 0.06 . As the occupancy increases above $18 \%$, the average classnine speed decreases from $60 \mathrm{MPH}$ to $49 \mathrm{MPH}$ and the standard deviation of speed increases from 4.3 MPH to 5.4 MPH. The decrease in average speed, in conjunction with an increase in the standard deviation of speed, indicates the effects from congestion along the Borman Expressway, placing higher engine loading on westbound trucks creating higher emissions of particulate matter (Metz et al. 1997, Yanowitz et al. 2002).

\subsubsection{Eastbound and Westbound Traffic Occupancy vs. Traffic Volume Comparisons}

When Figures 4.3 and 4.4 are compared with each other, there is a clear difference in the amount of scattering above $18 \%$ total occupancy. Where occupancies are greater than $18 \%$, the eastbound directions include 413 five-minute intervals, while the westbound direction includes 2,806 five-minute intervals. The westbound direction has 2,393 more five-minute intervals that are greater than $18 \%$. This difference is explained by the bottlenecks created by the area surrounding the city of Chicago. Since the Borman Expressway is a major expressway traveling east and west and the city of Chicago is to the west of the sampling location, traffic in the westbound direction will naturally continue to increase in volume. This increase in traffic volume creates higher traffic demands and greater probabilities for congestion and inefficient travel in the westbound direction.

The opposite traffic pattern occurs in the eastbound direction. Here, traffic is moving away from Chicago and the further east on the Expressway, the more significant the decrease in total traffic volume. The decrease in volume lowers the traffic demand along the Expressway, thus decreasing the frequency for congestion.

\subsubsection{Traffic Occupancy vs. Class-Nine Average Speeds}

In addition to traffic occupancies vs. class-nine volumes, traffic occupancies can be directly compared to class-nine average speeds. Figure 4.5 compares the eastbound occupancy vs. the eastbound class-nine average speed during daytime hours of operation. Figure 4.5 includes two different boxes or groups containing $97.5 \%$ of the entire dataset. Group one consists of 16,609 five-minute intervals where the Borman Expressway is operating under normal 
conditions. Normal conditions include average class-nine truck speeds greater or equal to 50 $\mathrm{MPH}$ and total occupancies less than or equal to $18 \%$. An 18\% occupancy limit is selected based on the results from Figure 4.3 and the scattering that occurred when the average eastbound occupancy increases above 18\%. The $50 \mathrm{MPH}$ speed limit is selected because it is within $5 \mathrm{MPH}$ of the posted 55 MPH speed limit for the Borman Expressway. Speeds greater than $55 \mathrm{MPH}$, even though they exceeded the posted speed limit, are acceptable for normal operating conditions.

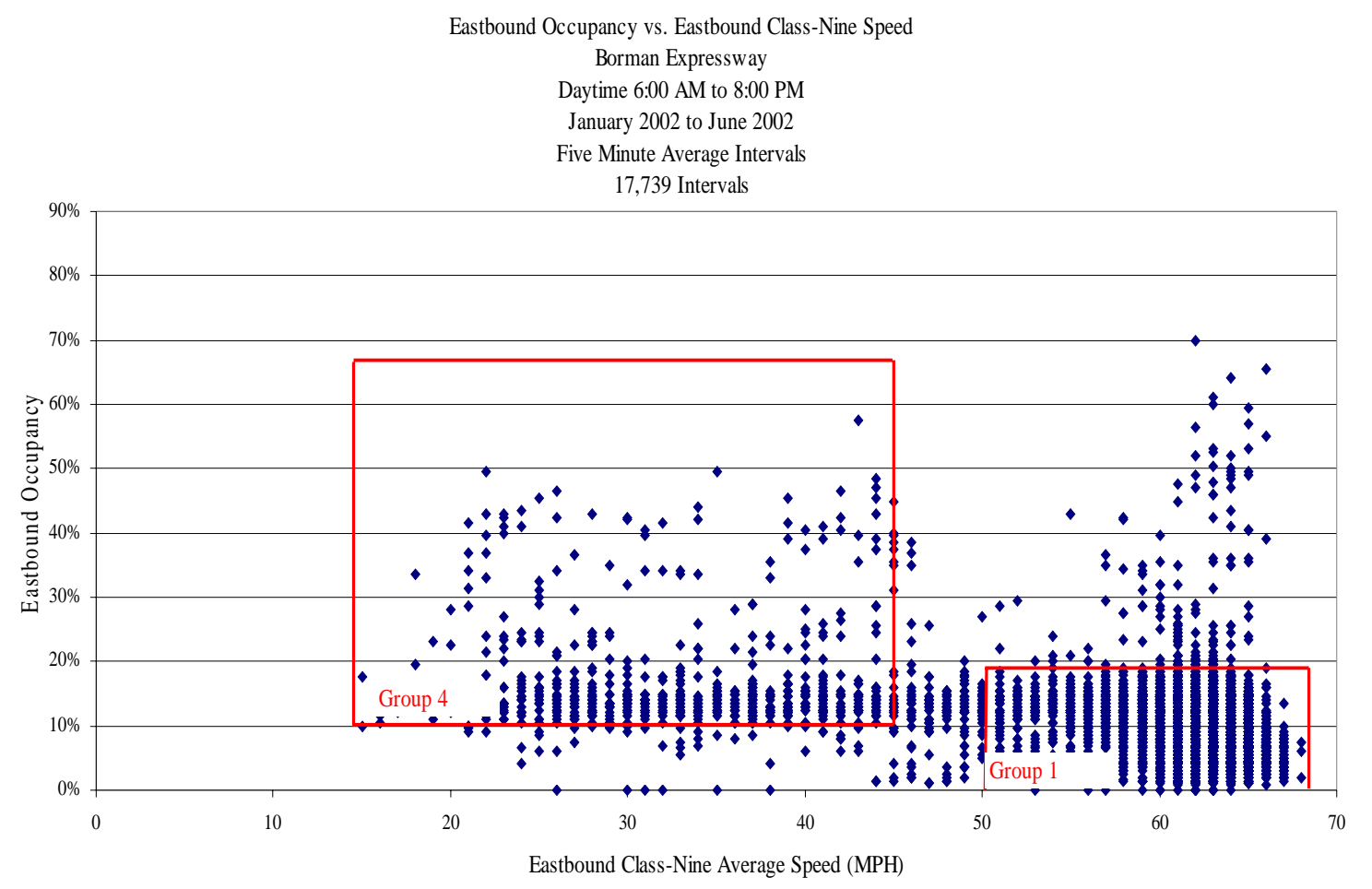

Figure 4.5: Eastbound Occupancy vs. Eastbound Class-Nine Speed.

The second box, group four, consists of 696 five-minute average intervals which include speeds less than or equal to $45 \mathrm{MPH}$ and eastbound occupancies greater or equal to $10 \%$. Occupancies lower than $18 \%$ are included to increase the sample size from 157 to 696 intervals. In addition to enlarging the sample size, lowering the occupancy boundary condition from $18 \%$ to $10 \%$ provide for a more conservative final ambient predictor of $\mathrm{PM}_{2.5}$ under heavy traffic conditions.

Group four upper average speed boundary condition is a minimum of $10 \mathrm{MPH}$ lower than the posted speeds for the Expressway; therefore, the Expressway is not operating under design 
conditions. These lower speeds represent high congestion, creating increases and decreases in acceleration that cause a difference in engine loading between vehicles traveling at a constant 35 MPH on a secondary road (with a $35 \mathrm{MPH}$ posted speed limit) and vehicles traveling $35 \mathrm{MPH}$ on an Expressway. Speeds less than $50 \mathrm{MPH}$ and greater than $45 \mathrm{MPH}$ are not included because a clear separation between normal and congestion speeds must be established. The daytime westbound occupancy vs. westbound class-nine average truck speed, shown in Figure 4.6, includes the same $\mathrm{X}$-axis and $\mathrm{Y}$-axis parameters and sampling duration as shown in Figure 4.5.

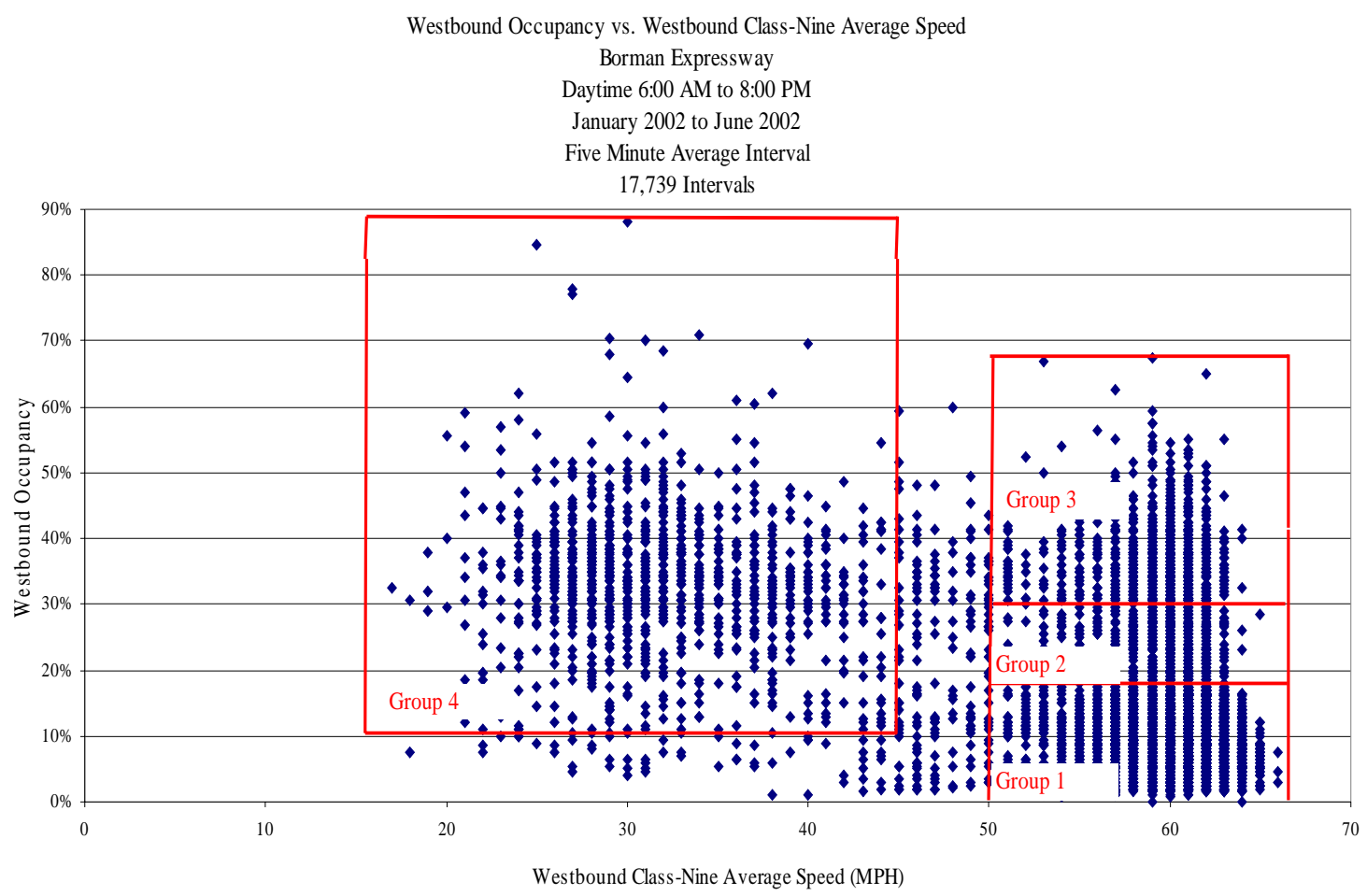

Figure 4.6: Westbound Occupancy vs. Westbound Class-Nine Speed.

Because the westbound traffic flow is different from the eastbound traffic flow two more groups are created. Groups one, two, three, and four contain $98.9 \%$ of the total five-minute scatter points in the westbound direction. Group one in Figure 4.6 has the same operating conditions as group one in Figure 4.5, with speeds greater or equal to $50 \mathrm{MPH}$ and occupancies less than or equal to $18 \%$. On average, there are 57 class-nine vehicles per five-minute interval during a group one set of boundary conditions.

Group two is the same 50 MPH or greater average speeds with occupancies greater than $18 \%$ and less than or equal to $30 \%$. Occupancy of $30 \%$ is selected as the boundary condition in 
order to provide group two and group three with similar sample sizes (group two: 849, and group three: 925). Group two increases the average class-nine volume by 12 trucks to 69 class-nine trucks per five-minutes (p-value of 0.0001 for group one and group two). The increase in average class-nine volumes may be attributed to the time of day. Group two contains on average more time during higher peaks in daytime operation, including some periods during rush hours, where car and truck volumes are higher. The increase in car volumes is based on the hourly average volumes taken from the WIM.

The third boundary condition occurs when speeds are greater than $50 \mathrm{MPH}$ and the westbound occupancy is greater than $30 \%$. The average class-nine truck volume per five-minute average changes from 69 to 66 (p-value of 0.1). Based on the p-value, the difference between group two and group three is insignificant. So, the increase in occupancy on average is due to the increase in the amount of car volumes during these periods of time.

Although groups one, two and three have relatively similar average speeds, 61, 60, and $59 \mathrm{MPH}$ respectively, both car and truck volumes increase between the three different groups, creating more demand on the Expressway. The increased demand decreases the overall stability of the Expressway, creating possible congestion periods.

The fourth and final group is the same as group four in Figure 4.5, with speeds less than or equal to $45 \mathrm{MPH}$ and the westbound occupancy greater or equal to $10 \%$. A boundary of $10 \%$, as opposed to $18 \%$, is selected to allow for continuity for direct comparisons between the eastbound and westbound lanes. Unlike the eastbound direction, the decrease in occupancy from $18 \%$ to $10 \%$ is not used to increase the overall sampling size $(1,000$ to 1,098$)$.

\subsubsection{Summary of Eastbound and Westbound Groups}

In conclusion, there are four groups of boundary conditions in the westbound direction and two groups of boundary conditions in the eastbound directions. Groups one and four have the same traffic parameters for each direction, and groups two and three are used in the westbound direction. In total, the eastbound groups, one and four use $97.5 \%$ of the dataset and the westbound groups use $98.9 \%$ of the dataset.

\subsection{Traffic Incidents}

\subsubsection{Eastbound Incident}

Figure 4.7 occurred on April 30, 2002 between 9:00 AM and 3:00 PM EST. The X-axis is the time of day; the left Y-axis is class-nine average speeds and class-nine volumes for all 
eastbound lanes. The secondary Y-axis, located on the right side of the figure, is the Eastbound Occupancy.

Figure 4.7 includes three different lines: the dark line represents class-nine volumes, the lighter line represents eastbound occupancies, and the light-gray line represents class-nine average speeds. The first traffic pattern occurs between the hours of 9:00 AM EST and 10:30 AM EST. During this period of time the traffic flow on the Borman Expressway remains below normal (or a group one condition), with occupancies less than $18 \%$ and speeds greater than or equal to $50 \mathrm{MPH}$.

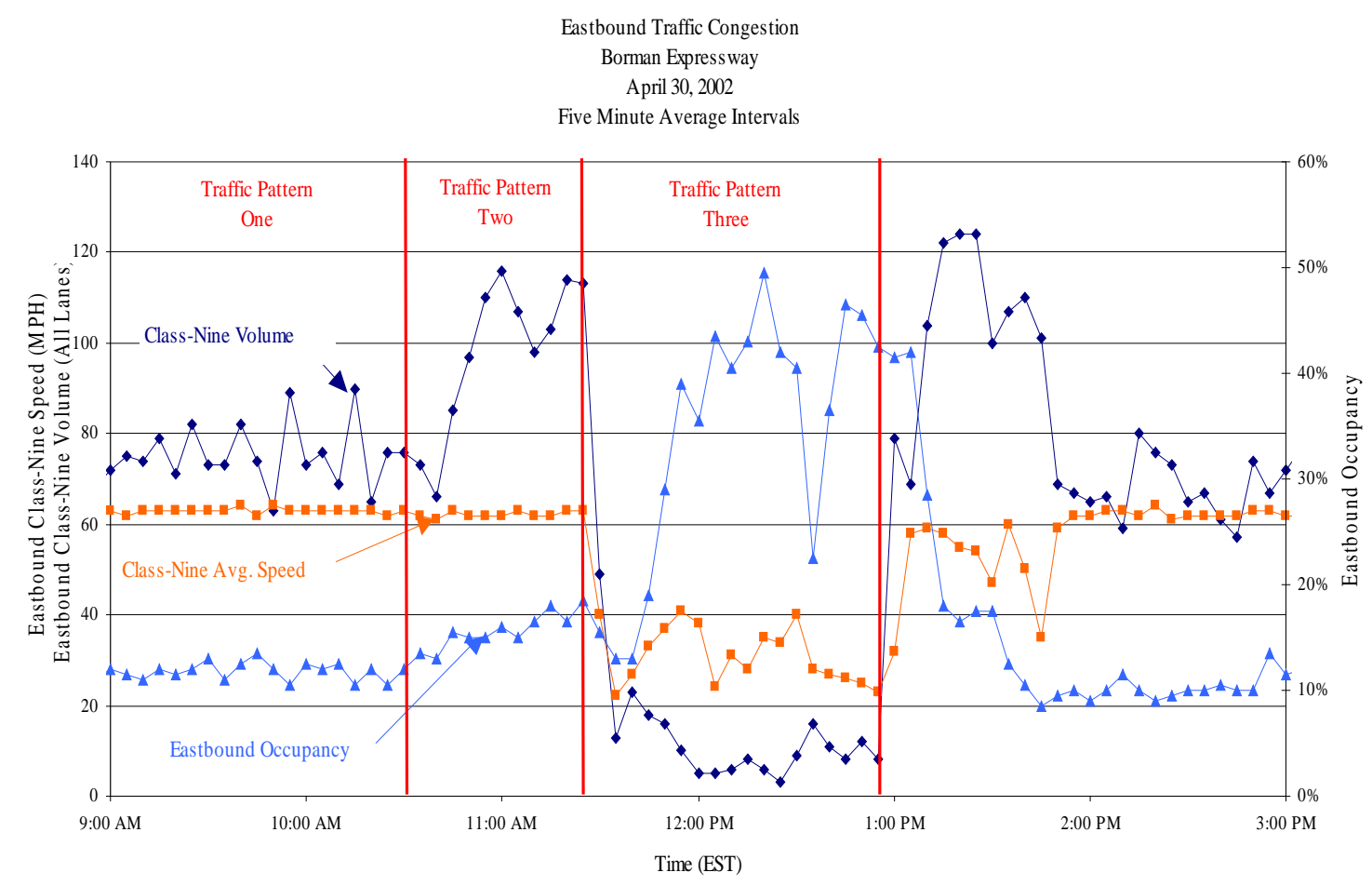

Figure 4.7: Eastbound Congestion Period, April 30, 2002.

Traffic pattern two occurs between 10:30 and 11:30 AM. During this time period the class-nine volume increase from approximately 75 to 110 vehicles per five minutes. The occupancy continues to rise from $10 \%$ to approximately $18 \%$. At this same time, the average speed remains constant, which suggests that the increase in occupancy is directly related to the increase in truck and car volumes. The increase in traffic demand creates the possibility for future congestion. 
Traffic pattern three occurs at 11:30 AM EST; the class-nine volume decreases from 110 class-nine trucks to less than 20 class-nine trucks per five-minutes. The class-nine truck average speeds also decrease from $60 \mathrm{MPH}$ to less than $40 \mathrm{MPH}$, with speeds declining as low as 22 MPH. During period three, from 11:30 AM EST to 1:00 PM EST, there is a sharp increase in occupancy from $18 \%$ to $40+\%$ with speeds less than $45 \mathrm{MPH}$ and the eastbound lanes are considered to be in-group four.

Around 1:00 PM EST the congested event begins to dissipate, restoring the capacity and allowing a large increase in truck volume and speeds. During this period the occupancy continues to decrease to normal levels and the Expressway goes back to a group one condition after 2:00 PM EST. This event is one traffic incident example in the eastbound direction; the scatter plots shown in Figures 4.3 and 4.5 represent all the traffic congestion periods from January 2002 to June 2002.

\subsubsection{Westbound Traffic Incident}

The westbound direction, Figure 4.8, also synthesizes the relationship between speeds, occupancies, and volumes. One example of this relationship is demonstrated on May 28, 2002 between 9:00 AM EST and 9:00 PM EST. The X-axis and Y-axis, as well as the three trend lines remain the same as in Figure 4.7.

Starting at 9:00 AM EST until 10:45 AM EST, conditions remain normal, a group one condition, with speeds greater than $50 \mathrm{MPH}$ and occupancies less than 18\%. There is one small interval of congestion around 10:15 AM EST, with a sudden drop in truck volumes and a slight rise in total occupancy. This period foreshadows the future congested period and suggests the possible instabilities in the westbound direction. Around 11:00 AM EST the occupancies increased to greater than 30\% with speeds remaining higher than $50 \mathrm{MPH}$. By 12:00 PM EST the congestion has reached a group four level with low speeds, low volumes, and high occupancies.

For the next six hours, the westbound direction remains under congestion with inefficient traffic flow, creating higher engine loadings and greater emission of $\mathrm{PM}_{2.5}$ (Metz et al. 1997, Yanowitz et al. 2002). Around 6:15 PM EST, the congestion on the roadway dissipates and normal traffic operations resume. All four westbound groups during the course of the day are represented in this graph. 


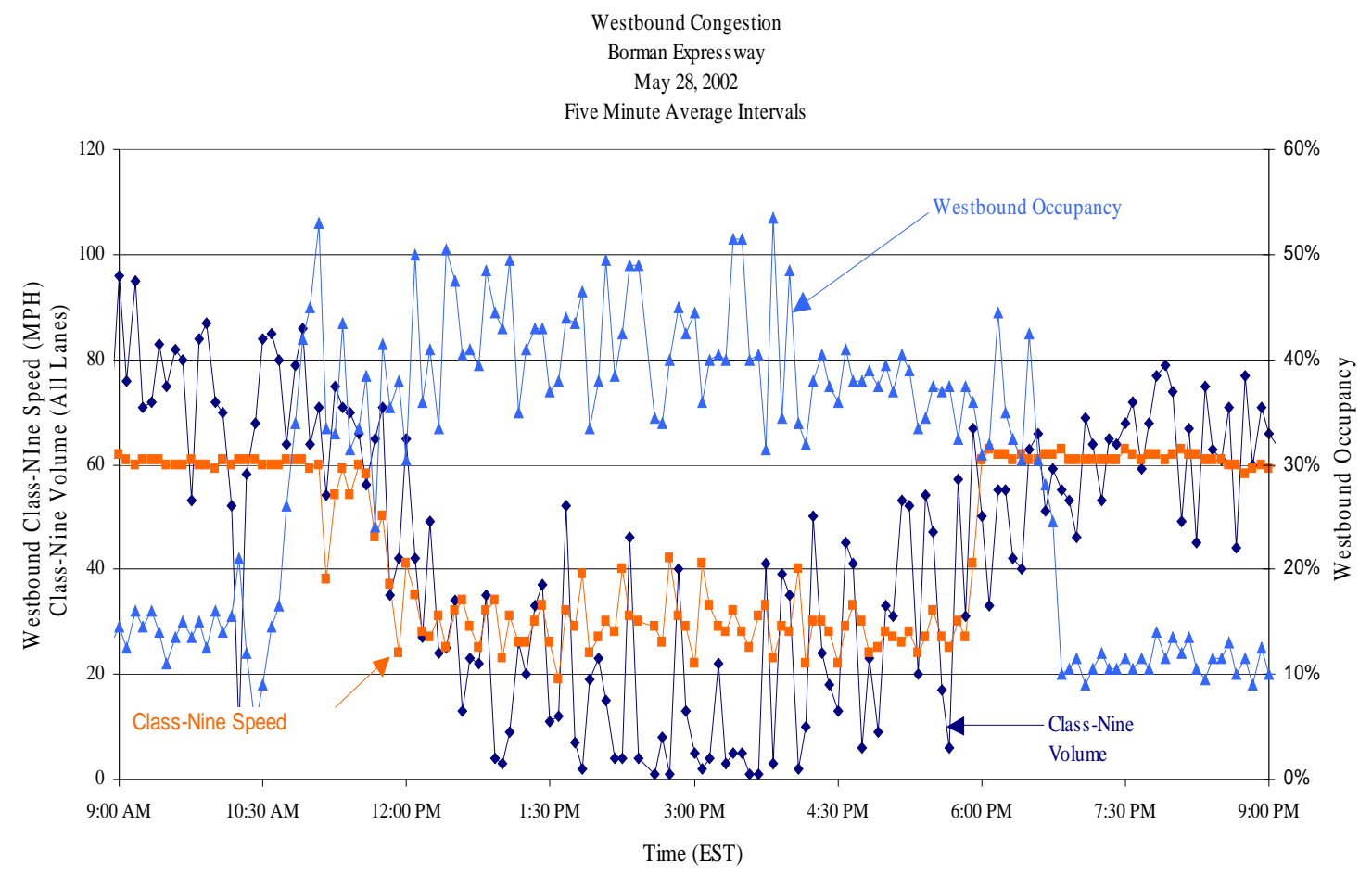

Figure 3.8: Westbound Congestion Period, May 28, 2002.

\subsection{Nighttime Operational Conditions on the Borman Expressway}

The nighttime traffic along the Borman Expressway is different from the daytime hours of operation. During the nighttime activity, there is on average lower volumes for both trucks and passenger cars. In this study, evening hours are between 8:00 PM EST and 6:00 AM EST. Similar to the daytime hours of operation, meteorological events are random. Section 3.6 will use the same grouping techniques that are used in Sections 3.3 and 3.4. In addition, to these grouping techniques class-nine trucks are used to represent the entire fleet mix.

\subsubsection{Nighttime Eastbound Occupancy vs. Class-Nine Truck Volume}

Figure 4.9 is a scatter plot of eastbound occupancy vs. class-nine truck volumes during the nighttime hours. The $\mathrm{X}$-axis is class-nine truck volumes on all lanes, and the $\mathrm{Y}$-axis is eastbound occupancy, and the time of day is from 8:00 PM EST to 6:00AM EST. The X-axis and the $\mathrm{Y}$-axis are the same as Figure 4.3. Similar to the daytime, there is a reasonably good correlation $\left(\mathrm{R}^{2}=0.65\right)$ between the increase in volume and occupancy with the dataset less then or equal to $18 \%$ occupancy. Using the whole nighttime dataset, the $\mathrm{R}^{2}$ is 0.57 , an increase from 0.35 
during the daytime hours, which is explained by the decrease in scatter points greater than $18 \%$ occupancy during the nighttime, suggesting less periods of nighttime congestion. The average volume of class-nine trucks decreases by 14 vehicles per five-minute interval between daytime and nighttime hours, as well as a decrease in hourly car volumes from a daytime volume of 2,514 to a nighttime volume of 1,009 .

The decrease in overall volume for both class-nine trucks and cars lowers the traffic demand on the Expressway. With a lower nighttime traffic demand, even large reductions in the capacity, such as a lane closure or a small accident, do not create a situation where the traffic demand is higher than capacity along the Expressway. Thus, the possibility of queues forming during the evening hours is minimal, as shown by the decrease from 413 intervals greater than $18 \%$ to less than 16 nighttime intervals greater than $18 \%$.

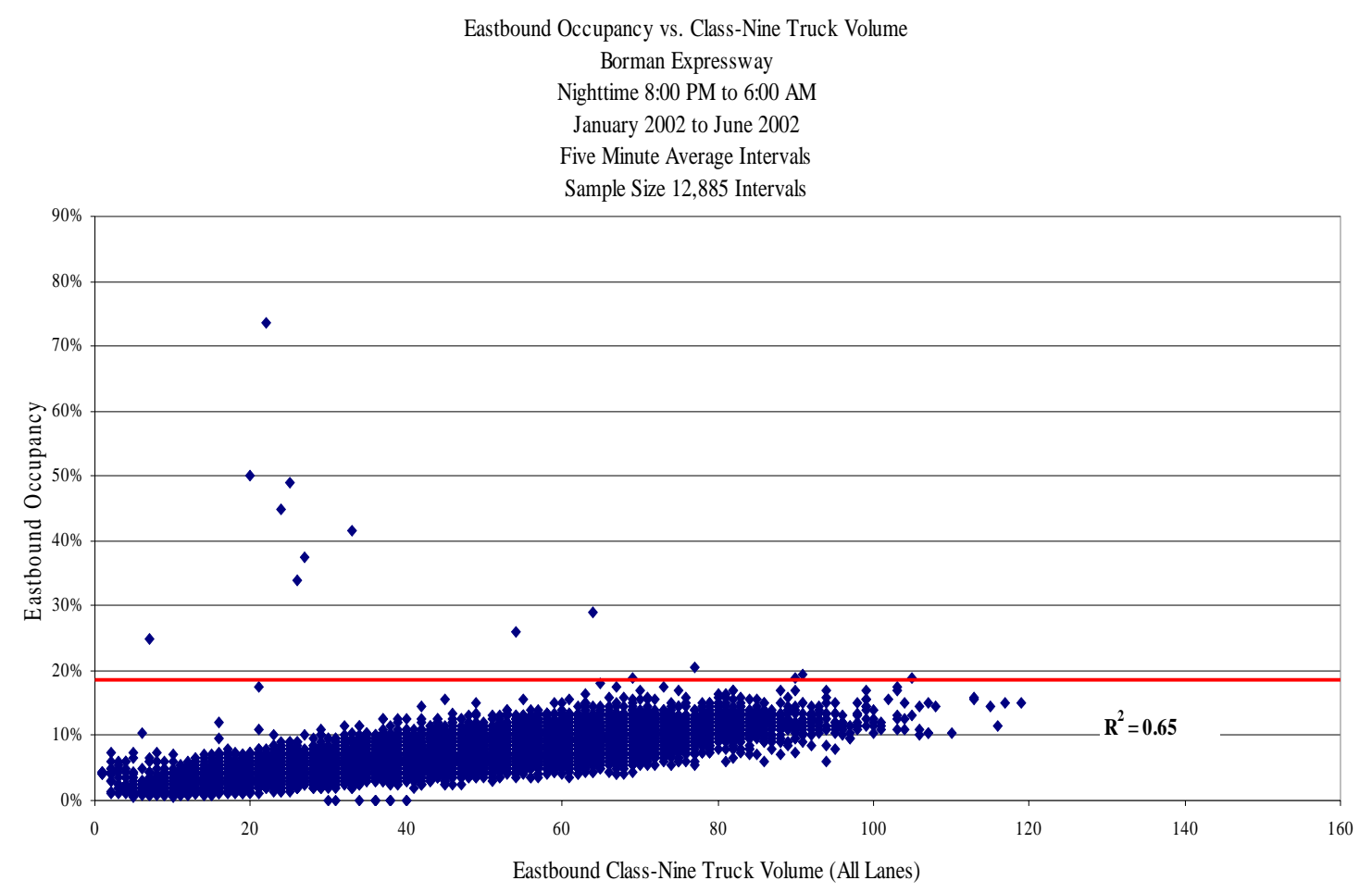

Figure 4.9: Nighttime Eastbound Occupancy vs. Class-Nine Truck Volumes.

\subsubsection{Nighttime Westbound Occupancy vs. Class-Nine Truck Volume}

The westbound occupancy vs. class-nine volumes is shown in Figure 4.10. Both the Xaxis and the Y-axis are the same as the eastbound direction. Again, the same reasonably good 
correlation $\left(\mathrm{R}^{2}=0.72\right)$ between the occupancies and the volumes is shown when occupancies remain below $18 \%$. In addition to this trend, the $\mathrm{R}^{2}$ between the daytime and nighttime correlation are similar, with daytime of 0.72 and nighttime of 0.73 . If the entire dataset is used, the $\mathrm{R}^{2}$ changes from 0.06 during the daytime hours of operation to 0.54 during the nighttime.

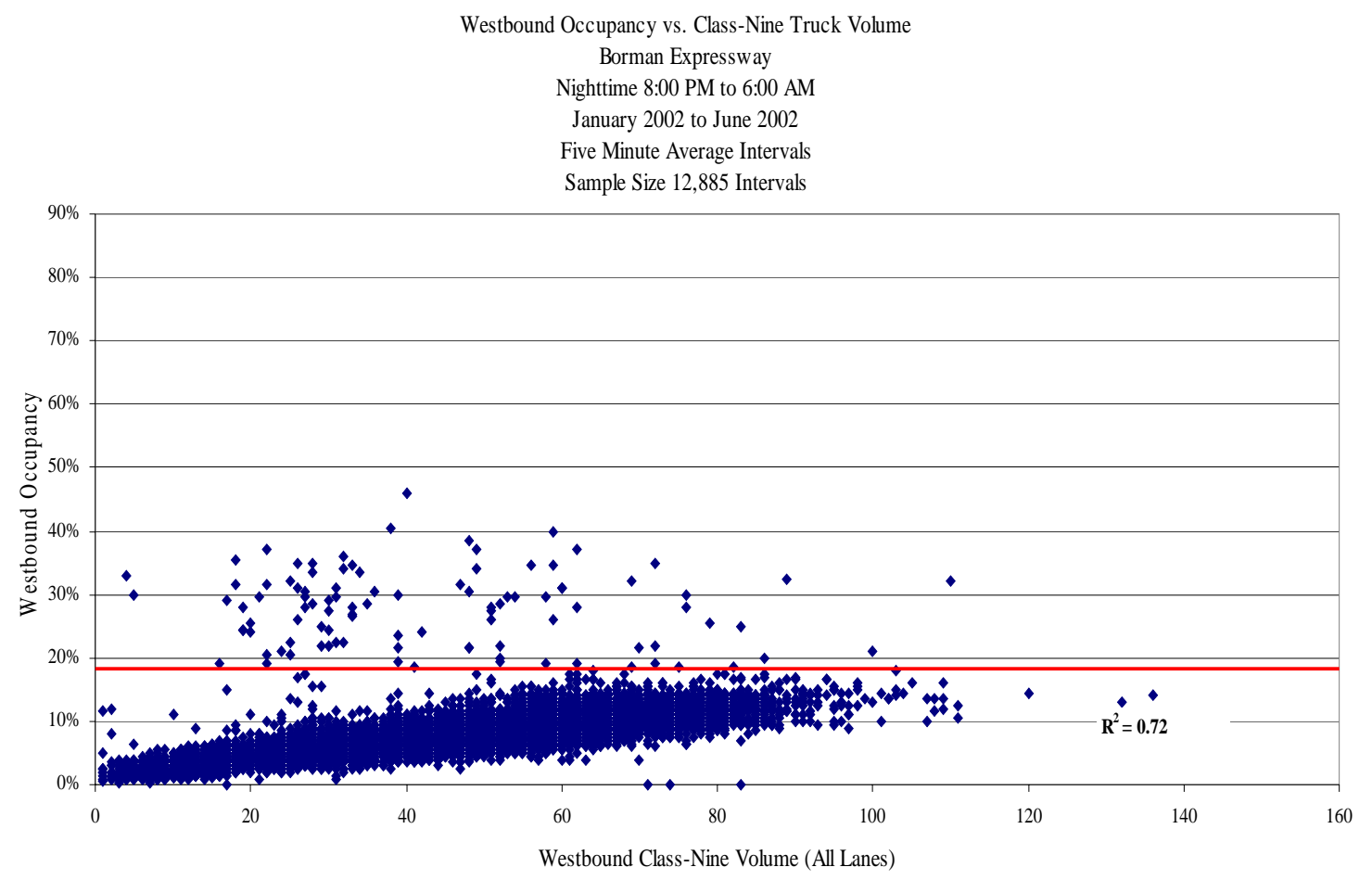

Figure 4.10: Nighttime Westbound Occupancy vs. Class-Nine Truck Volume.

The lower volumes traveling during the evening explain the large increase in the $\mathrm{R}^{2}$. Class-nine volumes decrease by 12 vehicles per five-minutes and car volumes decrease from 2,331 cars per hour to 912 cars per hour at night. The traffic demand at night is much less; therefore, the amount of congestion decreases from 2,806 intervals greater than 18\% to 99 intervals greater than $18 \%$ at night. For both nighttime directions, the westbound lanes continue to have higher periods of congestion, showing the impact of traffic patterns traveling towards the city of Chicago.

\subsubsection{Nighttime Eastbound Occupancy vs. Eastbound Speed}

Figure 4.11, eastbound occupancy vs. eastbound speed, highlights the difference between daytime and nighttime conditions along the Borman Expressway. In Figure 4.11 the scatter plot includes a majority of group one individual records $(12,745$ of 12,885$)$ with speeds greater or 
equal to $50 \mathrm{MPH}$ and occupancies less than or equal to $18 \%$. There are 98 points less than or equal to $45 \mathrm{MPH}$, and 13 of the 98 individual records have speeds less than $45 \mathrm{MPH}$ and occupancies greater than $10 \%$. This is a change from the 696 group four records during the daytime to the 13 records at night. The lower speeds with lower occupancies in this case are the result of trucks traveling slower at nighttime. Unlike the daytime hours, the traffic demand is lower; consequently, there is less congestion. In this instance, the lower average speeds reflect the personal preference of the truck driver and is not a by-product of congestion.

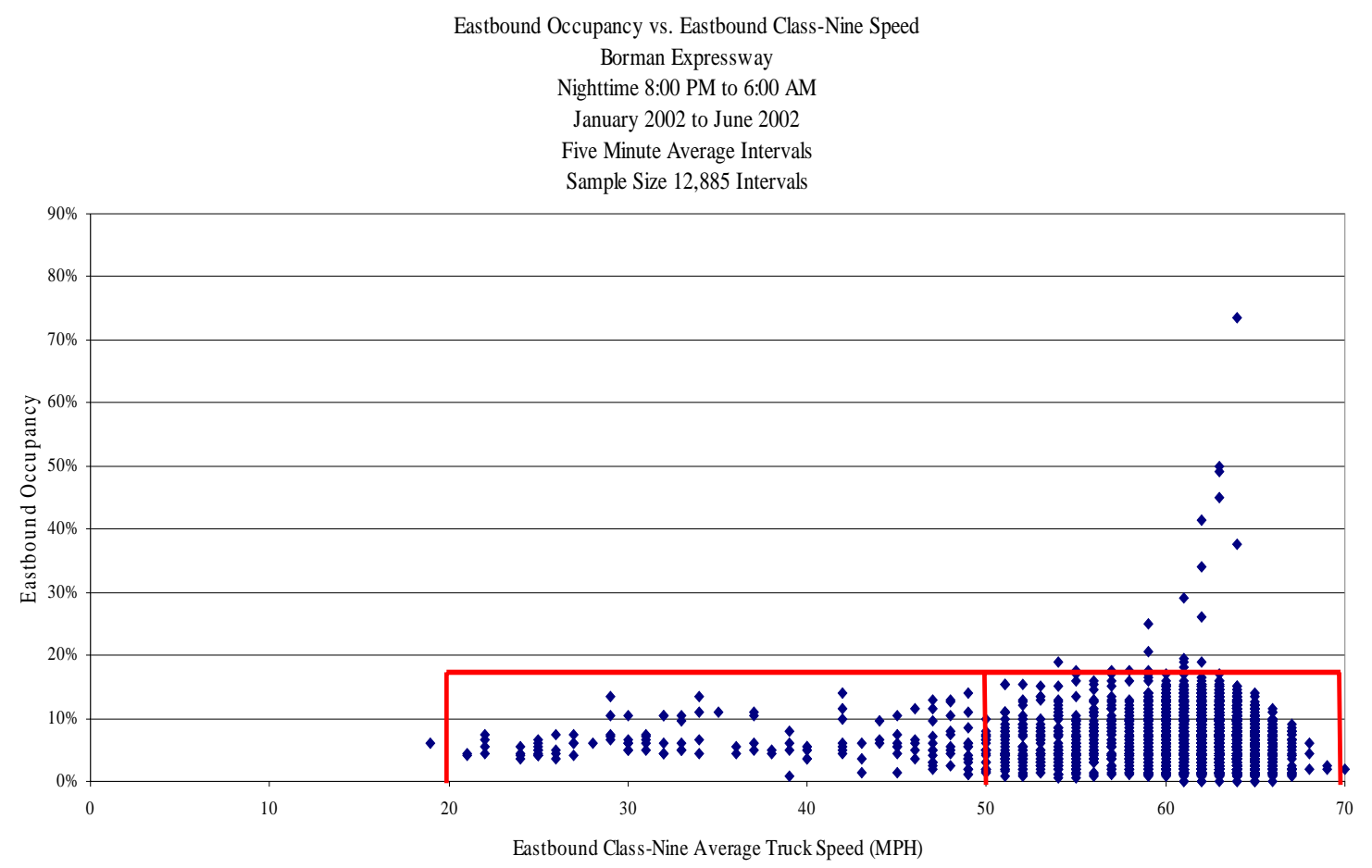

Figure 4.11: Nighttime Eastbound Occupancy vs. Eastbound Class-Nine Truck Speed.

Compared to daytime hours, the congestion period at night is very small and is mainly used to compare the possible differences between nighttime conditions. In terms of the model, there is very little congestion during the nighttime hours so a different congestion phase at night is selected, which is defined by speeds less than $50 \mathrm{MPH}$ and includes 140 records with occurrences less than $18 \%$. It is unlikely to have high levels of degradation on ambient air quality during this period. 


\subsubsection{Nighttime Westbound Occupancy vs. Westbound Speed}

The final scatter plot, Figure 4.12, is the nighttime westbound occupancy vs. the nighttime class-nine truck speed. Figure 4.12 has the same X-axis and Y-axis as Figure 4.11 and the same time duration, between 8:00 PM and 6:00 AM. To stay consistent with the eastbound nighttime boundary conditions for the nighttime analysis, the westbound is divided into two different groups, with normal traffic operations greater than or equal to $50 \mathrm{MPH}$ and occupancies less than or equal to $18 \%$, and more heavily congested areas with speeds less than $50 \mathrm{MPH}$.

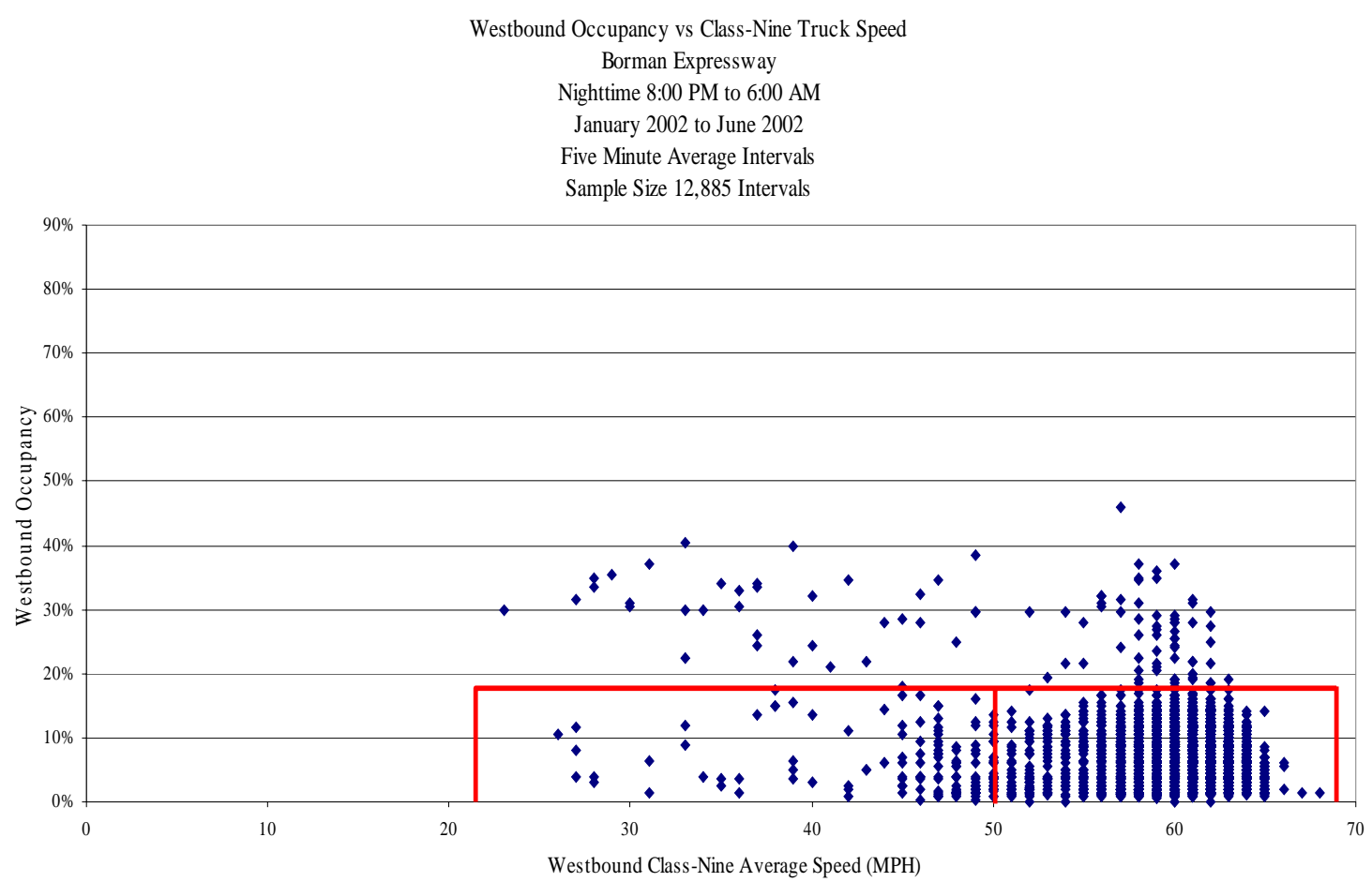

Figure 4.12: Nighttime Westbound Occupancy vs. Westbound Class-Nine Truck Speed.

As seen with the daytime comparisons, the nighttime comparisons are similar, with the westbound lanes having a larger amount of congestion in comparison to the eastbound direction. Westbound has 43 individual records with group four boundary conditions, which is an increase of 30 over the eastbound direction. The same rationale for the daytime differences may be used here also, i.e., traffic is dictated by the impact of the Chicago area and the traffic flow traveling towards the area with the bottleneck. Although there are still some areas of congestion, the 
congestion is significantly less, with a decrease from 1,098 to less than 43 records when compared to westbound daytime operations.

Within Figure 4.12, there are two different right lane closures, one on 1/31/02 at 2:00 AM EST and the second on 3/3/2002 at 3:00 AM EST. These two closures accounted for all but two individual intervals where speeds are less than $45 \mathrm{MPH}$ and the occupancies are less than or equal to $18 \%$. These two closures provide an excellent example of the impact of a lane closure at night. The average truck volume during this period is six trucks per five-minute interval. The traffic demand during this time is low and ultimately the speeds decreased because the trucks are traveling through a work zone; however, the occupancies do not increase since the capacity along the Borman Expressway with a lane closed is still greater than that required by the traffic demand during the early morning hours.

\subsection{Summary of Cluster Analysis}

The traffic characteristics between the eastbound and westbound directions are different between each other and the time of day. The worst congestion, or scattering of individual records, is graphically illustrated in Figures 4.4 and 4.6 for the westbound lanes during the daytime. The eastbound lanes during the daytime hours have the second highest amount of scattering or congestion areas.

The nighttime hours have the same trend, with the westbound lanes having higher congestion periods than the eastbound lanes. However, because of the lower traffic demand, the nighttime does not experience the same levels of congestion that are seen during the daytime hours. Because of different environmental and traffic conditions that occur between daytime and nighttime hours, the nighttime period is separated from the daytime period and two different models are derived in Chapter 5.

In summary, this chapter identified different cluster groups that are used in Chapter 5 in

order to better understand the cumulative effects of different traffic combinations from the Borman Expressway on local ambient air quality. 


\section{BORMAN-PM ${ }_{2.5}$ CLASIFICATION TREE}

\subsection{Daytime Combination of Different Groups}

Chapter 4 provided a methodology for determining the different boundary conditions or groups that are used in a classification tree model building process. As demonstrated in Chapter 4, the classification tree is divided into daytime and nighttime periods. Daytime includes four groups in the westbound direction. Group one includes normal operations with occupancies less than or equal to $18 \%$ and speeds greater or equal to $50 \mathrm{MPH}$. Group two has occupancies greater than $18 \%$ and less than or equal to $30 \%$ with speeds remaining greater or equal to $50 \mathrm{MPH}$. Group three has occupancies greater than $30 \%$ with speeds greater than or equal to $50 \mathrm{MPH}$, and group four is considered a breakdown period with speeds less than or equal to $45 \mathrm{MPH}$ and occupancies greater or equal to $10 \%$. The eastbound daytime lanes contain two different groups, group one and group four. Both of these groups have the same boundary conditions as westbound group one and group four.

\subsection{Daytime Definition of Operational Levels Along the Borman Expressway}

The two eastbound groups and four westbound groups, in combination with each other, produce the eight selected daytime combinations of traffic conditions along the Borman Expressway. Table 5.1 shows the eight different combinations of traffic conditions that are correlated with ambient $\mathrm{PM}_{2.5}$ flux levels. Operational level one shows both eastbound and westbound lanes traveling under normal conditions, which includes low occupancies and normal speeds, allowing the Borman Expressway to remain stable. The stable, efficient travel places lower stress on an engine and creates lower levels of emissions per vehicle and thereby creating higher levels or better ambient air quality.

Operational level two maintains normal conditions eastbound and increases the westbound total occupancy, changing the westbound direction from group one to group two. The group two westbound lanes are more unstable than group one westbound lanes, so it seems likely that the overall levels of ambient $\mathrm{PM}_{2.5}$ flux for operational level two when compared to operational level one will be higher in ambient $\mathrm{PM}_{2.5}$ flux. 
Table 5.1: Eastbound and Westbound Daytime Operational Levels

\begin{tabular}{|l|l|l|}
\hline Eastbound Direction & Westbound Direction & Operational Level \\
\hline Group One & Group One & One \\
\hline Group One & Group Two & Two \\
\hline Group One & Group Three & Three \\
\hline Group One & Group Four & Four \\
\hline Group Four & Group One & Five \\
\hline Group Four & Group Two & Six \\
\hline Group Four & Group Three & Seven \\
\hline Group Four & Group Four & Eight \\
\hline
\end{tabular}

Operational level three includes normal group one levels eastbound and group three levels westbound, with an increase in westbound occupancy to levels higher than $30 \%$. With the high levels of westbound occupancy, the westbound direction is considered to be a transitional phase and is highly unstable when directly compared to westbound group one and westbound group two. This unstable period in the westbound direction will create higher levels of ambient $\mathrm{PM}_{2.5}$ flux when directly compared with operational levels one and two.

Operational level four represents normal eastbound conditions, while the westbound direction experiences heavy congestion, or group four. During this period, the speeds westbound are less than $45 \mathrm{MPH}$ and the Expressway becomes a very inefficient link. This inefficiency creates the highest engine loadings per vehicle in the westbound direction and creates ambient $\mathrm{PM}_{2.5}$ flux levels higher than operational levels one, two, or three (Metz et al. 1997, Yanowitz et al. 2002).

Operational level five changes the eastbound direction from normal, group one, to congestion, group four. This is the first level where the eastbound direction experiences inefficient travels causing the engine loading in the eastbound direction to theoretically dominate the emissions on the Expressway (Metz et al. 1997, Yanowitz et al. 2002). While the eastbound direction experiences heavy congestion, the westbound direction remains under normal or group one condition.

Operational level six demonstrates heavy congestion in the eastbound lanes, while the westbound direction increases the total occupancy from a normal or group one condition to a group two condition. Operational level six should have a higher level of ambient $\mathrm{PM}_{2.5}$ flux than operational five due to the increased level of occupancies in the westbound level. Operational 
level seven continues this trend: with westbound levels of occupancy increases to greater than 30\%. Finally, operational level eight is the worst-case scenario for the Borman Expressway, with heavy congestion in both eastbound and westbound directions.

Theoretically, operation level one should produce the best air quality followed by levels two and three. Operational levels four and five should be similar, considering one lane direction is operating under normal conditions, while heavy congestion occurs in the other lane direction. It is possible that level four might be greater than level five due to the impact of the area surrounding Chicago. Levels six and seven continue to yield degradation in air quality in consort with the overall degradation in traffic conditions. Level eight is the worst-case scenario and is avoided: not only are the traffic conditions poor but the air quality is at its worst.

\subsection{Impact of Different Operational Levels on the Borman Expressway}

To validate this theory of degradation in air quality with respect to different operational levels along the Borman Expressway, traffic and environmental data are merged together. Because of the location of the ambient sampler with respect to the Expressway, only winds between 270 and 110 are selected. The heavy reliance on wind direction from the north decreases the traffic dataset from 30,624 individual records to 11,165 individual records. This reduced dataset is used to generate the frequency of occurrence for the different operational levels when wind direction transports the pollutants to the air monitor, and the graphical results are shown in Figure 5.1. Figure 5.1 shows that operational level one dominates the frequency of occurrence at $80.2 \%$. Operational levels two, three, and four each occur about $4 \%$ of the time, while operational levels six and seven almost never occur (less than $0.4 \%$ ). Operational level eight, the worst-case scenario, occurs only $1.5 \%$ of the time.

Due to the reliance of winds from the north (because of the location of the air monitoring station being on the south side of the Expressway), it is important to compare the frequency of occurrence between the traffic and environmental dataset. (The traffic dataset has no filtration based on wind direction.) In Table 5.2, the comparison between the traffic and environmental dataset and the total traffic dataset show the same overall trends: operational level one is the highest at $80.2 \%$ and $81.3 \%$, and the lowest frequency for occurrence are operational levels six and seven (less than $0.2 \%$ ). 


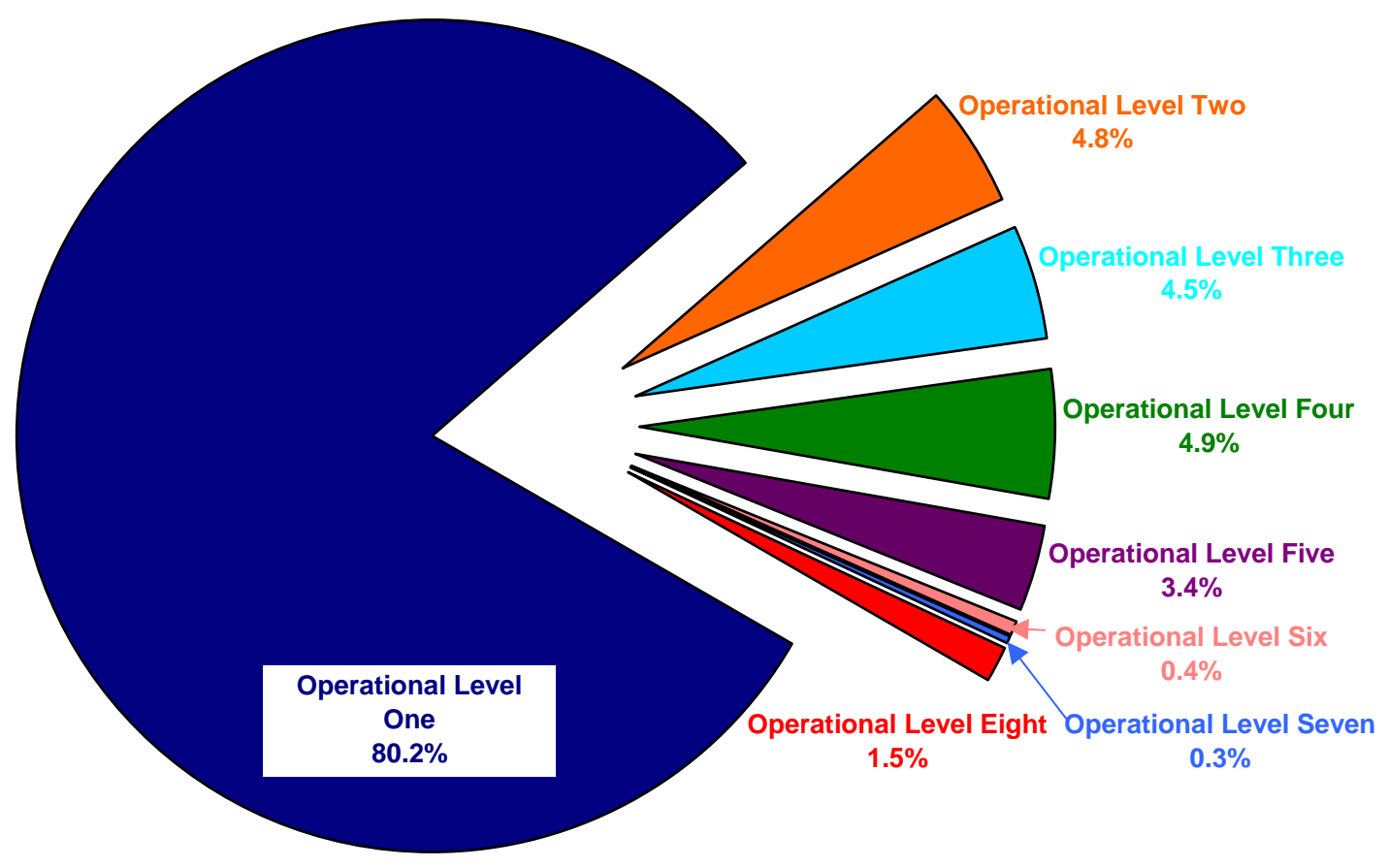

Figure 5.1: Daytime Frequency of Occurrence for Different Operational Levels

Without an appropriate sample size, operational levels six and seven may skew the final results. In both datasets, with sampling from January 2002 to June 2002, operational levels six and seven do not have a high frequency for occurrence and therefore are not considered in the final analysis.

Table 5.2: Daytime Frequency of Occurrence Between Traffic and Environmental Dataset and Total Traffic Dataset

\begin{tabular}{|l|l|l|}
\hline Operational Level & Traffic and Environmental Dataset & Total Traffic Dataset \\
\hline One & $80.2 \%$ & $81.3 \%$ \\
\hline Two & $4.8 \%$ & $4.6 \%$ \\
\hline Three & $4.5 \%$ & $5.1 \%$ \\
\hline Four & $4.9 \%$ & $4.8 \%$ \\
\hline Five & $3.4 \%$ & $2.5 \%$ \\
\hline Six & $0.4 \%$ & $0.2 \%$ \\
\hline Seven & $0.3 \%$ & $0.2 \%$ \\
\hline Eight & $1.5 \%$ & $1.2 \%$ \\
\hline
\end{tabular}


Overall, there are 17,431 records; operational level six and seven respectively combine for 36 and 43 records, combining for less than eight total hours.

\subsubsection{Daytime ANOVA for the Six Operational Levels}

The ANOVA is seen in Table 5.3 calculates the average $\mathrm{PM}_{2.5}$ flux values and compares these six different preliminary averages in order to determine the level of significance that is associated between all operational levels. The preliminary statistical significance between ambient $\mathrm{PM}_{2.5}$ flux and traffic operational levels; is represented in the ANOVA $\mathrm{i} / \mathrm{j}$ matrix. Operational level one has a $\mathrm{PM}_{2.5}$ flux level of 27.94 (ug/ $\mathrm{m}^{2}$ sec), operational level two has $\mathrm{PM}_{2.5}$ flux of 32.06 (ug/ $\mathrm{m}^{2 *} \mathrm{sec}$ ), level three is $33.50\left(\mathrm{ug} / \mathrm{m}^{2} * \mathrm{sec}\right)$ and level four is $36.74\left(\mathrm{ug} / \mathrm{m}^{2 *} \mathrm{sec}\right)$. Levels two, three, and four, in comparison to level one, all have a p-value $<0.001$ and a reasonably good correlation $\mathrm{R}^{2}$ of 0.97 between the average $\mathrm{PM}_{2.5}$ flux levels for operational levels one through four.

Table 5.3: ANOVA Table for Daytime Correlation between Operational Levels and Ambient $\mathrm{PM}_{2.5}$ Flux.

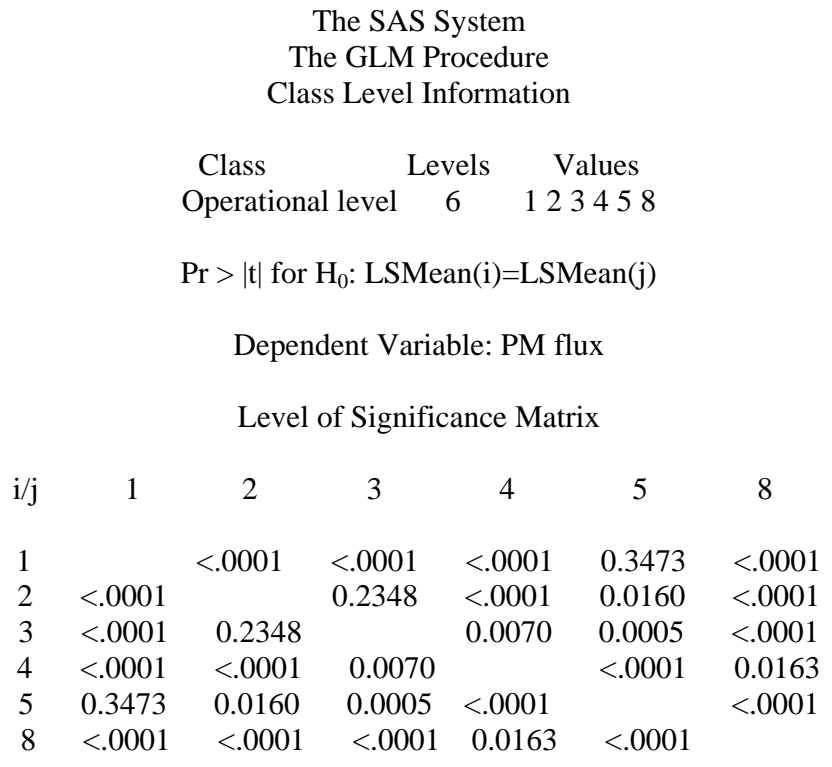

Operational level four has the second highest value and remains consistent, having the worst congestion level on the westbound side. This supports the theory that the highest engine loadings occur during periods with the highest congestion (Metz et al. 1997, Yanowitz et al. 2002). When eastbound lanes are operating normally and the westbound lanes change from operational level one to operational level four, the air quality decreases by $31.5 \%$. 
Operational level two, in comparison to level three, is not significantly different (p-value $<0.234)$. This marginal significance suggests that when traffic remains greater or equal to 50 $\mathrm{MPH}$, the increase in westbound occupancy does not significantly change the overall levels of $\mathrm{PM}_{2.5}$ flux. The truck volumes do not change significantly so the increase in occupancy is related to the increase in the volume of cars that are not a major source for $\mathrm{PM}_{2.5}$. For the final model, operational levels two and three are combined for both because there is little significant difference.

Operational level five consists of normal operations in the westbound direction and heavy congestion along the eastbound direction. Operational level five has a p-value of 0.35 in comparison with operational level one. This was surprising and suggests that 1) degradation from traffic leaving the city of Chicago traveling in the eastbound direction does not significantly impact the $\mathrm{PM}_{2.5}$ ambient air quality; and 2) there is less chance of bottlenecking in this direction. Based on this information and considering that INDOT has a limited amount of funding, it is better to focus financial efforts on the westbound lanes, where there is the greatest opportunity to improve ambient $\mathrm{PM}_{2.5}$ air quality.

With a value of $40.9 \mathrm{PM}_{2.5}$ flux (ug/m² ${ }^{2} \mathrm{sec}$ ), operational level eight, which occurs $1.5 \%$ of the total time, is the worst case scenario in terms of poor air quality (46.5\% higher than operational level one). Finally, with low average speeds, volumes, high occupancies, and poor air quality, there are no positive attributes in allowing the Expressway to operate at level eight and this level should be avoided altogether.

\subsection{Nighttime Combination of Different Groups}

For both directions, there are two different groups explained in Chapter 4: the first group contains speeds greater or equal to $50 \mathrm{MPH}$ and occupancies less than or equal to $18 \%$ and the second group contained speeds less than $50 \mathrm{MPH}$. There are four different nighttime combinations or operational levels on the Expressway. These levels are shown Table 5.4.

Table 5.4. Nighttime Combination of Different Groups for Different Nighttime Operational Levels

\begin{tabular}{|l|l|l|}
\hline Operational Level & Eastbound Direction & Westbound Direction \\
\hline 9 & Normal & Normal \\
\hline 10 & Congestion & Normal \\
\hline 11 & Normal & Congestion \\
\hline 12 & Congestion & Congestion \\
\hline
\end{tabular}


The four operational levels include normal conditions for both eastbound and westbound directions seen in operational level nine; while operational level ten occurs during eastbound congestion while the westbound direction remains under normal conditions. Operational level eleven is the opposite of ten, with congestion occurring westbound and normal conditions in the eastbound direction. The final operational level, twelve, has congested periods for both lane directions.

The traffic dataset and the environmental datasets are merged together in a similar manner to the daytime hours and the frequency of occurrence with correct wind directions is shown in Figure 5.2.

Figure 5.2 shows the overwhelming occurrence of operational level nine, where both eastbound and westbound conditions are normal. The frequency of occurrence between the traffic and environmental dataset and the total traffic dataset are compared in Table 5.5.

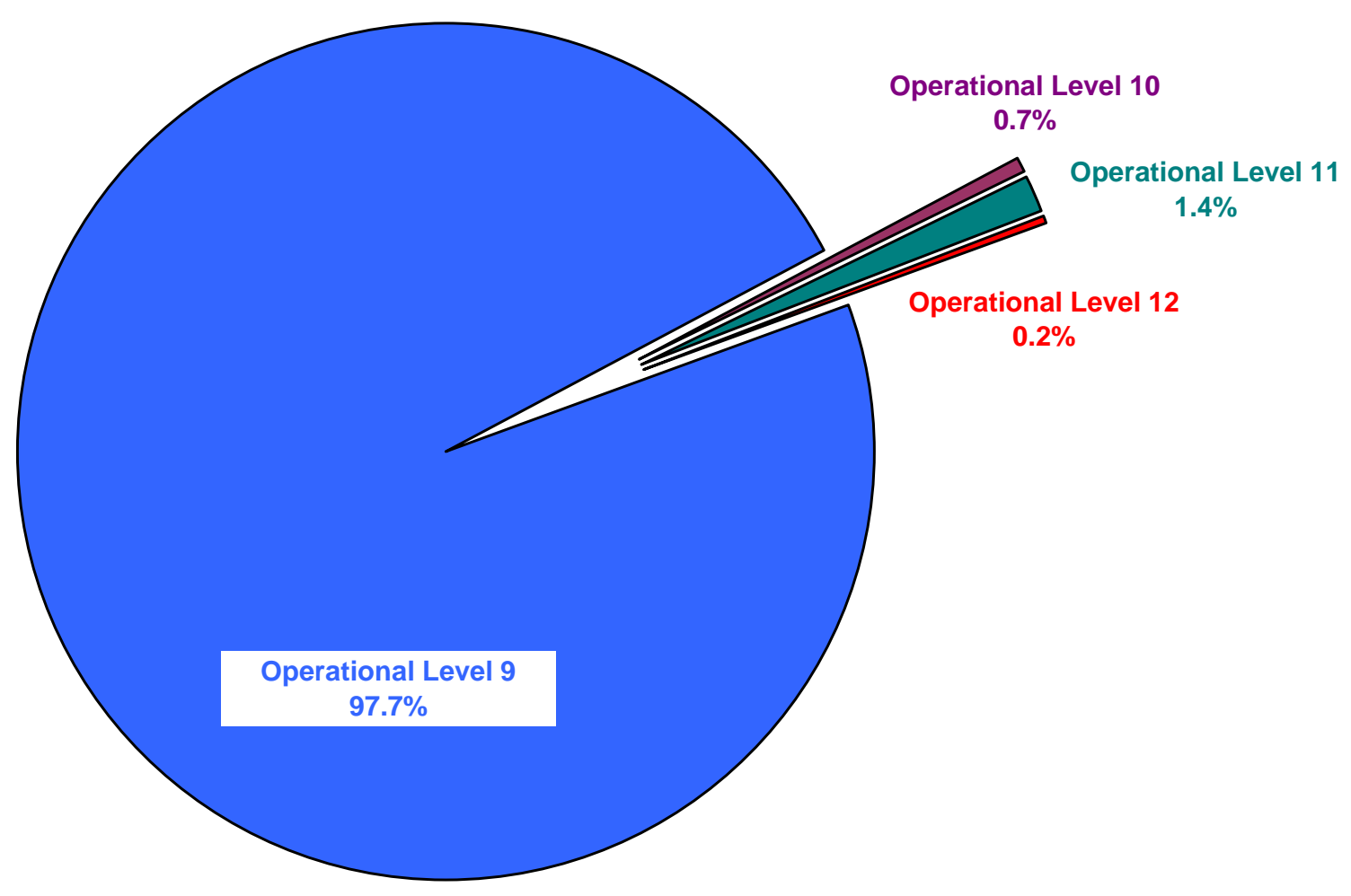

Figure 5.2: Nighttime Frequency of Occurrence for Different Operational Levels 
Table 5.5: Frequency of Occurrence between Nighttime Traffic and Environmental Dataset and Total Traffic Dataset

\begin{tabular}{|l|l|l|}
\hline Conditional Level & Traffic and Environmental Dataset & Traffic Dataset \\
\hline 9 & $97.7 \%$ & $98.4 \%$ \\
\hline 10 & $0.7 \%$ & $0.7 \%$ \\
\hline 11 & $1.4 \%$ & $0.8 \%$ \\
\hline 12 & $0.2 \%$ & $0.1 \%$ \\
\hline
\end{tabular}

The nighttime traffic conditions along the Borman Expressway are very free flowing, having few cases of congestion. There are 4,175 five-minute sample intervals comprised in Figure 5.2, 29 of which are operating at a level ten, and 10 in level twelve. Over the entire traffic dataset, level ten occurs 84 times, level eleven occurs 93 times, and level twelve occurred 11 times. Due to the sample size and the infrequent nature of operational levels ten, eleven, and twelve, they are not examined further.

When compared to the other operational levels, operational level nine is significantly different in all cases with a p-value $<0.0001$ with an improvement of $20.3 \%$ as compared to the normal daytime activity. The lower level of ambient $\mathrm{PM}_{2.5}$ flux is a direct result of the lower traffic demand over the course of the nighttime hours. The more constant speeds in conjunction with less traffic allow for lower levels of ambient $\mathrm{PM}_{2.5}$ flux, which means that this is the best time to travel the Expressway from traffic and air quality perspectives.

\subsection{Eight Descriptive Factors for the Classification Tree}

With the preliminary model validating the statistical difference between different operational levels, a final, more versatile descriptive model is derived. This final model includes one more factor or subgroup, class-nine truck volumes. For example, the final descriptive model, Figure 5.3, is a classification tree model that is based on the grouping or cluster analysis in Chapter 4 that is used for the purpose of identifying the aggregate boundary conditions that produces the highest statistically significant clusters. The entire daytime and nighttime traffic and environmental dataset is used to create this model. The classification tree is capable of describing the impact on ambient $\mathrm{PM}_{2.5}$ flux adjacent to the Borman Expressway based on different levels of traffic activity. The model is unable to describe events that occur outside the sampling boundaries shown earlier in Chapters 4 and 5, for example, one case might have high levels of congestion 
during nighttime hours. There are eight different factors used by the classification tree to identify the level of traffic activity and the Borman's influence on local ambient air $\mathrm{PM}_{2.5}$ flux. To elucidate the descriptive model shown in Figure 5.3, each of the eight factors are explained one at a time in the following sections.

These factors are:

1. Time of Day

2. Westbound Class Nine Average Speed

3. Westbound Occupancy

4. Eastbound Class Nine Average Speed

5. Eastbound Occupancy

6. Eastbound Class Nine Truck Count

7. Westbound Class Nine Truck Count

8. The Total Eastbound and Westbound Counts 


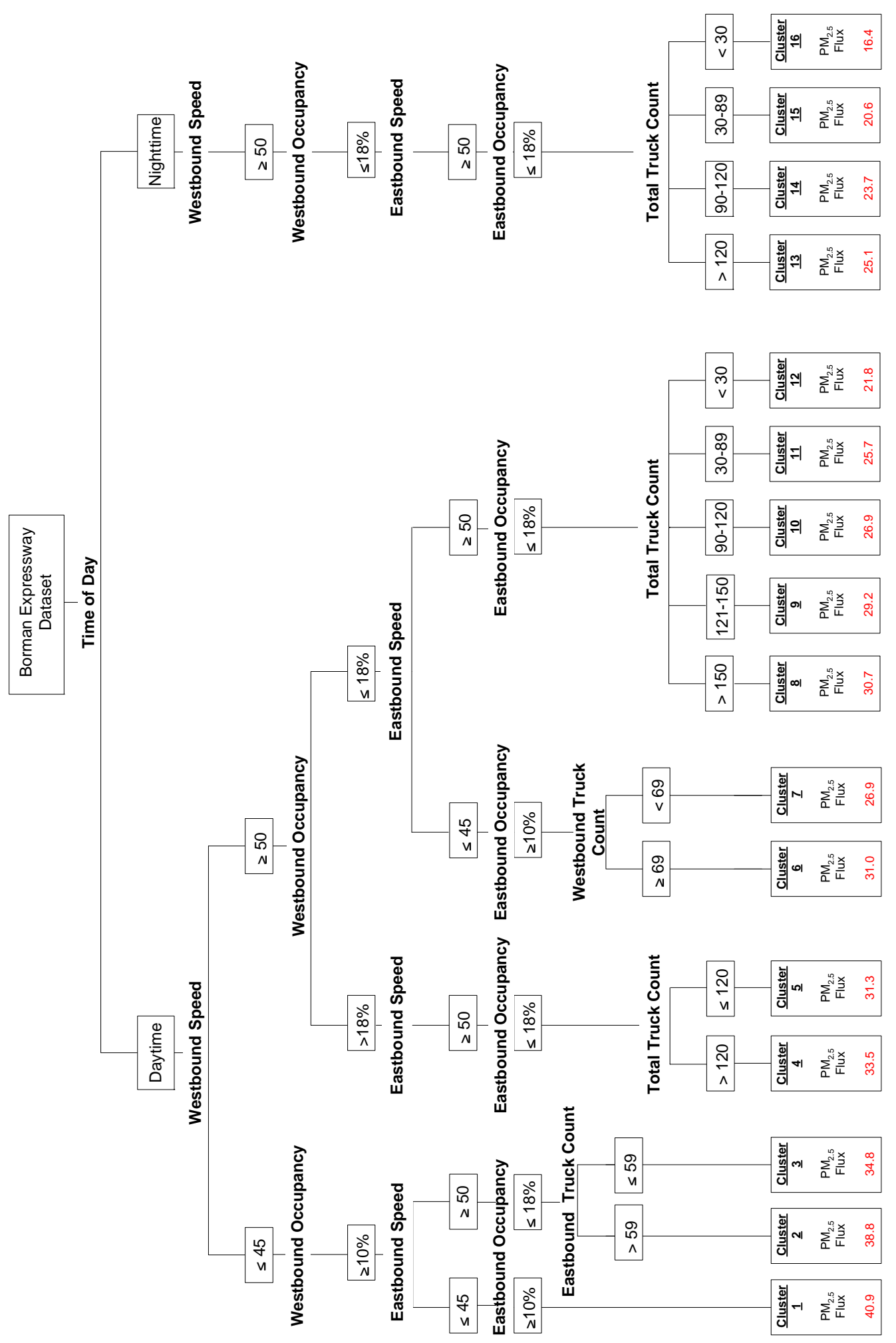

Figure 5.3: Classification Tree Model for Borman Expressway 


\subsection{The Classification Tree Model for Nighttime Conditions}

If nighttime hours are selected, the model moves to the right branch of the classification tree as shown in Figure 5.4.

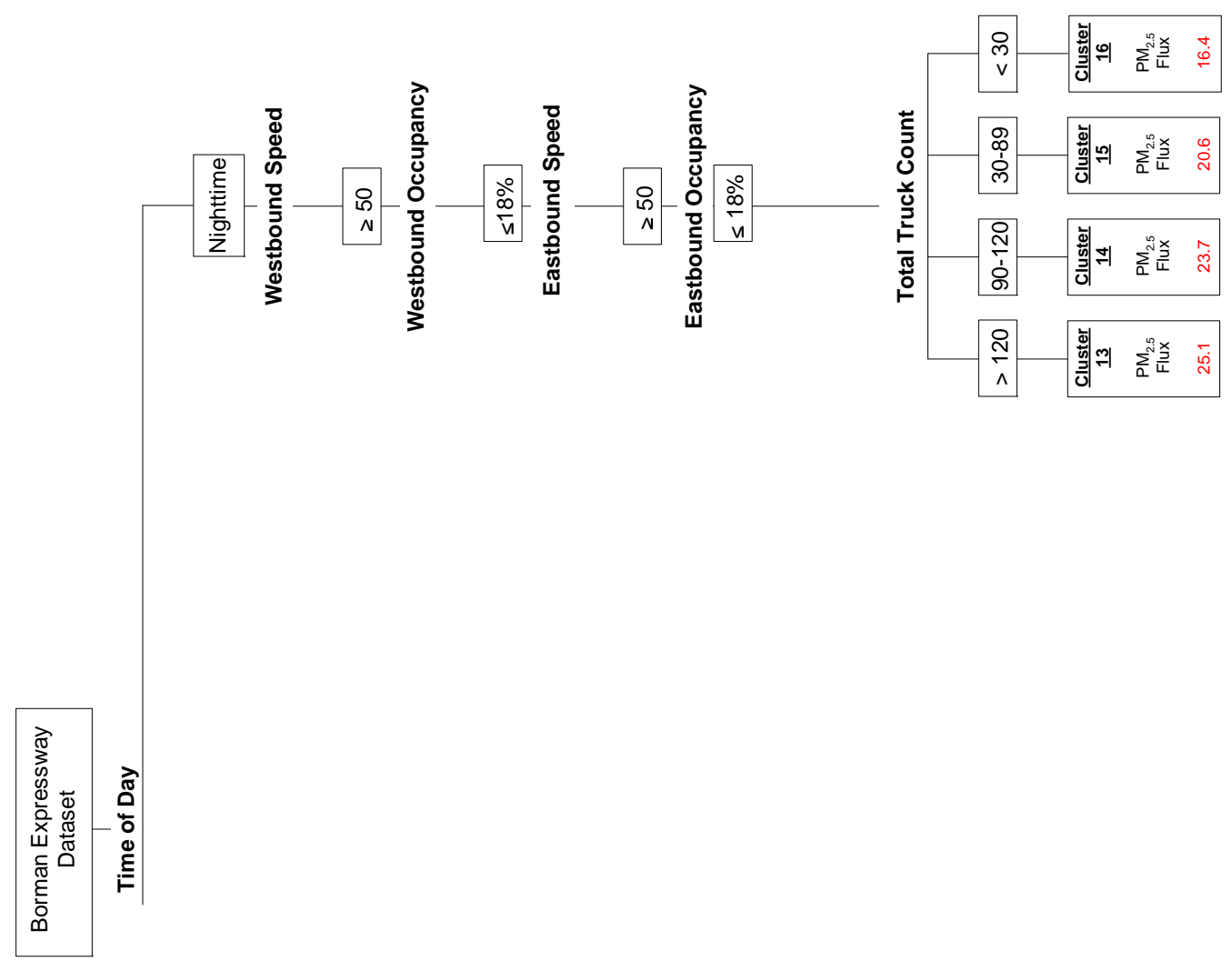

Figure 5.4: Nighttime Branches of Classification Tree Model

The next factor for the nighttime operation is westbound speed. In order to continue with evening hours, the westbound speeds must be greater or equal to $50 \mathrm{MPH}$. If the desired speed for nighttime operation is less than $50 \mathrm{MPH}$, the classification tree stops because speeds less than $50 \mathrm{MPH}$ violate the boundary conditions for this model. In order to continue with the nighttime branch the boundary conditions for each nighttime factor cannot be violated. The next factor concerns westbound occupancy, requiring occupancy less than or equal to $18 \%$, otherwise the selection is outside the boundary limits. Now that the overall westbound conditions are known, we turn to the eastbound traffic levels during the nighttime hours. The eastbound average speed 
must be greater than or equal to $50 \mathrm{MPH}$ and the eastbound occupancy must be less than or equal to $18 \%$.

The nighttime traffic conditions on the Borman Expressway are stable and the final model reflects this stability and does not allow for a wide variety of boundary conditions. These four boundary conditions may seem to limit the overall nighttime levels on the Expressway but it is important to note that these four boundary conditions allow $97.7 \%$ of the data from the traffic and environmental dataset and $98.4 \%$ of the total traffic dataset. These four boundary conditions determine the eastbound and westbound conditions during the nighttime hours and suggest normal operational levels during this time.

During this period of time, the engine loadings are the same for each truck so the higher volume of trucks should increase the overall levels of ambient $\mathrm{PM}_{2.5}$ flux. In this case, the total truck volume creates four different subgroups. The first subgroup includes total truck volume of less than 30 class-nine trucks. The second subgroup contains volumes greater or equal to 30 and less than 90 trucks. The third subgroup has volumes greater or equal to 90 and less than or equal to 120 , the final subgroup includes truck volumes greater than 120 class-nine trucks per fiveminute intervals. Class-nine volumes may be converted to total truck volumes by using Equations 3.1 and 3.2. Boundary conditions for these subgroups or clusters are selected to produce the greatest amount of classifications with the highest level of statistical significance. In this case clusters 13 and 14 have a p-value of less than 0.0233 , while the remaining clusters have p-values less than 0.001 . Figure 5.5 is a graphical representation of the change in clusters from the worst-case nighttime condition to the best-case nighttime condition. The X-axis reflects the different branches of the classification tree for the different nighttime conditions along the Borman Expressway. The left $\mathrm{Y}$-axis is ambient $\mathrm{PM}_{2.5}$ flux, while the right $\mathrm{Y}$-axis is the percent difference between clusters 13,14 ,and 15 , with respect to cluster 16 , which is the best-case scenario from an air quality standpoint.

The lower trend line is the ambient $\mathrm{PM}_{2.5}$ flux for the different conditions. This trend line shows the impact of increasing traffic volumes under normal operating conditions, with cluster 13 showing higher truck volumes greater than 120 correlate with the highest $\mathrm{PM}_{2.5}$ flux levels, while cluster 16 with the lowest truck volumes has the best air quality. The average $\mathrm{PM}_{2.5}$ flux levels change from 25.1 to 23.7 to 20.6 and $16.4\left(\mathrm{ug} / \mathrm{m}^{2} * \mathrm{sec}\right)$ respectively. The upper trend line is the percent difference between clusters 13,14 , and 15 with relation to cluster 16 . This trend shows that while traffic conditions remain normal, the ambient $\mathrm{PM}_{2.5}$ flux increases by more than $52.8 \%$. (Normal traffic conditions have speeds greater or equal to $50 \mathrm{MPH}$ and occupancies less than or 
equal to $18 \%$ ). The trend line also shows air quality improvements of $8.4 \%$ by changing from a cluster 13 to a cluster 14 . Air quality improves by $18.7 \%$ from cluster 14 to 15 . Finally, there is a $23.7 \%$ improvement from cluster 15 to cluster 16 . The air quality improvements show that while the Borman Expressway operates under normal conditions, the air quality near the Expressway may still be improved.

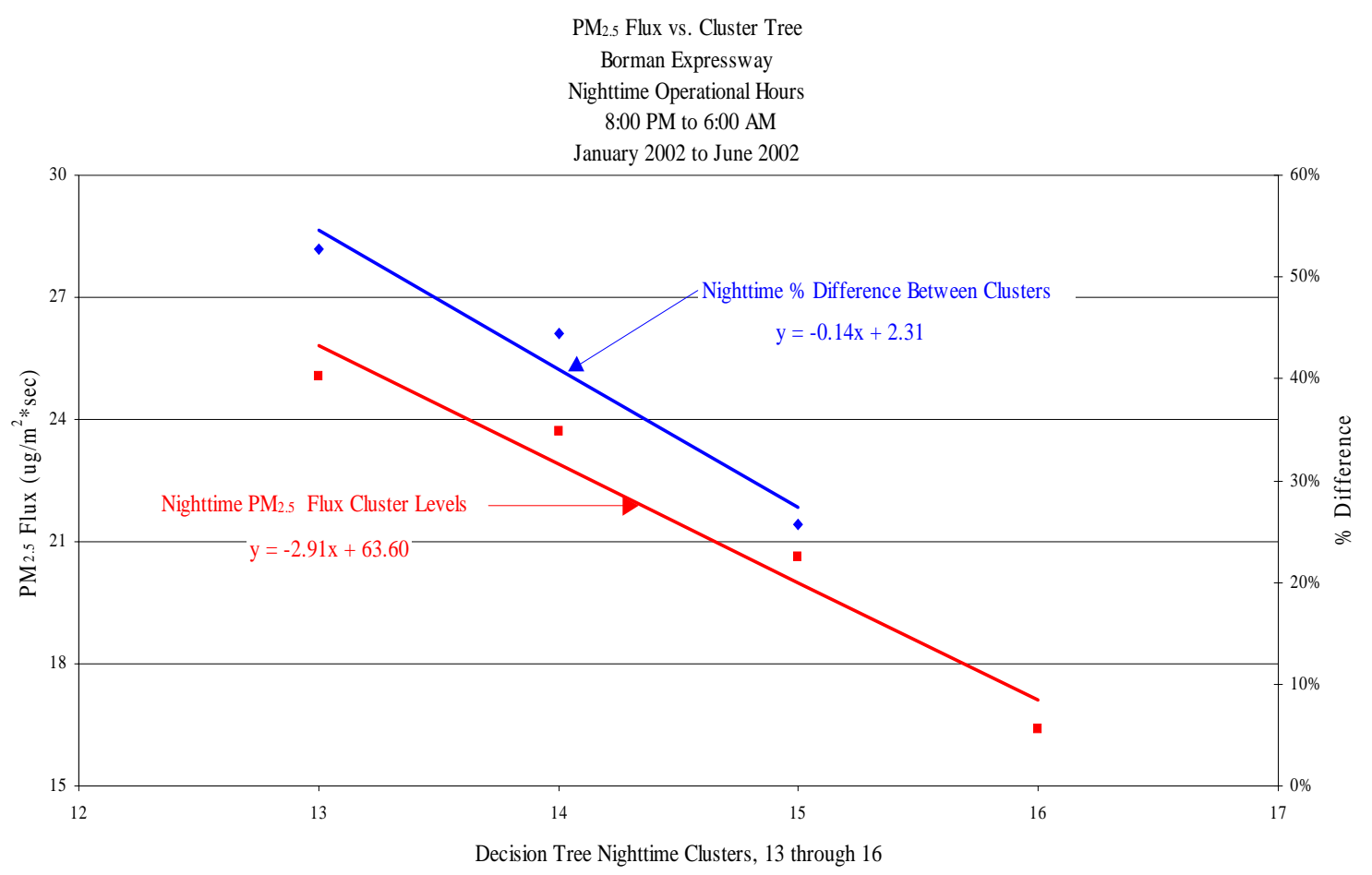

Figure 5.5: $\mathrm{PM}_{2.5}$ Flux vs. Nighttime Groups

\subsection{The Classification Tree Model for Daytime Conditions}

The evening hours consisted of one branch of the classification tree due to the overwhelming frequency of one operational level nine (97.7\%) occurring at nighttime. The daytime hours have more variability, allowing for more branches on the classification tree. The daytime hours will be divided into four different branches. The daytime branches of the classification tree are still comprised of the same group of factors explained in section 4.5.

\subsubsection{Classification Tree Daytime Branch One}

The first daytime branch, Figure 5.6, includes westbound speeds greater than or equal to $50 \mathrm{MPH}$, followed by westbound occupancies less than or equal to $18 \%$. These conditions classify the daytime westbound traffic as a normal condition. The eastbound remains greater or 
equal to $50 \mathrm{MPH}$, with occupancies less than or equal to $18 \%$. These boundary conditions indicate that the eastbound lanes exist under normal conditions. Since both the eastbound and the westbound conditions are normal, the total class-nine volumes are used to generate the different final branches or clusters on the classification tree. The final model selects five different traffic volumes for normal daytime operations: clusters 8, 9, 10, 11, and 12 .

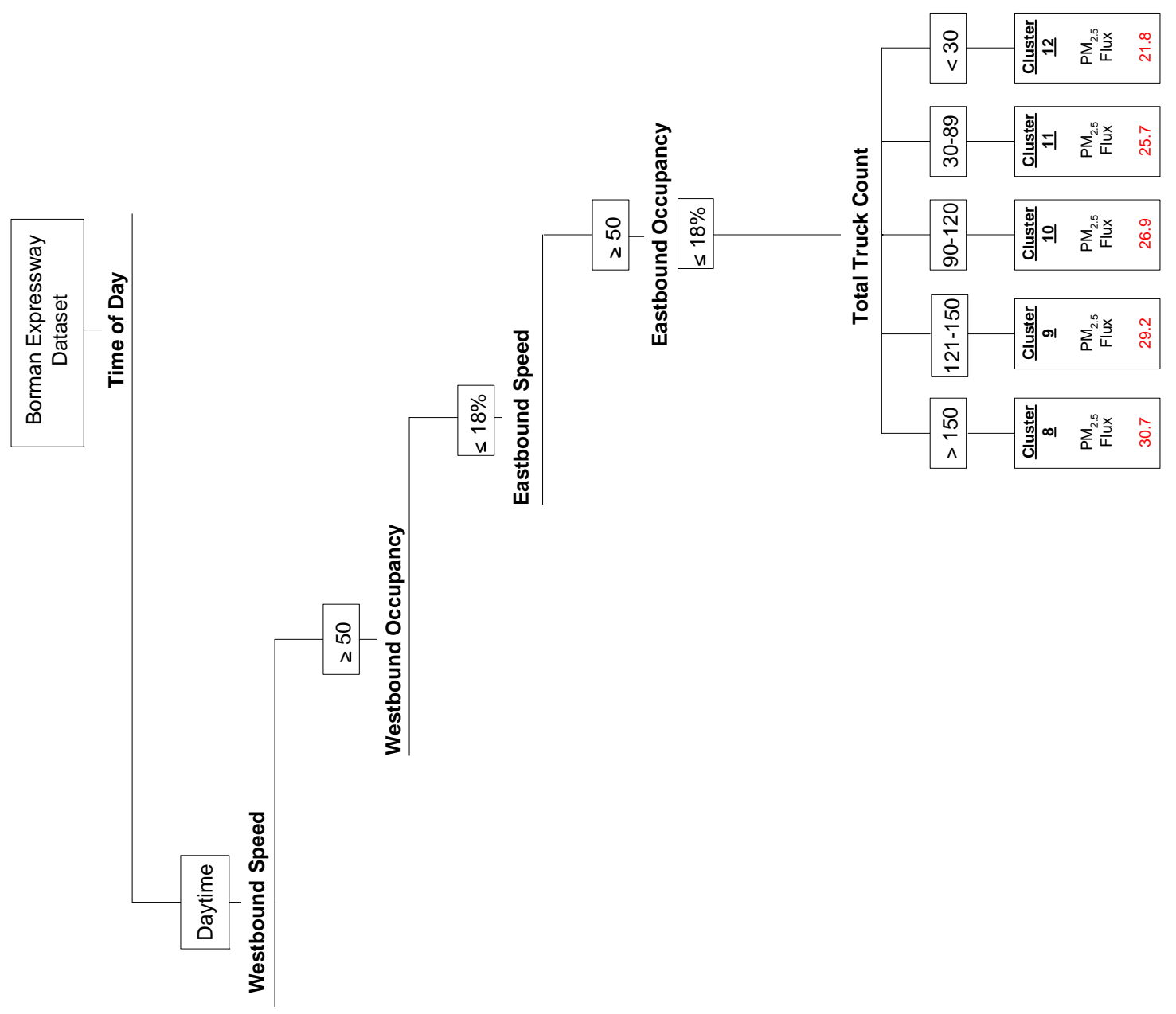

Figure 5.6: Classification Tree for Normal Daytime Operations

Boundary conditions in terms of traffic volumes are as follows: cluster 8 includes greater than 150 trucks, cluster 9 has greater than 120 trucks but less than or equal to 150 trucks, cluster 10 includes greater or equal to 90 trucks and less than or equal to 120 trucks, group eleven contains greater than or equal to 30 trucks and less than 90 trucks and the final cluster, cluster 12, has fewer than 30 trucks per five-minute intervals. Figure 5.7 illustrates the trend in ambient 
$\mathrm{PM}_{2.5}$ flux between the different normal conditions associated with clusters 8, 9, 10, 11, and 12 (upper trend line). The $\mathrm{X}$-axis is the different clusters from normal daytime conditions; the right $\mathrm{Y}$-axis is ambient $\mathrm{PM}_{2.5}$ flux levels, and the left $\mathrm{Y}$-axis is the percent difference between the different clusters with respect to cluster 12 .
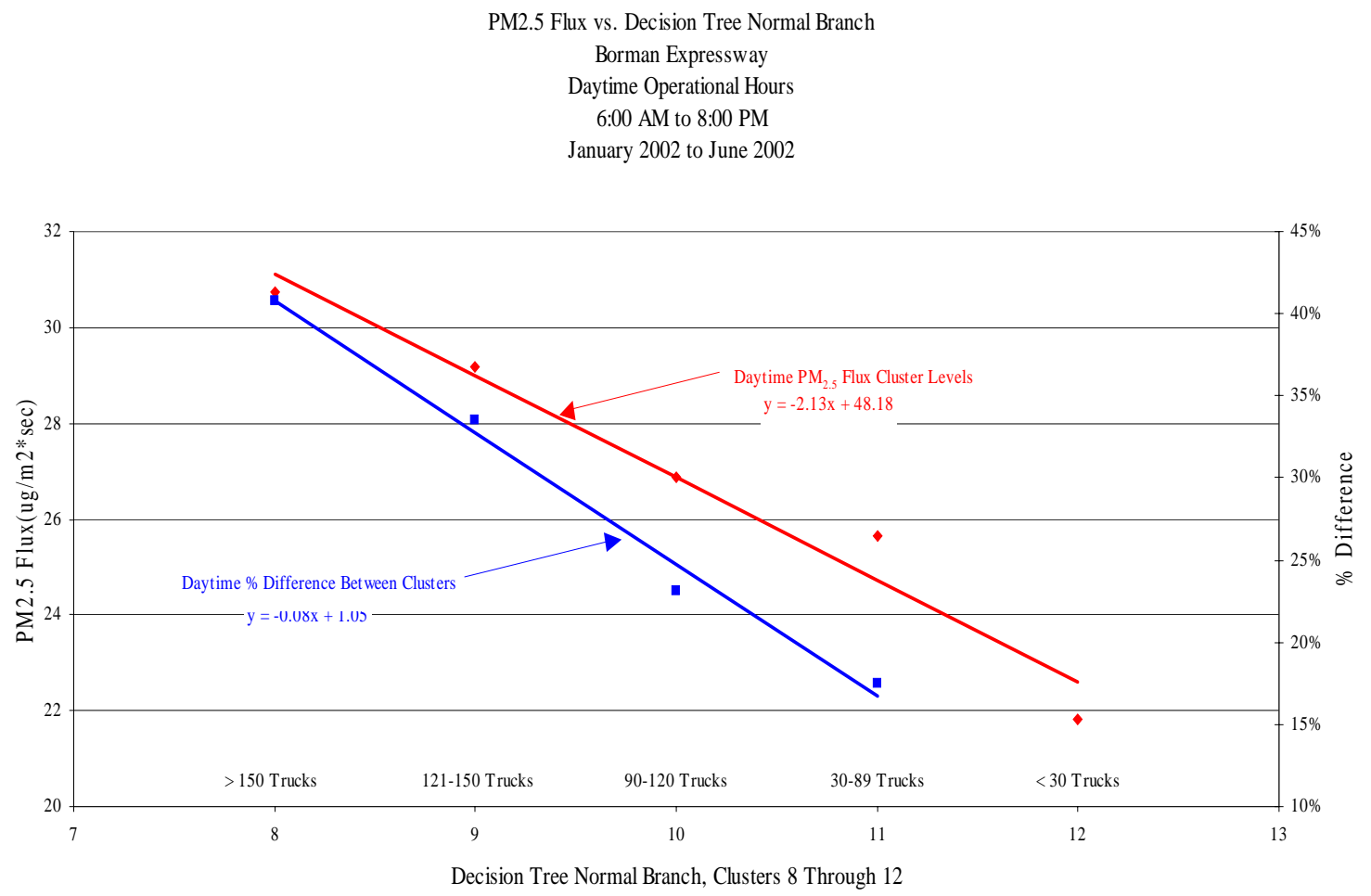

Figure 5.7: Daytime Classification Tree Branch for Normal Traffic Levels

As the volume of traffic increases throughout normal conditions, the ambient $\mathrm{PM}_{2.5}$ flux degrades. Under these conditions, even though the occupancy is low and the speeds are greater than or equal to $50 \mathrm{MPH}$, the Expressway will contribute significantly to different ambient $\mathrm{PM}_{2.5}$ flux levels. The ANOVA model for these five clusters show a level of significance with p-values less than 0.0314 (between clusters 10 and 11); the remaining p-values are all less than 0.003 . The ANOVA model clearly shows a significant difference between these five clusters.

The lower trend line is the percent difference between the best-case scenario, (cluster 12, normal conditions and low truck volumes), and the remaining four clusters: 8, 9, 10 and 11. A design tool for ITS shows that air quality changes by $40.8 \%$ when class-nine traffic volumes change from less than 30 trucks per five minutes to greater than 150 trucks per five minutes. Although it is unrealistic to run the Borman Expressway under low traffic volumes throughout the 
day, there are significant benefits. As high as $10 \%$ improvement in $\mathrm{PM}_{2.5}$ flux air quality may occur with the increase in one cluster level. So it may not be realistic to immediately change from 150 plus trucks to less than 30, but changing from 140 trucks down to 100 may be a possible and more realistic expectation.

\subsubsection{Comparison Between Normal Daytime and Nighttime Clusters}

Normal daytime cluster levels are also compared with nighttime cluster levels under the same conditions. Clusters 12 and 16 had a significance level of 0.0036 , clusters 11 and 15 had a significance level of $<0.001$, clusters 10 and 14 have a significance level of $<0.001$, and cluster 9 and 13 had a significance level of $<0.001$. In all cases, the daytime operating levels have higher ambient $\mathrm{PM}_{2.5}$ flux. This is explained by the increase in car volumes during the daytime hours as compared to the nighttime hours. Even though cars are not high emitters of particulate matter, they do change the conditions on the Expressway by creating higher demand levels, increasing the overall levels of ambient $\mathrm{PM}_{2.5}$ flux.

\subsubsection{Classification Tree Daytime Branch Two}

The next branch of the classification tree, Figure 5.8, demonstrates the westbound speeds remaining greater or equal to $50 \mathrm{MPH}$, with occupancies less than or equal to $18 \%$. When the eastbound direction becomes more heavily congested, the speeds decrease to less than or equal to $45 \mathrm{MPH}$, and the occupancy increases to greater than or equal to $10 \%$. Because of the difference in conditions on the Expressway the engine loadings per side will not be the same; thus, taking the total count will produce invalid results. There is a difference between 30 trucks traveling under heavy congestion and 30 trucks traveling under normal conditions. Therefore, the last branch in Figure 5.8 is divided into traffic counts on the opposite side from congestion. During congestion, the traffic flow is so unstable that the actual conditions are hard to measure. For example, there may be platoons that allow many trucks to pass quickly while during other periods very little volume is able to pass through.

Under normal traffic operating conditions, such as clusters 8 through 12, the change in volumes will have a statistically significant impact on air quality. In this branch, the westbound direction is operating under normal conditions and the change in volume in the westbound direction significantly impacts the air quality. The boundary condition for clusters 6 and 7 is 69 class-nine trucks traveling in the westbound direction, which is the average class-nine vehicle count under this set of conditions. Cluster 7 has less than 69 class-nine trucks, while cluster 6 has 69 or more class-nine trucks traveling westbound in a five-minute interval. Also it is desirable in this case to have both clusters possessing nearly the same sample size (111 for cluster 6, and 114 
for cluster 7). There is a $15.2 \%$ increase in ambient $\mathrm{PM}_{2.5}$ flux between clusters 6 and 7 with a pvalue $<0.0284$.

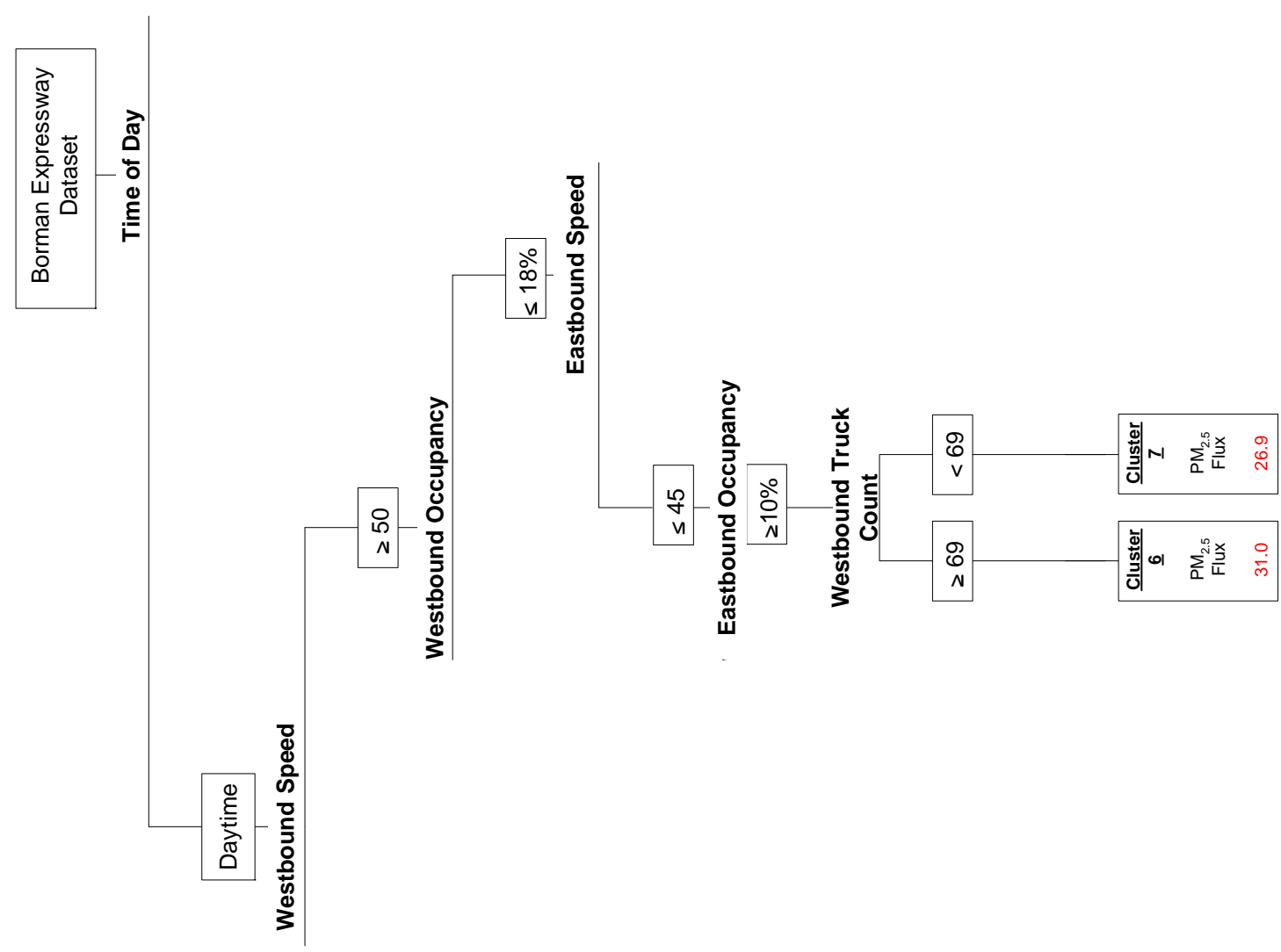

Figure 5.8: Daytime Classification Tree For Congestion Eastbound

Unlike the preliminary model that suggests there is no significant difference between normal conditions westbound and the change from normal conditions eastbound to congested conditions eastbound, the final classification tree is able to determine some significance. When comparing cluster 6 with clusters 8 and 9, there is a low level of significance, with p-values $<0.877$ (cluster 6 and cluster 8) and $<0.192$ (cluster 6 and 9). If total volumes for these two clusters are analyzed, the average total volume of cluster 6 is 146 and cluster 8 is 173 . This suggests that the two clusters produce the same air quality, with cluster 6 on average having 27 fewer trucks per five-minute interval. However, there are high levels of significance between cluster 6 and cluster 10,11 and 12. There is a low level of significance between cluster 7 and clusters 10 and 11. 


\subsubsection{Classification Tree Daytime Branch Three}

The next branch, on the classification tree Figure 5.9, occurs during the daytime hours when the westbound speed is greater or equal to $50 \mathrm{MPH}$ and westbound occupancies are greater than $18 \%$, this is a period where the Expressway is under some instability in the westbound direction while the eastbound direction maintains speeds greater than $50 \mathrm{MPH}$ and occupancies are less than or equal to $18 \%$. The final category shows the total class-nine truck counts and is divided into two different final branches: branch one has boundary conditions where total volumes are greater than 120 class-nine trucks and the second branch has boundary conditions where total volumes are less than or equal to 120 class-nine trucks. The 120 total class-nine boundary is selected to produce the greatest statistical separation between clusters 4 and 5 while providing some continuity between different clusters for direct comparison. Cluster 4 has an ambient $\mathrm{PM}_{2.5}$ flux of $33.5 \mathrm{ug} / \mathrm{m}^{2} *$ sec while cluster 5 has an ambient $\mathrm{PM}_{2.5}$ flux of 31.3 $\mathrm{ug} / \mathrm{m}^{2 *} \mathrm{sec}$. The ANOVA model indicates a statistical significance p-value 0.0567 . The statistical difference between the two clusters is explained by the impact of higher truck counts. The more trucks on the expressway, the higher the ambient $\mathrm{PM}_{2.5}$ flux.

Clusters 4 and 5 are compared with clusters 8 through 12. These cluster sets are different because clusters 4 and 5 have westbound occupancies greater than 18\% while clusters 8 through 12 have westbound occupancies less than or equal to 18\%. Direct ANOVA comparisons are made between clusters 4,8 , and 9 with p-values of 0.004 for cluster 8 and $<0.001$ for cluster 9 .

Figure 5.10 illustrates the difference between clusters 4, 8 and 9. Cluster 9 has a total truck count between 120 and 150 and cluster 8 has a total truck count greater than 150 . Comparing the three clusters, there is a 9.1\% decrease in air quality between cluster 8 and cluster 4, and a $14.7 \%$ decrease in air quality between cluster 9 and cluster 4 . The difference in ambient $\mathrm{PM}_{2.5}$ flux between clusters 4,8 , and 9 is explained by the only difference between the three clusters, the westbound occupancy. Cluster 8 on the average has a higher boundary truck limit, greater than 150 trucks per five minutes, compared to either the 120 or greater for cluster four or the range from 120 to 150 trucks for cluster 9. From an operational standpoint, the biggest change in ambient $\mathrm{PM}_{2.5}$ flux, under these operational conditions, stems from the importance of maintaining westbound occupancies lower than $18 \%$. With the same truck volumes traveling along the Borman Expressway, the increase in westbound occupancy to levels greater than 18\% decreases the ambient air quality by $9.1 \%$ or higher. 


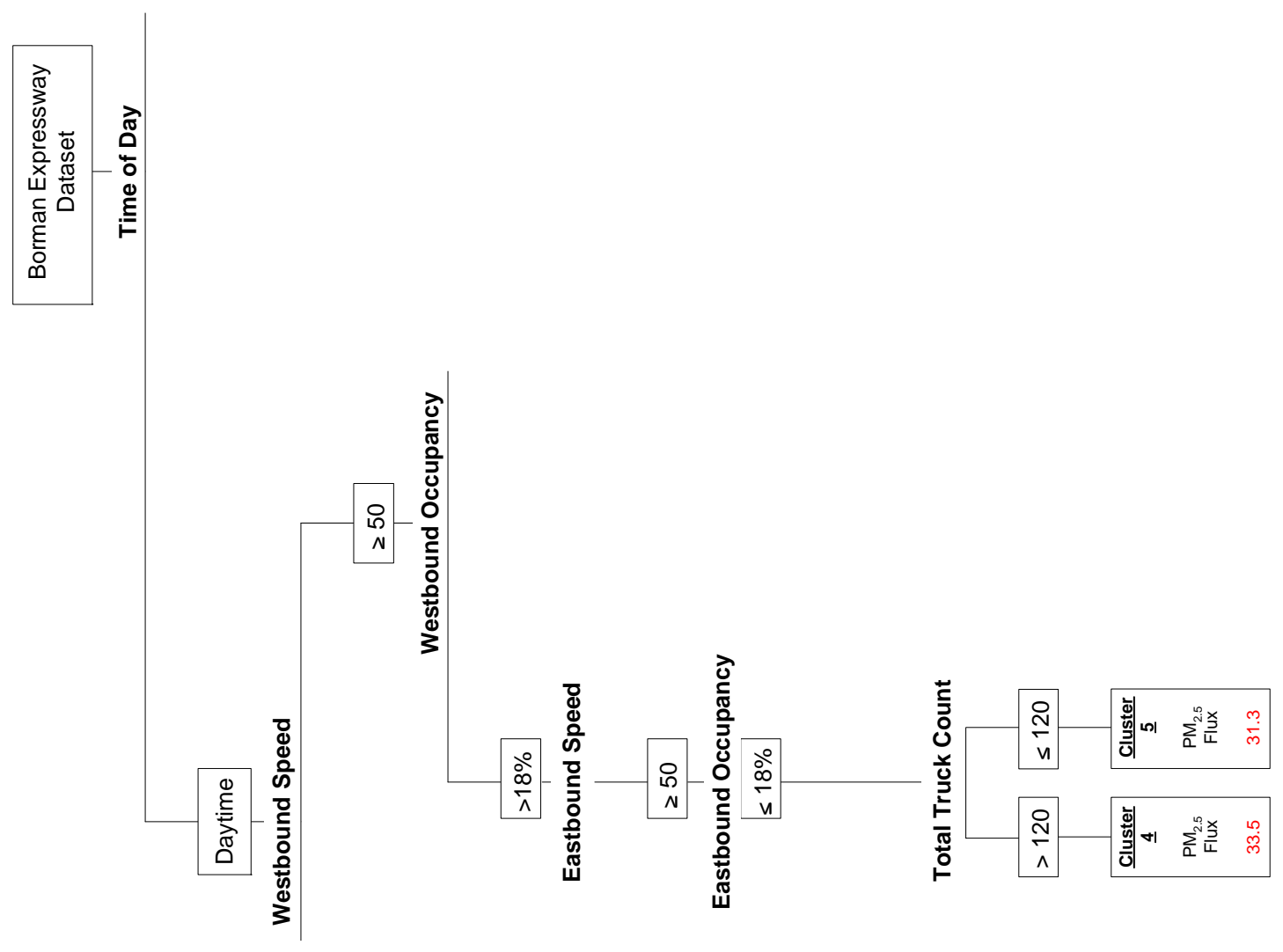

Figure 5.9: Daytime Classification Tree for Increased Instability in the Westbound Direction 
$\mathrm{PM}_{2.5}$ Flux vs. Decision Tree Westbound Comparison

Borman Expressway

Daytime Operational Hours

6:00 AM to 8:00 PM

January 2002 to June 2002

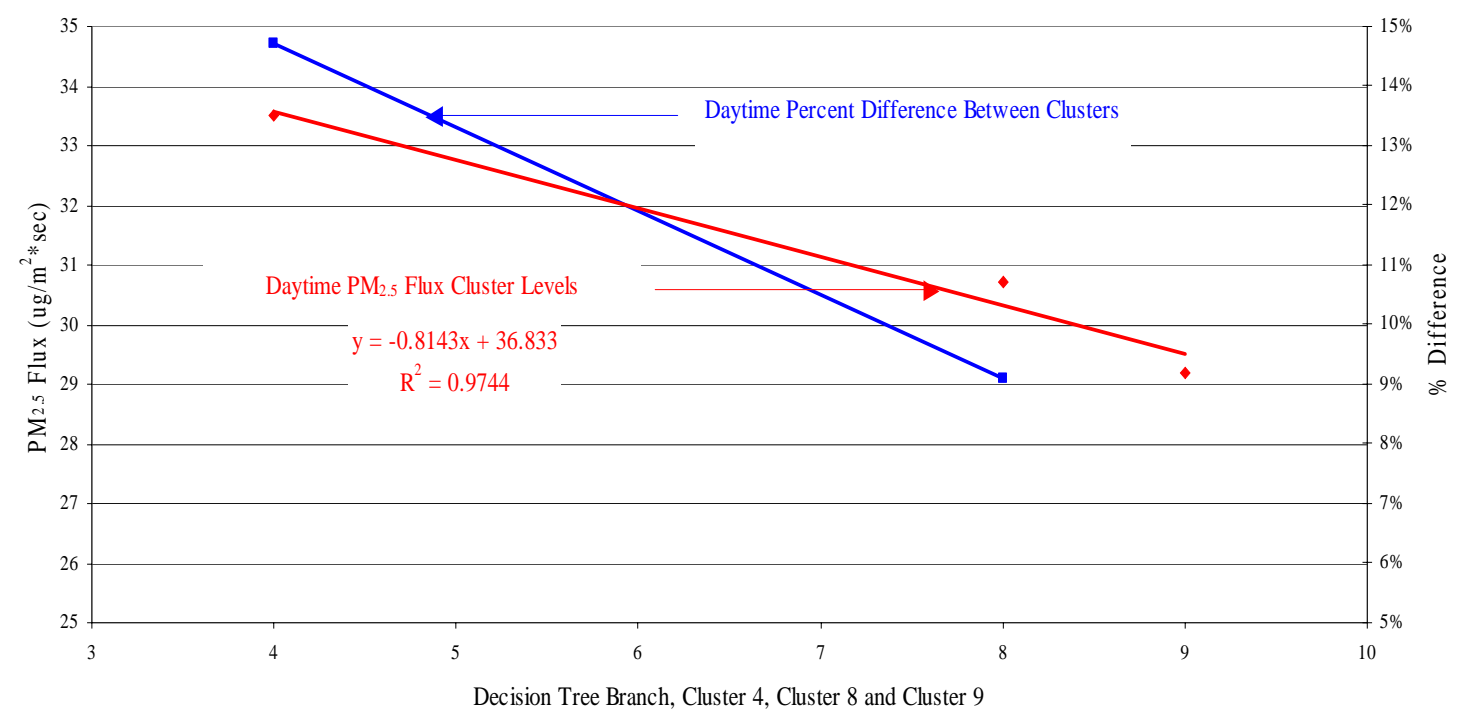

Figure 5.10: Daytime Classification Tree Westbound Comparison

The second comparison between cluster 5 and clusters 10 through 12 shows similar trends, as seen between clusters 4, 8 and 9 . Cluster 5 remains at the highest level of ambient $\mathrm{PM}_{2.5}$ flux, with a level of significance p-value $<0.0001$ in all comparisons. This suggests the same factors are involved between the increase in westbound occupancy from less than $18 \%$ to greater than $18 \%$ even under lower traffic volumes ( $<120$ trucks per five-minute intervals). For both sets of traffic volumes, it is extremely important to manage the westbound occupancies. The impact from higher occupancies, regardless of total traffic counts, decreases the ambient $\mathrm{PM}_{2.5}$ flux air quality.

\subsubsection{Classification Tree Daytime Branch Four}

The final set of branches, Figure 5.11, describes the worst-case scenarios along the Borman Expressway. The operational hours occur during the daytime, when the westbound speeds are less than or equal to $45 \mathrm{MPH}$ with westbound occupancies greater than $10 \%$. The eastbound is divided into two different branches, speeds greater than or equal to $50 \mathrm{MPH}$ and speeds less than or equal to 45MPH. Clusters 2 and 3 are divided by the eastbound truck counts with boundary conditions of less than or equal to 59 trucks and greater than 59 trucks. A 59 
average class-nine vehicle volume is selected because it provides the greatest statistical significance, as well as similar sample sizes and is the average value under these conditions. The total vehicle volume is not selected for the same reasons as clusters 6 and 7 . Clusters 2 and 3 occur while the westbound lanes of operation are under heavy congestion. During these periods the volumes can change dramatically and are highly unstable. Due to the variability in the westbound conditions, the average westbound volume is not used. As shown earlier, the overall ambient air quality is the product of both the eastbound and the westbound conditions. In this case, the westbound conditions are under heavy congestion and the eastbound conditions remain under normal conditions. The branch between the two levels of truck volumes in the eastbound direction account for periods with higher levels of truck volumes, greater than 59 trucks per fiveminute interval, and lower levels of truck volumes, less than or equal to 59 trucks per five-minute intervals.

In both cases, there is a significant statistical difference, p-value of 0.0086 , between clusters 2 and 3. Cluster 2 has a $\mathrm{PM}_{2.5}$ flux value of 38.8 (ug/ $\left.\mathrm{m}^{2} * \mathrm{sec}\right)$, while cluster 3 has a value of 34.8 (ug/ $\left./ \mathrm{m}^{2 *} \mathrm{sec}\right)$. Cluster 2 represents high volumes of class-nine trucks in the eastbound direction and breakdown levels in the westbound direction. There is a decrease in air quality of $11.5 \%$ with the change in eastbound truck volumes.

The final branch on the classification tree is the worst-case scenario. Cluster 1 includes breakdown periods for the eastbound and the westbound traffic. In this case the Expressway is highly inefficient, with low speeds and high engine loadings that contribute to the poorest ambient air quality (Metz et al. 1997, Yanowitz et al. 2002). The $\mathrm{PM}_{2.5}$ flux value is 40.9 (ug/ $\mathrm{m}^{2} * \mathrm{sec}$ ), an increase of $87.6 \%$ as compared to cluster 12 , the best-case daytime scenario. With the exception of cluster 2 , p-value of 0.24 , cluster 1 is significantly different than all the other clusters on the classification tree with p-values less than 0.0006. In terms of Expressway management, air quality is highly dependent on the westbound lanes. When the westbound lanes are experiencing heavy congestion, the air quality surrounding the Borman Expressway decreases.

As shown earlier, Figure 5.3, combines all the daytime and nighttime branches of the classification tree into one figure, and as previously explained, accounts for $97.7 \%$ of all the different conditions along the expressway. 


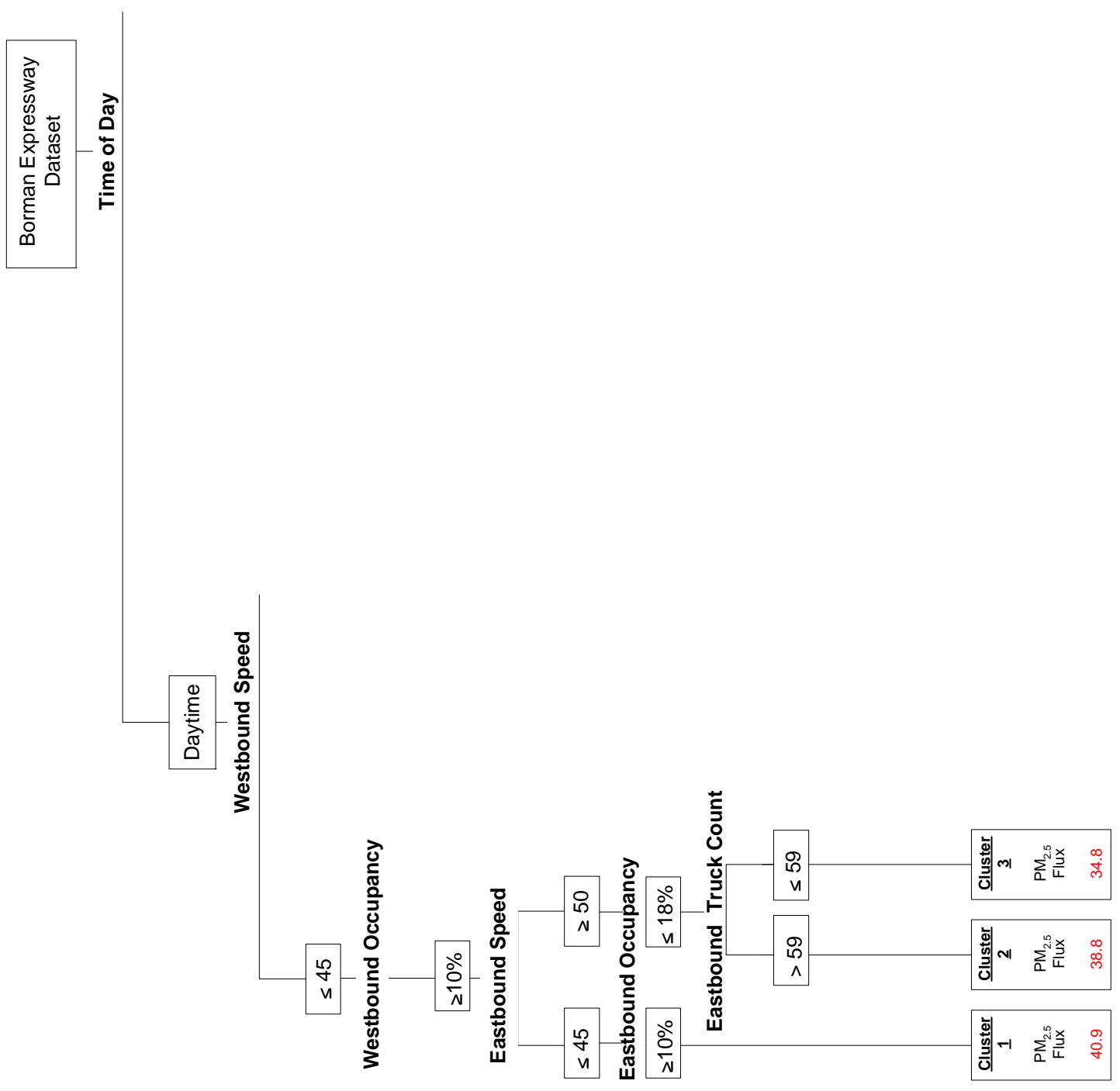

Figure 5.11: Daytime Classification Tree Westbound Congestion

Table 5.6, is a summary of the ANOVA model that is used to statistically identify the different cluster levels that are used for the classification tree shown in Figure 5.3, and the other statistical analyses from this chapter. The numerical values represented in the ANOVA model are the same model values used for the different cluster levels. 
Table 5.6: ANOVA Output for the Different Cluster Conditions

\author{
The SAS System \\ The GLM Procedure \\ Class Level Information
}

$\begin{array}{ccc}\text { Class } & \text { Levels } & \text { Values } \\ \text { Cluster } & 16 & 12345678910111213141516\end{array}$

Least Squares Means for effect cluster

$\operatorname{Pr}>|t|$ for H0: LSMean(i)=LSMean(j)

Dependent Variable: PM2.5flux

$\begin{array}{ccccccccc}\mathrm{i} / \mathrm{j} & 1 & 2 & 3 & 4 & 5 & 6 & 7 & 8 \\ & & & & & & & & \\ 1 & & 0.2491 & 0.0006 & <.0001 & <.0001 & <.0001 & <.0001 & <.0001 \\ 2 & 0.2491 & & 0.0086 & <.0001 & <.0001 & <.0001 & <.0001 & <.0001 \\ 3 & 0.0006 & 0.0086 & & 0.3246 & 0.0146 & 0.0247 & <.0001 & 0.0004 \\ 4 & <.0001 & <.0001 & 0.3246 & & 0.0567 & 0.0859 & <.0001 & 0.0004 \\ 5 & <.0001 & <.0001 & 0.0146 & 0.0567 & & 0.8536 & 0.0070 & 0.6191 \\ 6 & <.0001 & <.0001 & 0.0247 & 0.0859 & 0.8536 & & 0.0284 & 0.8770 \\ 7 & <.0001 & <.0001 & <.0001 & <.0001 & 0.0070 & 0.0284 & & 0.0045 \\ 8 & <.0001 & <.0001 & 0.0004 & 0.0004 & 0.6191 & 0.8770 & 0.0045 & \\ 9 & <.0001 & <.0001 & <.0001 & <.0001 & 0.0436 & 0.1920 & 0.0958 & 0.0038 \\ 10 & <.0001 & <.0001 & <.0001 & <.0001 & <.0001 & 0.0035 & 0.9946 & <.0001 \\ 11 & <.0001 & <.0001 & <.0001 & <.0001 & <.0001 & 0.0001 & 0.3635 & <.0001 \\ 12 & <.0001 & <.0001 & <.0001 & <.0001 & <.0001 & <.0001 & 0.0170 & <.0001 \\ 13 & <.0001 & <.0001 & <.0001 & <.0001 & <.0001 & <.0001 & 0.1904 & <.0001 \\ 14 & <.0001 & <.0001 & <.0001 & <.0001 & <.0001 & <.0001 & 0.0191 & <.0001 \\ 15 & <.0001 & <.0001 & <.0001 & <.0001 & <.0001 & <.0001 & <.0001 & <.0001 \\ 16 & <.0001 & <.0001 & <.0001 & <.0001 & <.0001 & <.0001 & <.0001 & <.0001\end{array}$

The SAS System

Least Squares Means for effect cluster $\operatorname{Pr}>|t|$ for H0: LSMean(i)=LSMean(j)

Dependent Variable: pmflux

\begin{tabular}{|c|c|c|c|c|c|c|c|c|}
\hline & 9 & 10 & 11 & 12 & 13 & 14 & 15 & 16 \\
\hline 1 & $<.0001$ & $<.0001$ & $<.0001$ & $<.0001$ & $<.0001$ & $<.0001$ & $<.0001$ & $<.0001$ \\
\hline 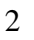 & $<.0001$ & $<.0001$ & $<.0001$ & $<.0001$ & $<.0001$ & $<.0001$ & $<.0001$ & $<.0001$ \\
\hline 3 & $<.0001$ & $<.0001$ & $<.0001$ & $<.0001$ & $<.0001$ & $<.0001$ & $<.0001$ & $<.0001$ \\
\hline 4 & $<.0001$ & $<.0001$ & $<.0001$ & $<.0001$ & $<.0001$ & $<.0001$ & $<.0001$ & $<.0001$ \\
\hline 5 & 0.0436 & $<.0001$ & $<.0001$ & $<.0001$ & $<.0001$ & $<.0001$ & $<.0001$ & $<.0001$ \\
\hline 6 & 0.1920 & 0.0035 & 0.0001 & $<.0001$ & $<.0001$ & $<.0001$ & $<.0001$ & $<.0001$ \\
\hline 7 & 0.0958 & 0.9946 & 0.3635 & 0.0170 & 0.1904 & 0.0191 & $<.0001$ & $<.0001$ \\
\hline 8 & 0.0038 & $<.0001$ & $<.0001$ & $<.0001$ & $<.0001$ & $<.0001$ & $<.0001$ & $<.0001$ \\
\hline 9 & & 0.0001 & $<.0001$ & $<.0001$ & $<.0001$ & $<.0001$ & $<.0001$ & $<.0001$ \\
\hline 10 & 0.0001 & & 0.0314 & 0.0035 & 0.0055 & $<.0001$ & $<.0001$ & $<.0001$ \\
\hline 11 & $<.0001$ & 0.0314 & & 0.0244 & 0.3072 & 0.0001 & $<.0001$ & $<.0001$ \\
\hline 12 & $<.0001$ & 0.0035 & 0.0244 & & 0.0615 & 0.2748 & 0.4760 & 0.0036 \\
\hline 13 & $<.0001$ & 0.0055 & 0.3072 & 0.0615 & & 0.0233 & $<.0001$ & $<.0001$ \\
\hline 14 & $<.0001$ & $<.0001$ & 0.0001 & 0.2748 & 0.0233 & & $<.0001$ & $<.0001$ \\
\hline 15 & $<.0001$ & $<.0001$ & $<.0001$ & 0.4760 & $<.0001$ & $<.0001$ & & $<.0001$ \\
\hline 16 & $<.0001$ & $<.0001$ & $<.0001$ & 0.0036 & $<.0001$ & $<.0001$ & $<.0001$ & \\
\hline
\end{tabular}




\section{CLUSTER-FREQUANCY OF OCCURRENCE ON THE BORMAN EXPRESSWAY}

\subsection{Frequency of Occurrence for the Sixteen Clusters}

\subsubsection{Daytime Frequency of Occurrence for the Twelve Clusters}

Figure 6.1 is a pie graph of the frequency of occurrence for the 12 different daytime clusters. Clusters 8 through 12 comprise the majority, or 83\%, of the traffic conditions, which include speeds greater than or equal to $50 \mathrm{MPH}$ and occupancies less than or equal to $18 \%$. The difference between clusters 8 through 12 consists of the change in truck volumes, with cluster 8 having the largest truck volumes ( $>150)$ and cluster 12 having the smallest $(<30)$.

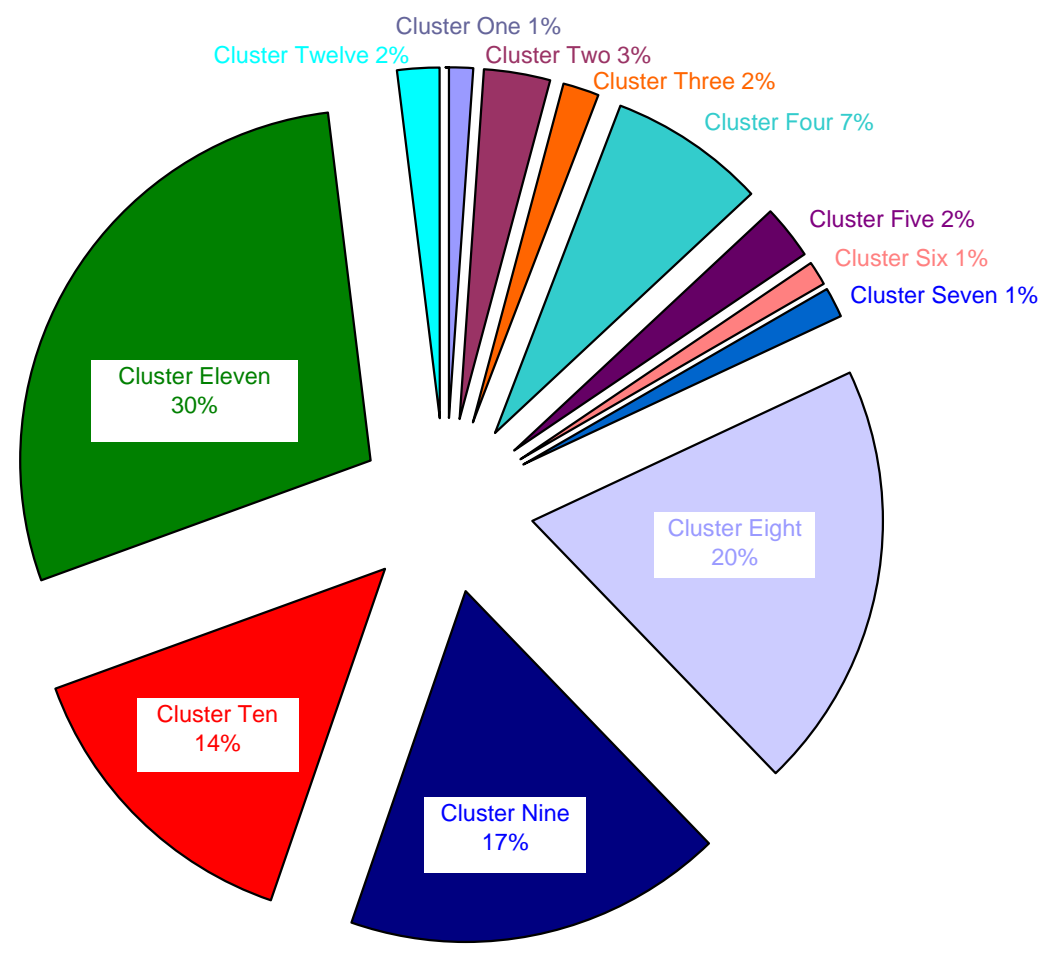

Figure 6.1: Daytime Frequency of Occurrence for the Different Clusters

The three worst-case scenarios are clusters 1, 2 and 3, which represent $5 \%$ of total traffic conditions. This may seem like a small problem, but on average the first three clusters exhibit $33 \%$ worse air quality when directly compared with clusters 8 through 12 . There is a $46 \%$ change in ambient air quality between the worst-case cluster $1\left(40.9 \mathrm{ug} / \mathrm{m}^{2 *} \mathrm{sec}\right)$ and the best-case cluster 
$12\left(21.6 \mathrm{ug} / \mathrm{m}^{2} * \mathrm{sec}\right)$. From a traffic management strategy, clusters 10 through 12 are the most desired, with normal operating conditions and relatively stable traffic flow.

In terms of measuring the impact from ITS, the frequency of occurrence during the daytime hours for clusters 1 through 3 is $1 \%$, 3\%, and 2\% respectively. With the improvement in traffic flow as a direct results of ITS strategies, clusters 1 through 3 decrease their frequency of occurrence, while the clusters 9 through 12 increase their frequency of occurrence resulting in better local ambient air quality.

\subsubsection{Nighttime Frequency of Occurrence for the Different Clusters}

The nighttime activity on the Borman Expressway as seen in Chapter 4 is more stable than the daytime so the classification tree model in Chapter 5 arranges the nighttime traffic into four different clusters that include speeds greater or equal to $50 \mathrm{MPH}$ and occupancies less than or equal to $18 \%$. The difference between the four nighttime clusters results from the change in class-nine truck volumes. Figure 6.2 depicts the frequency of occurrence for the different nighttime clusters. Clusters 14 and 15 have the highest frequency of occurrence with $69 \%$ of the total traffic activity, which suggests that the majority of the class-nine truck volume during the nighttime hours is between 30 and 120 combined class-nine trucks per five-minutes.
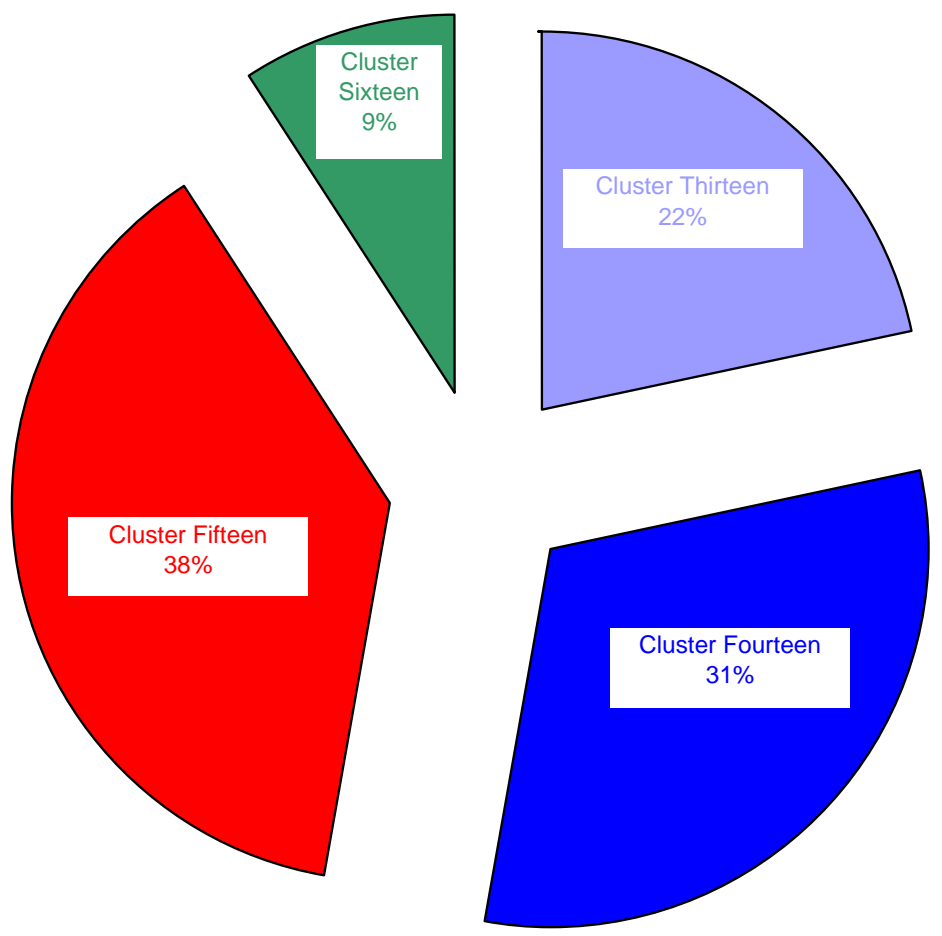

Figure 6.2: Nighttime Frequency of Occurrence for Different Clusters 
Cluster 16, with the best ambient air quality, occurs $9 \%$ of the time, an increase of $7 \%$ over the daytime hours. Again this illustrates the more stable nature of nighttime operations. 


\section{SUMMARY AND CONCLUSIONS}

The Borman Expressway project was concerned with monitoring and studying the impact of mobile sources of the expressway on the local air quality. Although being surrounded by major stationary industrial sources, earlier phases of this project has found that the Borman Expressway supplied on average $77 \%$ of the $\mathrm{CO}$ and $72 \%$ of the $\mathrm{PM}_{2.5}$ as determined by modeling and regression analysis of traffic and environment data (SPR-2338). More over, it has been shown that the Carbon monoxide and $\mathrm{PM}_{2.5}$ concentrations and flux can increase by a factor of 2 to 3 over pre-incident / pre - congestion conditions during accident / congestion events (SPR-2338). This shows that any means that can prevent or reduce oversaturated traffic conditions should substantially reduce the presence of pollution in the local environment.

Therefore, a descriptive model was developed during this phase of the project in the form of a classification tree, which characterizes the impact of different Borman-traffic levels on the local ambient air quality. This tree contains 16 different traffic clusters and shows a $46 \%$ improvement in local air quality from the worst-case congestion levels along the Borman Expressway to the best-case ones. The classification tree was based on the following factors:

1. Time of Day

2. Westbound Class-Nine Average Speed

3. Westbound Occupancy

4. Eastbound Class-Nine Average Speed

5. Eastbound Occupancy

6. Westbound Class-Nine Truck Count

7. Eastbound Class-Nine Truck Count

8. The Total Eastbound and Westbound Truck Counts

The developed descriptive model has an advantage over other simulation models since it is based on robust measured data from the Borman Expressway, hence; provide more realistic output and consequently more realistic analysis 


\section{LIST OF REFERENCES}

Birst, Shawn., Smadi, Ayman., “An Application of ITS for Incident Management in SecondTier Cities: A Fargo, ND Case Study.” Mid-Content Transportation Symposium 2000

Proceeding, 2000.

Burnett, Richard T. Cakmak, Sabit. Raizenne, Mark E. Stieb, David. Vincent, Renaud. Krewski, Daniel. Brook, Jeffrey R. Philips, Owen. and Ozkaynak, Haluk., “Association Between Ambient Carbon Monoxide Levels and Daily Mortality in Toronto, Canada”, Journal of Air and Waste Management Association, vol. 48 n 8, pp. 689-700, August 1998.

Chan, Chang-Chuan, Halûk Özkayank, John D. Spengler, and Linda Sheldon, "Driver Exposure to Volatile Organic Compounds, $\mathrm{CO}$, Ozon, and $\mathrm{NO}_{2}$ Under Different Driving Conditions”, Environ. Sci. Technol., vol. 25, No. 5, 1991, pp. 964-972.

Chquetto, Sergio, and Roger Mackett "Modelling the Effects of Transport Policies on Air Pollution”, The Science of The Total Environment, vol. 169, pp. 265-271, 1995.

Coifman, Benjamin, "Estimating Travel Times and Vehicle Trajectories on Freeways Using Dual Loop Detectors", under review, submitted for publication in Transportation ResearchB.

Coombs, W M., “Occupational Medicine Effects of Carbon Monoxide”, Journal of Mine Ventilation Society of South Africa, vol. 51 n 4, pp. 140-141, Oct-Dec 1998.

Davis, Mackenzie L., and David A. Cornwell, Introduction to Environmental Engineering, $3^{\text {rd }}$ edition, p. 471 (WCB/McGraw-Hill, New York, 1998).

Degobert, Paul, Automobiles and Pollution, (Society of Automotive Engineers, PA, 1995).

Klemm, Rebecca., Mason, Robert., Heilig, Charles., Neas, Lucas., Dockery, Douglas., "Is Daily Mortality Associated Specifically With Fine Particles? Data Reconstruction and Replication of Analyses.” Journal of Air and Waste Management Association, Vol. 50, No. 7, pp. 1215-1222, Jul. 2000

Latoski, SP., Pal, R., Sinha, KC., "Cost-Effectiveness Evaluation of Hoosier Helper Freeway Service Patrol.” Journal of Transportation Engineering, Vol. 125, No.5, pp. 429438, Sept. 1999.

Lomax, Tim., Schrank, David., “Urban Mobility Study-1997.” Texas Transportation Institute, Texas A\&M, 1999.

Mage, David., Guntis Ozolins, Peter Peterson, Anthony Webster, Rudi Orthofer, Veerle Vandeweerd, and Michael Gwynne, "Urban Air Pollution in Megacities of the World”, Atmospheric Environment, vol. 30, n 5, pp. 681-686, 1991.

Mayer, Helmut, “Air Pollution in Cities”, Atmospheric Environment, vol. 33, pp. 40294037, 1999. 
Metz, N., Schlichter, H., Schellenberg., "Positive Effects of a Traffic Control System on Fuel Consumption and Exhaust Emission on the German A9 Autobahn.” International Journal of Vehicle Design, Vol. 18, No. 3-4, pp. 354-367, 1997.

Niemeier, D., Utts, J., Fay, L., “Cluster Analysis for Optimial Sampling of Traffic Data: Air Quality Example.” Journal of Transportation Engineering, Vol. 128, No. 1, pp. 97-101, Feb. 2002.

Noll, Kenneth E., and Terry L. Miller, Air Monitoring Survey Design, p. 67 (Ann Arbor Science Publishers Inc., Michigan, 1977).

Seinfeld, John H., Air Pollution, Physical and Chemical fundamentals, pp. 71 (McGrawHill, New York, 1975).

SPR-2338, Joint Transportation Research Program (JTRP), File No. 9-10-51, Project No. C36-97ZZ, (FHWA/A/IN/JTRP-2002/5), September 2002.

Vedal, Sverrs., “Ambient Particle Health: Lines that Divide.” Journal of The Air and Waste Management Association, Vol. 47, No. 5, pp. 551-581, 1997.

Wark, Kenneth., Cecil F. Warner, and Wayne T. Davis, Air Pollution, its Origin and Control, $3^{\text {rd }}$ edition, pp. 70, 189 (Addison-Wesley, California, 1998).

Wofsy, S. C., McConnell, J. C., and McElroy, M. B., "Atmospheric $\mathrm{CH}_{4}$, CO, and $\mathrm{CO}_{2}$ ”, Journal of Geophysical Research, vol. 67, pp. 4477-4493, 1972. 


\section{APPENDIX}

Due to the constant construction and improvements on the Borman Expressway, continuous traffic data collection that matches the continuity of the environmental data is hard to obtain. Such continuous traffic data sets are necessary for long period analyses as well as for upto-date data analysis. Therefore, a side-fire WAVETRONIX ${ }^{\mathrm{TM}}$ traffic SMART-SENSOR $^{\circledR}$ has been obtained on (DATE) to be installed at the PU-Hessville Site.

The capabilities of the sensor have been tested on a local road in West Lafayette, IN for a period of 3 weeks in May 2004. It has shown the ability of providing both event and interval traffic data continuously. This testing period allowed for the unit calibration and the debugging of the operating software.

Interval data log files provide time/date stamped traffic volume, occupancy, and timemean-speeds as shown on figures A.1 through A.3.

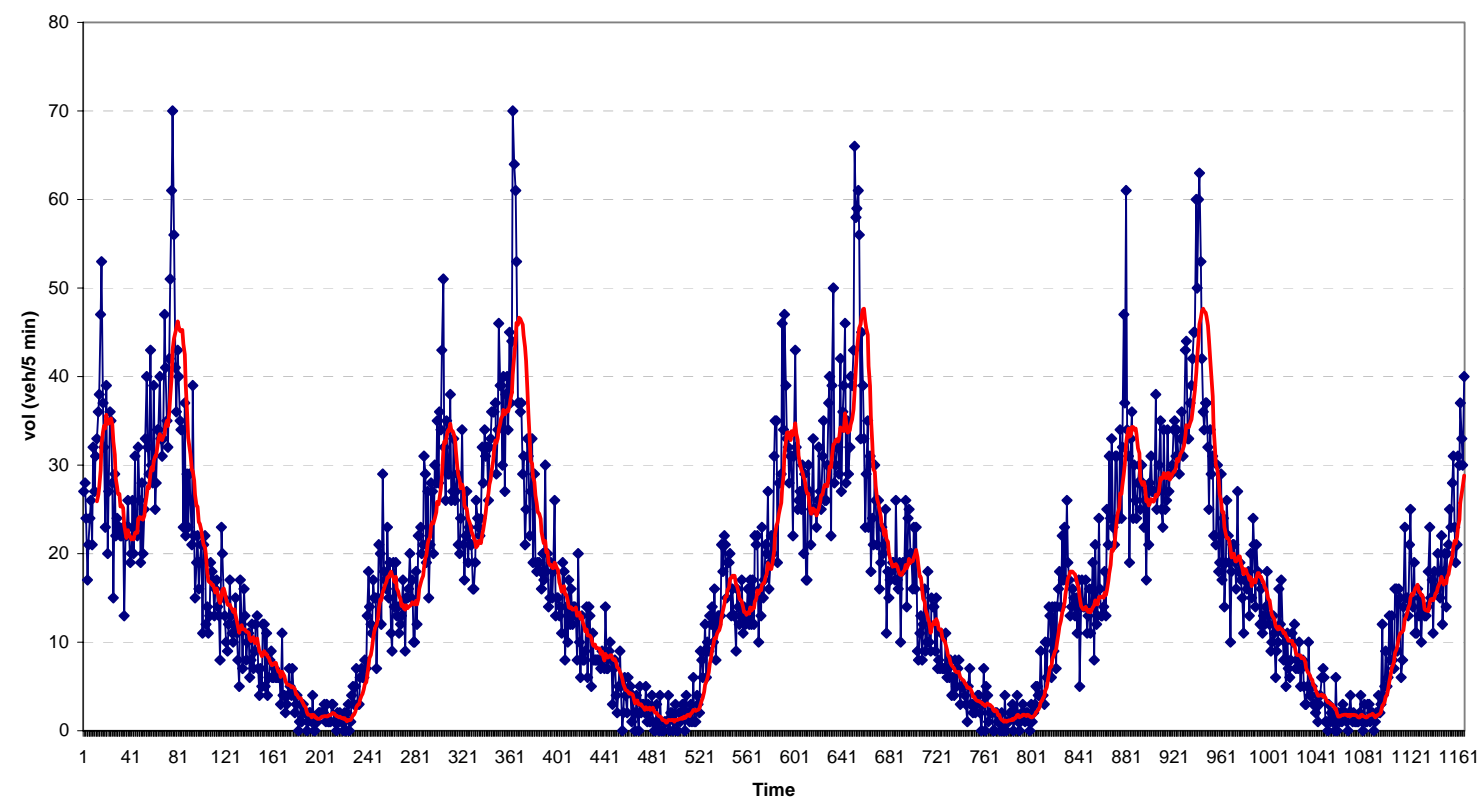

Figure A.1: A sample of 5-min averaged traffic volume on Northwestern Ave, West Lafayette, IN. May 24 to May 28, 2004. 


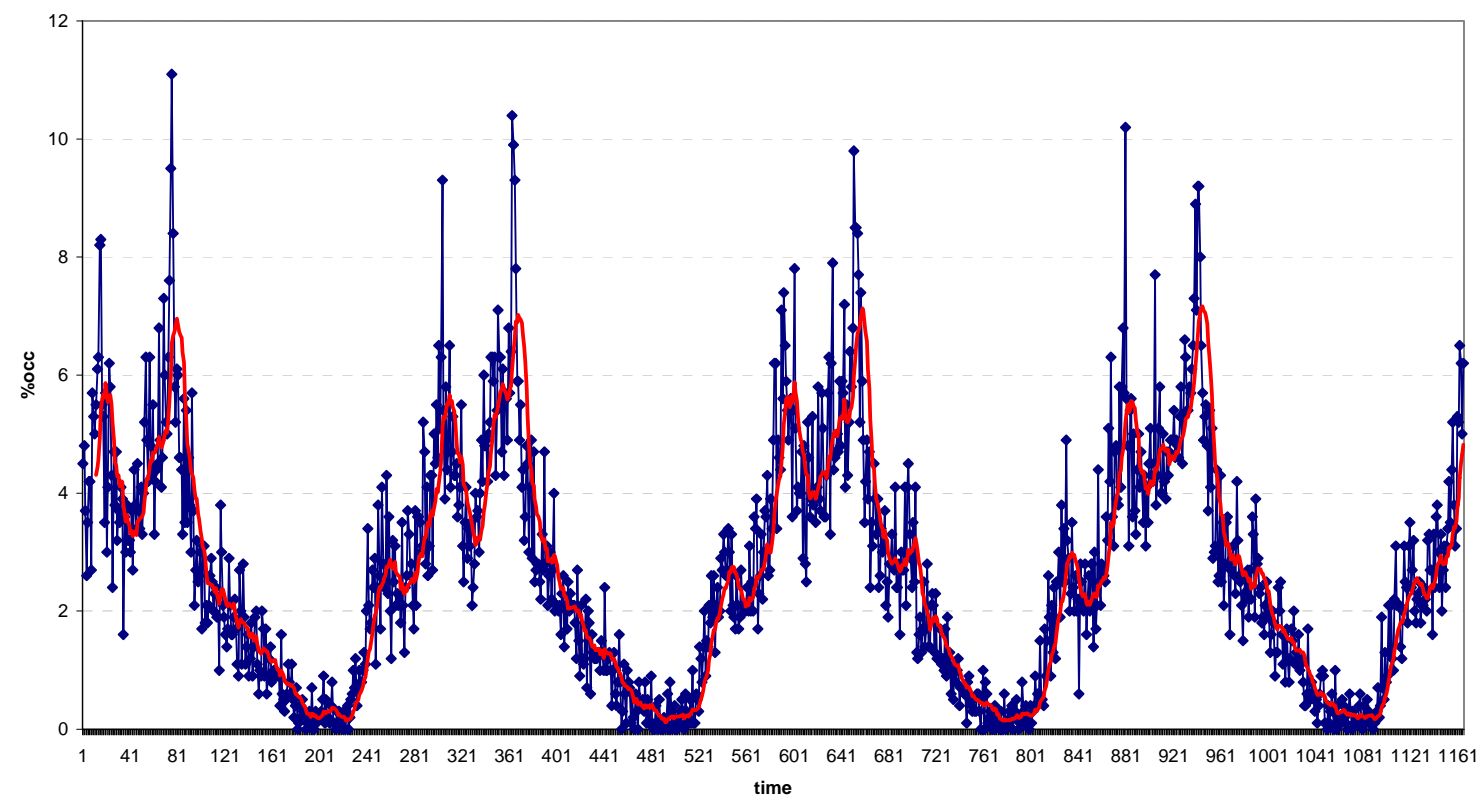

Figure A.2: A sample of 5-min averaged traffic occupancy on Northwestern Ave, West Lafayette, IN. May 24 to May 28, 2004.

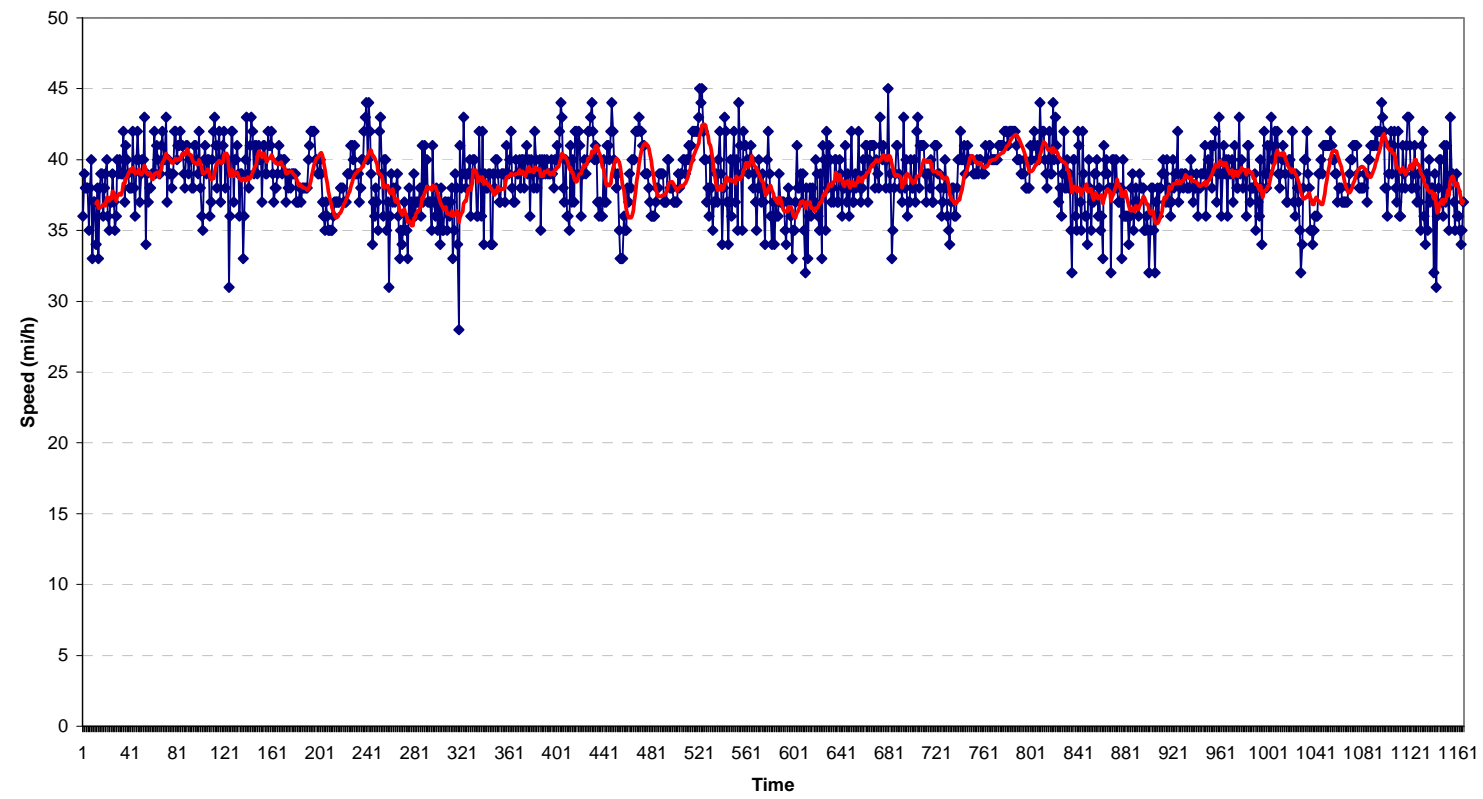

Figure A.3: A sample of 5-min averaged traffic time-mean-speed on Northwestern Ave, West Lafayette, IN. May 24 to May 28, 2004. 
Event data log files on the other hand, provide time stamped vehicle speeds as well as classification. This type of data set is useful in providing travel time estimates on a road segment using Coifman's one dual-loop algorithm (Coifman, under review). Figure A.4 shows the estimated travel time for Northwestern Ave., West Lafayette, IN for a downstream road segment of 0.5 mile in length, in a 35 MPH speed zone. Space-mean-speeds calculated from the estimated travel times are used in comparison to the measured speeds to verify the accuracy of the algorithm as shown in figure A.5.

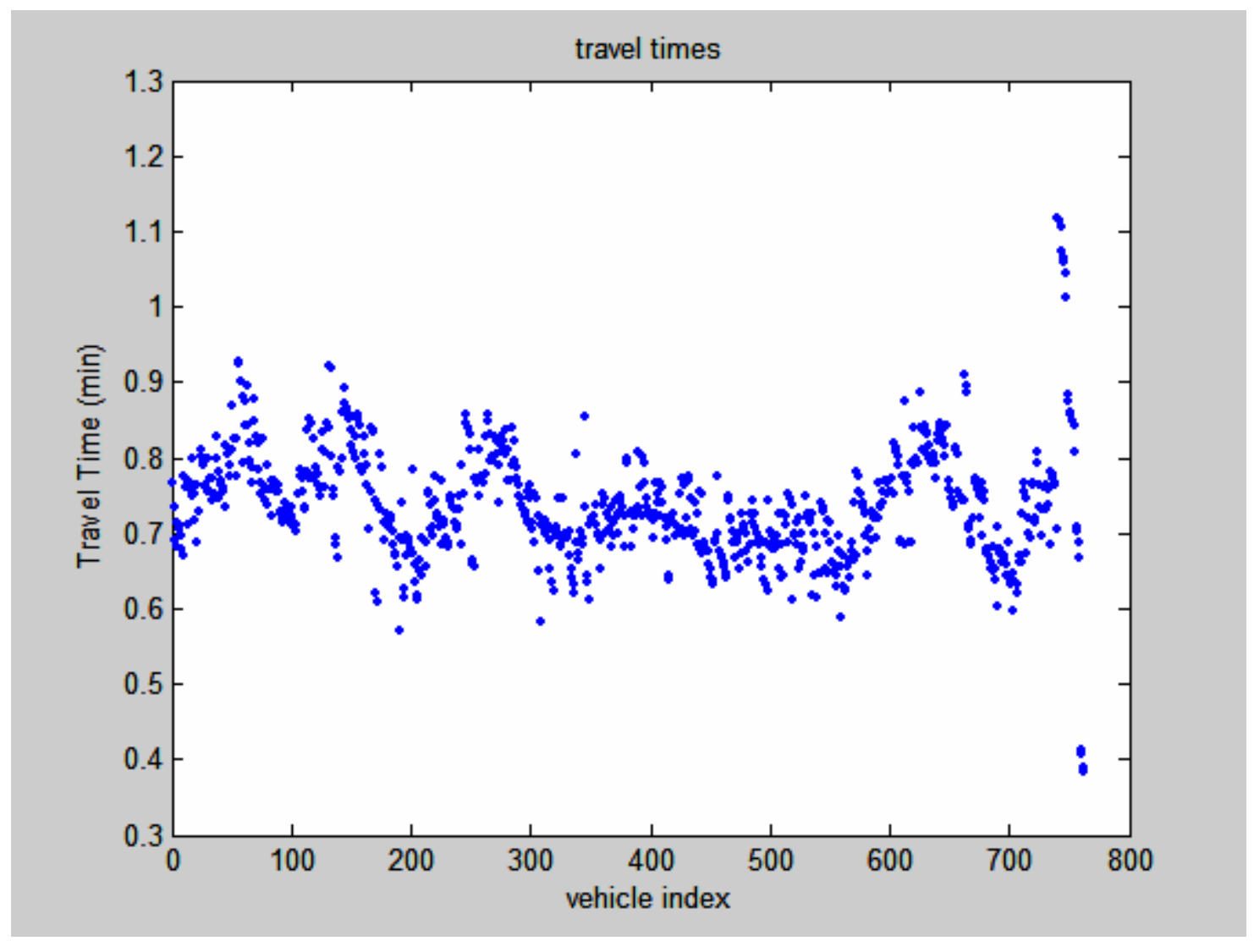

Figure A.4: Estimated travel times on Northwestern Ave, West Lafayette, IN, for a lane segment of 0.5 miles. May 13, 2004, from 1:40 PM to 5:20 PM.

As shown in figure A.4, travel times exhibit periodic behavior similar to the ones shown for occupancy and volumes, indicating the fact which is the increase of travel times during heavy traffic conditions. Although figure A.5 has shown a good match between measured speeds (dotted line) and calculated speeds (solid line), more analysis and further verification using both 
calculated and observed travel times are needed and are still under way before installing the sensor at the PU-Hessville Site on the Borman Expressway.

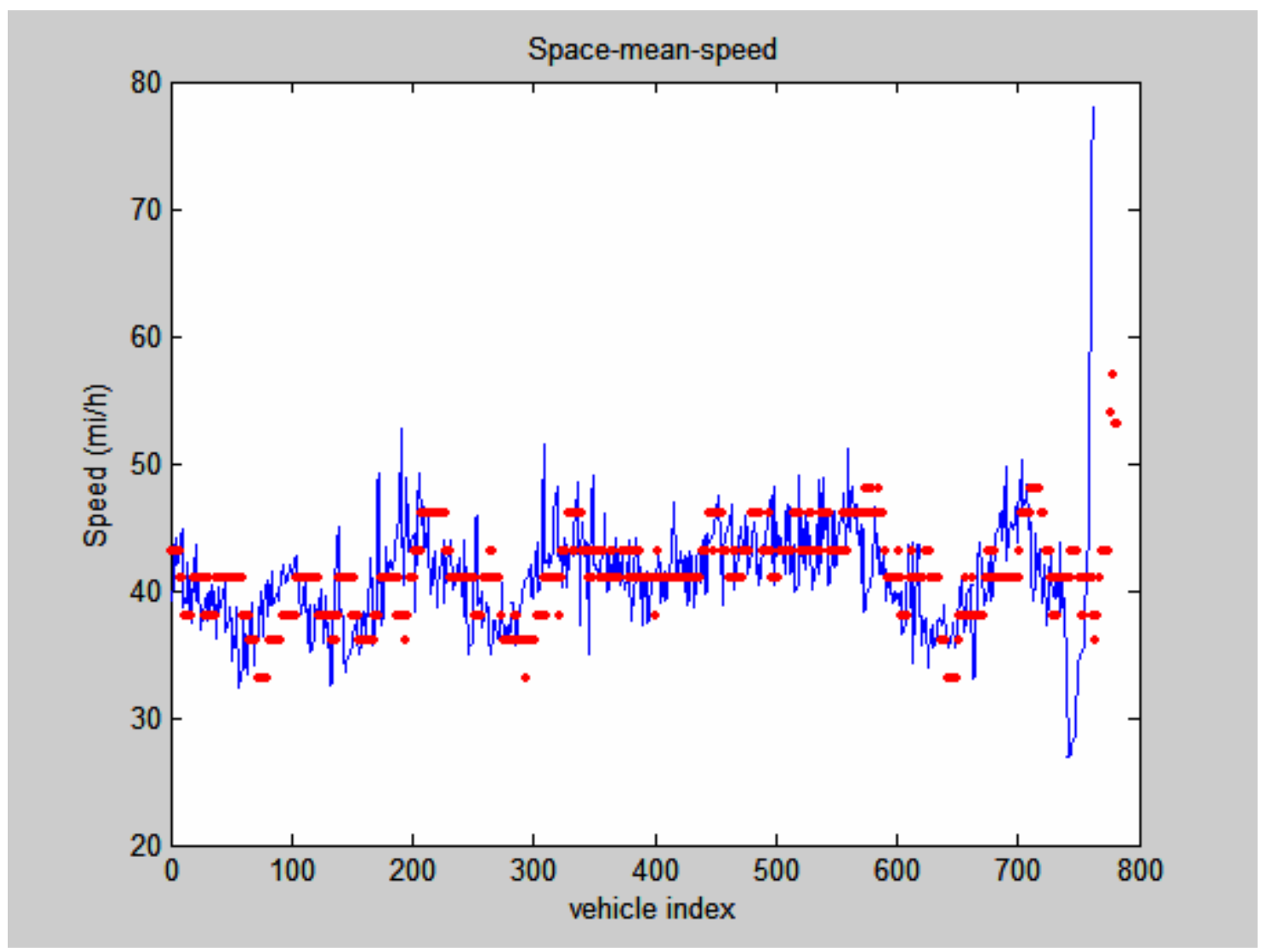

Figure A.5: Estimated space-mean-speeds (solid line), and time-mean-speeds (dotted line) on Northwestern Ave, West Lafayette, IN, for a lane segment of 0.5 miles. May 13, 2004, from 1:40 PM to 5:20 PM. 